\title{
On the cohomology rings of real flag manifolds: Schubert cycles
}

\author{
Ákos K. Matszangosz ${ }^{1}$ (D) \\ Received: 2 November 2020 / Revised: 23 April 2021 / Accepted: 2 July 2021 / \\ Published online: 31 July 2021 \\ (c) The Author(s) 2021
}

\begin{abstract}
We give an algorithm to compute the integer cohomology groups of any real partial flag manifold, by computing the incidence coefficients of the Schubert cells. For even flag manifolds we determine the integer cohomology groups, by proving that any torsion class has order 2 (generalizing a result of Ehresmann). We conjecture that this holds for any real flag manifold. We obtain results concerning which Schubert varieties represent integer cohomology classes, their structure constants and how to express them in terms of characteristic classes. For even flag manifolds and Grassmannians we also describe Schubert calculus. The Schubert calculus can be used to obtain lower bounds for certain real enumerative geometry problems (Schubert problems).
\end{abstract}

Mathematics Subject Classification 14M15 - 57T15 primary; 14P25 - 55N91 . $57 \mathrm{~N} 80 \cdot 57 \mathrm{R} 95$ secondary

\section{Contents}

1 Introduction . . . . . . . . . . . . . . . . . . . . . . . . 1538

1.1 Additive structure . . . . . . . . . . . . . . . . . . . . . . . 1538

1.1 .1 Incidence coefficients . . . . . . . . . . . . . . . . . . . . . . 1538

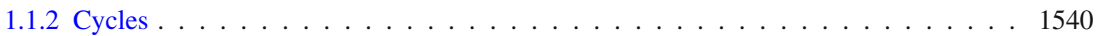

1.2 Structure constants . . . . . . . . . . . . . . . . . . . . . . . 1540

1.3 Characteristic classes . . . . . . . . . . . . . . . . . . . . . . . . 1540

1.4 Summary of the results on $H^{*}\left(\mathrm{Fl}_{\mathcal{D}}^{\mathbb{R}} ; \mathbb{Q}\right) \ldots \ldots \ldots \ldots \ldots \ldots \ldots \ldots 1 \ldots \ldots \ldots$

1.5 Integer coefficients . . . . . . . . . . . . . . . . . . . . . . . . . . 1542

Communicated by Jean-Yves Welschinger.

This research was partially supported by the Hungarian National Research, Development and Innovation Office, NKFIH K 119934.

$凶$ Ákos K. Matszangosz

matszangosz.akos@gmail.com

1 Alfréd Rényi Institute of Mathematics, Budapest, Hungary 
1.6 Applications - real enumerative geometry . . . . . . . . . . . . . . . . . . . . . . . . 1542

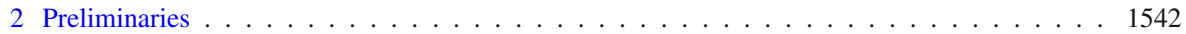

2.1 Vassiliev complex - incidence coefficients . . . . . . . . . . . . . . . . . . . 1543

2.2 Geometry of flag manifolds . . . . . . . . . . . . . . . . . . . . . . . . . . 1544

2.2.1 Schubert varieties, orbit structure . . . . . . . . . . . . . . . . . . . . . . . . . . . . . . . . . . . . . . . . . . . . . . . . . .

2.2.2 Tangent bundle of $\mathrm{Fl}_{\mathcal{D}}\left(\mathbb{R}^{N}\right) \ldots \ldots \ldots \ldots \ldots \ldots \ldots \ldots$

2.2 .3 Direct sum maps . . . . . . . . . . . . . . . . . . . . . . . . . . . . 1546

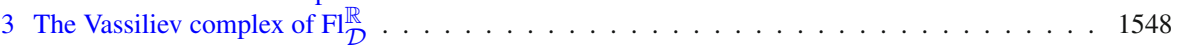

3.1 Coorientation of the strata . . . . . . . . . . . . . . . . . . . . . . 1548

3.2 Richardson curves . . . . . . . . . . . . . . . . . . . . . . . . . . . . . 1548

3.3 Incidence coefficients . . . . . . . . . . . . . . . . . . . . . . . . . 1550

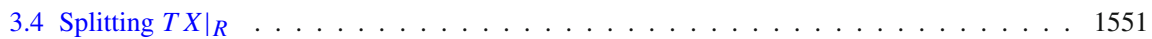

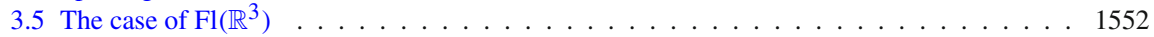

3.6 Decomposing $T W \ldots \ldots \ldots \ldots \ldots \ldots \ldots \ldots$

3.7 Determining the cycles . . . . . . . . . . . . . . . . . . . . . . 1557

3.8 Kocherlakota's theorem . . . . . . . . . . . . . . . . . . . . . . . 1558

3.9 Signs . . . . . . . . . . . . . . . . . . . . . . . . . . . 1559

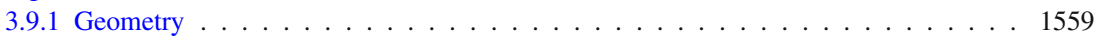

3.9 .2 Combinatorics . . . . . . . . . . . . . . . . . . . . . 1560

3.10 An example $\mathrm{Fl}\left(\mathbb{R}^{4}\right) \ldots \ldots \ldots \ldots \ldots \ldots \ldots \ldots$

4 The ring structure of $H^{*}\left(\mathrm{Fl}_{2 \mathcal{D}}^{\mathbb{R}} ; \mathbb{Q}\right)$ : Schubert calculus . . . . . . . . . . . . . . . . . 1564

5 Real Grassmannians . . . . . . . . . . . . . . . . . . . . . . . . . . . . . . . . . . 1567

5.1 Additive structure . . . . . . . . . . . . . . . . . . . . . . . . . . 1567

5.2 Multiplicative structure . . . . . . . . . . . . . . . . . . . . . . 1568

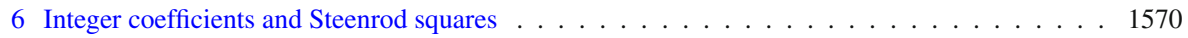

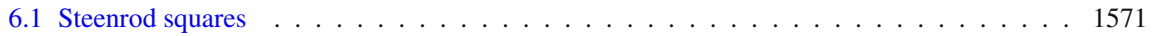

6.2 Integral cycles . . . . . . . . . . . . . . . . . . . . . . . . . . . . . . . . . . . . . . . . . . . . . . . . . . . . . . . . . . . .

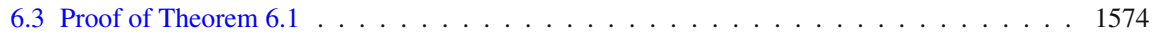

6.3 .1 Bockstein cohomology . . . . . . . . . . . . . . . . . . . . . . 1574

6.3 .2 Stabilization of Schubert classes . . . . . . . . . . . . . . . . . . 1575

6.3 .3 Bockstein cohomology of even flag manifolds . . . . . . . . . . . . . . . . . . . 1576

7 Enumerative applications: lower bounds . . . . . . . . . . . . . . . . . . . . . . . 1578

Appendix A. Topology: Cartan model . . . . . . . . . . . . . . . . . . . . . . . . . . . . . 1582

Appendix B. Schubert cycles: the general case of partial flag manifolds . . . . . . . . . . . . . . 1583

B. 1. The complete case . . . . . . . . . . . . . . . . . . . . . . . . . . 1584

B. 2. The odd case . . . . . . . . . . . . . . . . . . . . . . . . . . 1585

B. 3. The other cases . . . . . . . . . . . . . . . . . . . . . . . 1585

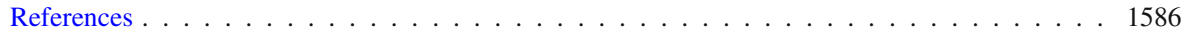

\section{Introduction}

In this paper we study the integer and rational coefficient cohomology of real partial flag manifolds.

\subsection{Additive structure}

\subsubsection{Incidence coefficients}

Complex flag manifolds have cell decompositions into even dimensional (complex) cells which makes the computation of CW-cohomology easy: all boundary maps are trivial. The generators of the cohomology groups can be represented by Schubert 
varieties whose multiplicative structure constants are given by Schubert calculus which is a classical and well-developed theory [36].

Real flag manifolds also have a cell decomposition, however the boundary maps are no longer trivial. The boundary maps have been first examined by Ehresmann [16]: he computed them completely for the case of real Grassmannians $\operatorname{Gr}_{p}\left(\mathbb{R}^{p+q}\right)=$ $\mathrm{Fl}_{p, q}^{\mathbb{R}}\left[16\right.$, p. 80], [13, p. 73], up to sign for flag manifolds of type $\mathrm{Fl}_{p, q, r}^{\mathbb{R}}[16, \mathrm{p}$. 85] and he determined the cycles in the case of $\mathrm{Fl}_{1, q, r}^{\mathbb{R}}[16, \mathrm{p} .87]$. In the case of Grassmannians, Pontryagin [44, Theorem 1] determined which Schubert varieties are cycles with rational coefficients (with some restrictions), see also [23, III.3.2.2.B] and [20]. Ehresmann also observed that the incidence coefficients are 0 or \pm 2 . This implies that the mod 2 cohomology groups of flag manifolds have an additive basis given by the Schubert cycles. Their mod 2 multiplicative structure constants follow from a theorem of Borel and Haefliger [7]: they agree with the structure constants of the complex Schubert cycles mod 2.

More generally, $R$-spaces (the flag manifolds of real semisimple Lie groups) have Bruhat cell decompositions [15]. If all multiplicities of the restricted roots are greater than 1, then there are no cells of neighboring dimensions [15]. In this case, the boundary relations of this cell decomposition are trivial, so additively the cohomology groups are freely generated by the closures of the Bruhat cells. If the multiplicities are not such, the boundary relations are no longer trivial, and to determine the cohomology groups, one has to compute the homology of a chain complex. Kocherlakota [38] computed the differentials in the Morse complex for general $R$-spaces up to sign. As he remarks, the open cells determined by the Morse function coincide with the Bruhat cells, so his computations determine the incidence coefficients which are 0 or \pm 2 .

In order to compute the integer or rational coefficient cohomology groups, the signs are also required. The latest development is the work of Rabelo and San Martin [46], who complete Kocherlakota's computation for $R$-spaces by determining the signs of the incidence coefficients via a CW homology approach.

Our first aim is to compute the incidence coefficients of the Schubert cells in real flag manifolds via a slightly different approach, namely by using the geometry of the Schubert cells. We give an alternative proof of Kocherlakota's theorem, then we compute the signs (Theorems 3.10, 3.13, 3.16). These results can be summarized as follows:

Theorem 3.10 The incidence coefficient of the Schubert cells $\Omega_{I}$ and $\Omega_{J}$ is given by

$$
\left[\Omega_{I}, \Omega_{J}\right]= \begin{cases}0, & N_{I}(a, b) \text { even } \\ (-1)^{s(I, J)} 2, & N_{I}(a, b) \text { odd }\end{cases}
$$

where $N_{I}(a, b)$ and $s(I, J)$ are integers determined by the combinatorics of the ordered set partitions I and J, see (2.1), (3.5) and Theorem 3.16.

We expect that the method presented here also generalizes to $R$-spaces, however in this paper we only consider the real partial flag manifolds $\mathrm{Fl}_{\mathcal{D}}^{\mathbb{R}}$. Our results are similar to [46], although the results are not directly comparable. Using Theorems 3.10 and 3.16, we computed several examples with SageMath's homology package [52]. Based on 
these computations, we formulated a conjecture stating that all torsion in $H^{*}\left(\mathrm{Fl}_{\mathcal{D}}^{\mathbb{R}} ; \mathbb{Z}\right)$ has order exactly 2 (see also Theorem 6.1).

\subsubsection{Cycles}

Once the incidence coefficients are known, it is a nontrivial combinatorial problem to determine what the integer or rational cohomology groups are, which Schubert varieties are cycles and what the further generators are (i.e. which union of oriented Schubert cells). This is currently unsolved for general real flag manifolds $\mathrm{Fl}_{\mathcal{D}}^{\mathbb{R}}$. The second contribution of this paper is that we determine which Schubert varieties are nonzero rational cycles in the case of even flag manifolds $\mathrm{Fl}_{2 \mathcal{D}}^{\mathbb{R}}$, see Theorem 3.12. In particular, we obtain the following result (for the notation, see (2.1) and Section 3.7).

Theorem 3.12 A basis of $H^{*}\left(\mathrm{Fl}_{2 \mathcal{D}}\left(\mathbb{R}^{2 N}\right) ; \mathbb{Q}\right)$ is given by

$$
H^{*}\left(\mathrm{Fl}_{2 \mathcal{D}}\left(\mathbb{R}^{2 N}\right) ; \mathbb{Q}\right)=\left\langle\left[\sigma_{D I}\right]: I \in\left(\begin{array}{l}
N \\
\mathcal{D}
\end{array}\right)\right\rangle .
$$

We also recover the result of Pontryagin on Schubert cycles in Grassmannians [44, Theorem 1], see Theorem 5.1. Using the incidence coefficients we also computed some small examples of geometric cycles generating $H^{*}\left(\mathrm{Fl}_{\mathcal{D}}^{\mathbb{R}} ; \mathbb{Q}\right)$ for general $\mathcal{D}$, see the tables of Appendix B. These tables illustrate the stark contrast of the general case with the simple descriptions of Theorems 3.12 and 5.1.

\subsection{Structure constants}

Once the cycles have been determined, the next step is to determine the multiplicative structure constants of the cycles. We will carry this out for $\mathrm{Fl}_{2 \mathcal{D}}^{\mathbb{R}}$ by showing that they are circle spaces with rational coefficient cohomology [17,41], see Theorem 4.4, its Corollaries 4.5, 4.6. Namely, in Corollary 4.5 we obtain the following result.

Theorem (Corollary 4.5) The structure constants of $\left[\sigma_{D I}^{\mathbb{R}}\right] \in H^{*}\left(\mathrm{Fl}_{2 D}^{\mathbb{R}} ; \mathbb{Q}\right)$ agree with the structure constants of $\left[\sigma_{I}^{\mathbb{C}}\right] \in H^{*}\left(\mathrm{Fl}_{\mathcal{D}}^{\mathbb{C}} ; \mathbb{Q}\right)$ :

$$
\left[\sigma_{D I}^{\mathbb{R}}\right] \cdot\left[\sigma_{D J}^{\mathbb{R}}\right]=\sum_{K} c_{I J}^{K}\left[\sigma_{D K}^{\mathbb{R}}\right] \quad \Longleftrightarrow \quad\left[\sigma_{I}^{\mathbb{C}}\right] \cdot\left[\sigma_{J}^{\mathbb{C}}\right]=\sum_{K} c_{I J}^{K}\left[\sigma_{K}^{\mathbb{C}}\right]
$$

In the special case of Grassmannians, this follows from results of [23, III.3.2.3.E], and [20], see also Propositions 5.2, 5.3. In the context of algebraic geometry, the ChowWitt rings of real Grassmannians have been recently considered in [57]; the similarity of Propositions 5.2, 5.3 with [57, Theorem 1.2] suggests that Corollaries 4.5, 4.6 have analogues for the Chow-Witt rings of even flag manifolds.

\subsection{Characteristic classes}

Returning to the complex case, another kind of description of the cohomology ring of the complex flag manifolds is given in terms of characteristic classes of their tautologi- 
cal bundles. Namely, $H^{*}\left(\mathrm{Fl}_{\mathcal{D}}\left(\mathbb{C}^{N}\right) ; \mathbb{Z}\right)$ is generated as an algebra by the Chern classes $c_{i}\left(D_{j}\right)$ of the tautological quotient bundles $D_{j}=S_{j} / S_{j-1}$. In modern language, this can be formulated as surjectivity of the Kirwan map [35]. The relations are given by the identity $\prod_{j=1}^{m} c_{*}\left(D_{j}\right)=1$, where $c_{*}$ is the total Chern class, see e.g. [8, Chapter 23]. In the case of the Grassmannians, the relationship between these two descriptions is given by the Giambelli formula

$$
\left[\sigma_{\lambda}\right]=\operatorname{det}\left(c_{\lambda_{i}+j-i}(Q)\right)
$$

In the real case $G=\mathrm{GL}(N, \mathbb{R})$, and a parabolic subgroup $P \leq G$, Pontryagin classes do not always generate the cohomology $\operatorname{ring} H^{*}(G / P ; \mathbb{Q})$; this is only the case if $G$ and $P$ have the same rank, i.e. even real flag manifolds $\mathrm{Fl}_{2 D}^{\mathbb{R}}$. In other words, the "rational real Kirwan map" is surjective iff $\mathrm{rk} P=\mathrm{rk} G$. In this case, we express $\left[\sigma_{\lambda}\right]$ in terms of Pontryagin classes, see Corollary 4.6.

Casian and Kodama [11] made a conjecture about the ring structure of $H^{*}\left(\mathrm{Fl}_{\mathcal{D}}^{\mathbb{R}} ; \mathbb{Q}\right)$ in the case of $\mathcal{D}=(k, n-k)$, i.e. Grassmannians $H^{*}\left(\operatorname{Gr}_{k}\left(\mathbb{R}^{n}\right) ; \mathbb{Q}\right)$, which has been proved even equivariantly via different approaches, see [10,30,47,50]. Recently, He [32] determined the cohomology ring $H^{*}\left(\mathrm{Fl}_{\mathcal{D}}^{\mathbb{R}} ; \mathbb{Q}\right)$ for arbitrary $\mathcal{D}$. We state He's theorem in the form convenient for us in Theorem A.3.

\subsection{Summary of the results on $H^{*}\left(\mathbf{F I} I_{\mathcal{D}}^{\mathbb{R}} ; \mathbb{Q}\right)$}

The new results of this paper partially answer the following questions. Given a real partial flag manifold $\mathrm{Fl}_{\mathcal{D}}^{\mathbb{R}}$ :

(Q1) Which Schubert varieties $\sigma_{I}$ are cycles? $\left(I \in\left(\begin{array}{l}N \\ \mathcal{D}\end{array}\right)\right.$, see $\left.(2.1)\right)$ Which ones are nonzero in $H^{*}\left(\mathrm{Fl}_{\mathcal{D}}^{\mathbb{R}} ; \mathbb{Q}\right)$ ? Which linear combinations of Schubert cells are the remaining generators?

(Q2) What are the multiplicative structure constants of the cycles?

(Q3) What are the relations between Pontryagin classes of the tautological bundles? What further additional generators $r_{i}$ are there and what are the relations?

(Q4) How to express one set of generators from the other? $\sigma_{I}\left(p_{i}, r_{i}\right)=$ ? $p_{i}\left(\sigma_{I}\right)=$ ?, $r_{i}\left(\sigma_{I}\right)=$ ?

Theorems 3.12, 5.1 and Appendix B are results of type (Q1). Theorem 4.4, Corollary 4.5, and Propositions 5.2, 5.3 concern (Q2). He's Theorem A.3 [32] answers (Q3) in rational coefficient cohomology. Corollary 4.6 concerns (Q4).

Note, that (Q1), (Q3) and (Q4) imply (Q2) rationally, at least in theory; in practice giving combinatorial rules to compute the structure constants is not immediate and has been extensively studied in the complex case for different kind of cohomology theories by Littlewood-Richardson rules, checkers, puzzles [25,37,53]. By Corollary 4.5 , the same combinatorial descriptions can be applied in the even real case $\mathrm{Fl}_{2 D}^{\mathbb{R}}$. 


\subsection{Integer coefficients}

The final step is determining the integer coefficient cohomology. The formulas for the incidence coefficients (Theorems 3.10,3.16) can be used in a computational homology program, to compute the cohomology groups and their generators. We calculated several examples using SageMath's homology package (all partial flag manifolds of $\mathbb{R}^{N}, N \leq 7$ and some up to $\mathbb{R}^{11}$, see Appendix B for some of the results). Based on these computations, we make the following conjecture:

Conjecture Every torsion element in $H^{*}\left(\mathrm{Fl}_{\mathcal{D}}^{\mathbb{R}} ; \mathbb{Z}\right)$ is of order exactly two.

If the conjecture is true, then the cohomology groups can be completely determined (cf. Proposition 6.2). The conjecture is known in the following cases. For infinite Grassmannians it is classical [6] that all torsion is of order 2. For finite Grassmannians, this is a result of Ehresmann [16]. We prove the following result:

Theorem 6.1 Every torsion element in $H^{*}\left(\mathrm{Fl}_{2 D}^{\mathbb{R}} ; \mathbb{Z}\right)$ is of order exactly two.

In particular, the integral classes of the Schubert cycles $[Z]$ are completely determined by their rational and mod 2 reductions. The proof of this theorem involves computing the Bockstein cohomology of $X:=\mathrm{Fl}_{2 \mathcal{D}}^{\mathbb{R}}$ :

$$
H_{\beta}^{*}(X):=\left(H^{*}\left(X ; \mathbb{F}_{2}\right) ; \mathrm{Sq}^{1}\right),
$$

$\left(\mathrm{Sq}^{1} \circ \mathrm{Sq}^{1}=0\right) ; H_{\beta}^{*}(X)$ is the first page of the Bockstein Spectral Sequence. In the computations, we use that $\mathrm{Sq}^{1}\left[\sigma_{I}\right]$ equals the sum of those Schubert classes $\left[\sigma_{J}\right]$ which have nonzero incidence coefficient with $\left[\sigma_{I}\right]$ (Proposition 6.3) this extends an observation of Lenart [40]. This concludes the determination of the cohomology groups $H^{*}\left(\mathrm{Fl}_{2 \mathcal{D}} ; \mathbb{Z}\right)$. We do not take on the task of determining the integer coefficient ring structure.

\subsection{Applications - real enumerative geometry}

Using the Schubert cycle description of the rational cohomology ring structure, we give an application to real enumerative geometry. Whereas in the complex case, the answer to an enumerative geometry problem is a single number, in the real case, the answer is a list of possible numbers, depending on the generic configuration. In general, very little is known about the complete range of such numbers. In the case of flag manifolds, the enumerative geometric problems are called Schubert problems and in general, the range of possible solutions is unknown. However, the cohomology ring calculation provides a lower bound. In many cases, the lower bound is 0 , however in some cases, there is a meaningful lower bound, see Proposition 7.1.

\section{Preliminaries}

In this section we recall the general definition of the Vassiliev complex and introduce some notation for the geometry of real flag manifolds. 


\subsection{Vassiliev complex - incidence coefficients}

Throughout this section let $\left(X_{\alpha}\right)_{\alpha \in A}$ be a stratification of a smooth manifold $X$ where each stratum is contractible. Vassiliev [55], [2, Ch. 4.2] gave a method for computing the cohomology of $X$ and determining when the closure of a stratum has a fundamental cohomology class (or in another terminology is a 'cycle'). Let $F_{i}:=X \backslash \overline{X^{i+1}}$ be the open codimension filtration of $X$, where $X^{i}$ is the union of the $i$-codimensional strata. The Vassiliev complex is the bottom row of the $E_{1}$-page of the spectral sequence associated to this filtration with connecting homomorphism

$$
d: H^{p}\left(F_{p}, F_{p} \backslash X^{p}\right) \rightarrow H^{p+1}\left(F_{p+1}, F_{p+1} \backslash X^{p+1}\right),
$$

The cohomology of the Vassiliev complex computes $H^{*}(X ; \mathbb{Z})$, since the spectral sequence of the filtration degenerates on the $E_{2}$-page by the contractibility assumption on the strata. Furthermore, since the strata are contractible, their normal bundles are trivial; let us fix a coorientation on each stratum. The $k$-cochains in the Vassiliev complex are the linear combinations of the $k$-codimensional strata $X_{\alpha}$ with their fixed coorientation, and the differential $d$ can be written as

$$
d X_{\alpha}=\sum_{\operatorname{codim} X_{\beta}=\operatorname{codim} X_{\alpha}+1} n_{\alpha \beta} X_{\beta} .
$$

We will also use the notation $\left[X_{\alpha}, X_{\beta}\right]$ instead of $n_{\alpha \beta}$. In case one can find a submanifold $D$ of $X$ intersecting all strata of $\overline{X_{\alpha}}$ transversally, one can compute the incidence coefficients $n_{\alpha \beta}$ geometrically as follows ( [2,55], see also [19]). If $X_{\beta} \nsubseteq \overline{X_{\alpha}}$ then $n_{\alpha \beta}=0$.

Let $A:=X_{\alpha}$ be a $k$-codimensional and $B:=X_{\beta} \subseteq \overline{X_{\alpha}}$ be a $(k+1)$-codimensional stratum, with normal bundles $v_{\alpha}, v_{\beta}$ respectively (these are trivial by the contractibility assumption). Let $D:=D^{k+1}$ be a $(k+1)$-dimensional submanifold of $X$ that intersects all strata of $\bar{A}$ transversally and intersects $B$ in a point $b$. Let $L:=D \cap A$ which by transversality is a disjoint union of connected curves $L_{i}$, whose closure contains $b$. For each $L_{i}$, choose a splitting $s$ of the quotient map $q:\left.\left.T X\right|_{L_{i}} \rightarrow v_{\alpha}\right|_{L_{i}}$, such that

$$
\left.T D\right|_{L_{i}}=\left.T L_{i} \oplus v_{\alpha}\right|_{L_{i}},
$$

where $\left.\left.v_{\alpha}\right|_{L_{i}} \subseteq T X\right|_{L_{i}}$ via the splitting $s$. Then $T L_{i}$ is oriented by taking the orientation pointing towards $b$ and $v_{\alpha}$ is oriented by the coorientation of $A$, so they determine an orientation of $\left.T D\right|_{L_{i}}$ (fix the convention of taking $T L_{i}$ first, then $\left.v_{\alpha}\right|_{L_{i}}$ ). This extends to an orientation $O_{1}$ of $T D$ at $b$. Since $D$ is transversal to $B$, the orientation of $\left.v_{\beta}\right|_{b}$ determines an orientation $O_{2}$ of $\left.T D\right|_{b}$. If the two orientations $O_{1}$ and $O_{2}$ agree for $L_{i}$, then set $n_{\alpha \beta}^{i}:=+1$, otherwise -1 . Then $n_{\alpha \beta}=\sum_{i} n_{\alpha \beta}^{i}$.

One can show that $X_{\alpha}$ is a cycle in the Vassiliev complex, iff $Z=\overline{X_{\alpha}}$ has a fundamental cohomology class: from now on we will simply say that $Z$ is a cycle. We will compute the Vassiliev complex of real partial flag manifolds for the stratification by Schubert cells in Sect. 3 . 


\subsection{Geometry of flag manifolds}

This section is standard, see $[9,25]$ for the complex case. We include it to fix some notation and properties that we will use in the computations of Sect. 3. We are interested in the real case, so $\operatorname{GL}(N)$ denotes $\operatorname{GL}(N, \mathbb{R})$ and $B^{+}$is the subgroup of real upper triangular matrices.

\subsubsection{Schubert varieties, orbit structure}

Denote the standard basis in $\mathbb{R}^{N}$ by $e_{1}, \ldots, e_{N}$, their one-dimensional spans by $\varepsilon_{i}=$ $\left\langle e_{i}\right\rangle$, and let $E_{\bullet}$ be the standard flag, which consists of the subspaces $E_{j}=\bigoplus_{i=1}^{j} \varepsilon_{i}$. The stabilizer of $E_{\bullet}$ in $G:=\mathrm{GL}(N)$ is $B^{+}$. Choose a parabolic subgroup, i.e. $B^{+} \subseteq$ $P \subseteq G$. Similarly to the complex case,

$$
P=\operatorname{GL}(\mathcal{D}), \quad \text { for some } \mathcal{D}=\left(d_{1}, \ldots, d_{m}\right), \sum d_{i}=N
$$

which is the subgroup of block upper-triangular matrices with elements of GL $\left(d_{i}\right)$ on the diagonal blocks and arbitrary entries above the blocks.

The corresponding homogeneous space $X=G / P$ is the partial flag manifold $\mathrm{Fl}_{\mathcal{D}}\left(\mathbb{R}^{N}\right)$. Using this notation, $d_{i}$ denotes the difference in the dimensions of the flags. Let their dimensions be $\mathcal{S}=\left(s_{1}, \ldots, s_{m}\right)$, where $s_{1}=d_{1}$, and $s_{i}-s_{i-1}=d_{i}$ for $i>1$.

The $B^{+}$-orbits on $X$ are called Bruhat cells $\Omega_{I}\left(E_{\bullet}\right)$. Each of these contains a unique coordinate flag $E_{\bullet}^{I} \in \mathrm{Fl}_{\mathcal{D}}\left(\mathbb{R}^{N}\right)$ (2.2) by the Bruhat decomposition [9, Section 1.2] or [15, Corollary 3.8] for the real case. These in turn are indexed by ordered set partitions $I \in \operatorname{OSP}(\mathcal{D})$, where

$$
\operatorname{OSP}(\mathcal{D}):=\left(\begin{array}{l}
N \\
\mathcal{D}
\end{array}\right)=S_{N} /\left(S_{d_{1}} \times \ldots \times S_{d_{m}}\right)
$$

By definition, an element of $\operatorname{OSP}(\mathcal{D})$ is a partition of the numbers $[N]:=\{1, \ldots, N\}$ to $m$ parts of sizes $d_{1}, \ldots, d_{m}$. In particular, the $j$ th part is a subset of $[N]$ of size $d_{j}$, that we will denote by $I_{j} \in\left(\begin{array}{l}N \\ d_{j}\end{array}\right)$. In general, we will denote the set of $k$-element subsets of $N$ by $\left(\begin{array}{l}N \\ k\end{array}\right)$. Using this indexing, for $I \in \operatorname{OSP}(\mathcal{D})$, we denote by $E_{\bullet}^{I}$ the coordinate flag $\left(E_{1}^{I} \subseteq E_{2}^{I} \subseteq \ldots \subseteq E_{m}^{I}\right.$ ) whose $r$ th term is

$$
E_{r}^{I}=\bigoplus_{\substack{j \leq r \\ l \in I_{j}}} \varepsilon_{l} .
$$

Notation. We will represent $I \in \operatorname{OSP}(\mathcal{D})$ by the minimal length element in $S_{N}$ in the coset of $I$ : list elements of $I_{1}$ in increasing order, then elements of $I_{2}$ in increasing order etc. - the $I_{j}$ separated by brackets or commas. In particular, for complete flag manifolds $\mathcal{D}=\left(1^{N}\right)$ this coincides with the one-line notation of $\operatorname{OSP}(\mathcal{D})=S_{N}$, 
the same convention as [25], see also [26, p. 20]. It is sometimes convenient to write $I \in\left(\begin{array}{l}N \\ \mathcal{D}\end{array}\right)$ as a function: $I:[N] \rightarrow[m]$ satisfying $\left|I^{-1}(j)\right|=d_{j}$ for all $j$.

Given a general complete flag $A_{\bullet}$, the Bruhat cells coincide with the following Schubert cell description:

$$
\Omega_{I}\left(A_{\bullet}\right)=\left\{F_{\bullet} \in \mathrm{Fl}_{\mathcal{D}}\left(\mathbb{R}^{N}\right): \operatorname{dim} F_{i} \cap A_{k}=r_{I}(i, k)\right\},
$$

where $r_{I}(i, k)=\left|\left\{l \in I_{1} \cup \ldots \cup I_{i}: l \leq k\right\}\right|$. When we omit the flag from the notation $\Omega_{I}$, that means that we take the standard flag $E_{\bullet}$. For the dimension of $\Omega_{I}$, $I \in \operatorname{OSP}(\mathcal{D})$, introduce $\ell(I)$ to be the number of inversions (i.e. pairs of elements in reverse order):

$$
\ell(I):=\left|\left\{(a, b): a>b, a \in I_{\alpha}, b \in I_{\beta}, \alpha<\beta\right\}\right| .
$$

Then $\operatorname{dim} \Omega_{I}=\ell(I)$ (see Proposition 2.3).

The closure of the orbit $\Omega_{I}$ is called a Schubert variety and is denoted $\sigma_{I}$. If a Schubert variety $\sigma_{I}$ is a cycle (in the sense discussed in Sect. 2.1), we call it a Schubert cycle and its class $\left[\sigma_{I}\right]$ a Schubert class. The orbit structure is described by the Bruhat order (cf. [38, Theorem 2.3.2] for the real case):

$$
\sigma_{I}=\bigcup_{J \leq I} \Omega_{J}
$$

where $J \leq I$ iff $\left(J_{1} \cup \ldots \cup J_{i}\right)_{\text {rtiv }} \leq\left(I_{1} \cup \ldots \cup I_{i}\right)_{\text {rtiv }}$ for all $i$, where rtiv means "reordered to increasing value" and the partial order $\left(a_{1}, \ldots, a_{j}\right) \leq\left(b_{1}, \ldots, b_{j}\right)$ is the lexicographic one. This is also equivalent to $r_{I}(i, k) \geq r_{J}(i, k)$ for all $i, k$.

\subsubsection{Tangent bundle of $\mathrm{FI}_{\mathcal{D}}\left(\mathbb{R}^{N}\right)$}

We recall a well-known decomposition of the tangent bundle of $X$ in terms of tautological bundles.

Let $G:=\mathrm{GL}(N)$ and $P \subseteq G$ be a parabolic subgroup; $P=\mathrm{GL}(\mathcal{D})$. $P$ has projections to subgroups $p_{i}: P \rightarrow \mathrm{GL}\left(s_{i}\right)$ which are homomorphisms, whose defining representations induce the tautological bundles. For example, the defining representation of $\operatorname{GL}\left(s_{i}\right)$ on $\mathbb{R}^{s_{i}}$ induces the $i$ th tautological bundle over $G / P$ :

$$
S_{i} \cong \mathrm{GL}(N) \times{ }_{P} \mathbb{R}^{s_{i}}
$$

The quotient and difference bundles are defined by the following exact sequences of bundles over $X$ :

$$
\begin{aligned}
& 0 \longrightarrow S_{i} \longrightarrow \mathbb{R}^{N} \longrightarrow Q_{i} \longrightarrow 0 \\
& 0 \longrightarrow S_{i-1} \longrightarrow S_{i} \longrightarrow D_{i} \longrightarrow 0
\end{aligned}
$$


with the convention $S_{0}=0$. Notice that $s_{i}=\operatorname{dim} S_{i}, d_{i}=\operatorname{dim} D_{i}$, and let $q_{i}=$ $\operatorname{dim} Q_{i}$. Recall the following general fact about the tangent bundle of homogeneous spaces:

Proposition 2.1 [27, Lemma II.2.3.1. (p. 132)] Let $X=G / H$ be a homogeneous space and let $\mathfrak{g}$ and $\mathfrak{h}$ denote the Lie algebras of $G$ and $H$ respectively. Then the $G$ equivariant vector bundle $T X \rightarrow X$ fits into the short exact sequence of $G$-equivariant bundles

$$
0 \rightarrow G \times_{H} \mathfrak{h} \rightarrow G \times_{H} \mathfrak{g} \rightarrow G \times_{H}(\mathfrak{g} / \mathfrak{h}) \cong T X \rightarrow 0
$$

where $H$ acts on $\mathfrak{g}, \mathfrak{h}$ via the adjoint representation.

\section{Corollary 2.2}

$$
T X \cong \bigoplus_{i=1}^{m-1} \operatorname{Hom}\left(D_{i}, Q_{i}\right) \cong \bigoplus_{1 \leq i<j \leq m} \operatorname{Hom}\left(D_{i}, D_{j}\right)
$$

Proof Apply the Proposition to the homogeneous space $\mathrm{Fl}_{\mathcal{D}}\left(\mathbb{R}^{N}\right)=\mathrm{GL}(N) / \mathrm{GL}(\mathcal{D})$.

A choice of the basis $e_{i} \in \mathbb{R}^{N}$ induces splittings $Q_{i} \rightarrow \mathbb{R}^{N}, D_{i} \rightarrow \mathbb{R}^{N}$, which is not essential, but facilitates computations, in particular it realizes $T X$ as a subbundle of $\operatorname{End}\left(\mathbb{R}^{N}\right)$. To alleviate notation, from now on, we will use an abuse of notation and write $N_{I} \Omega_{I}$ instead of $N_{E_{\bullet}^{I}} \Omega_{I}$, where $E_{\bullet}^{I}$ is the coordinate flag corresponding to the ordered set partition $I$ introduced in (2.2). Then the tangent and normal spaces of $\Omega_{I}$ can be identified as follows:

Proposition 2.3 The tangent and normal spaces of $\Omega_{I}$ at $E_{\bullet}^{I}, I \in \operatorname{OSP}(\mathcal{D})$ are given by

$$
T_{I} \Omega_{I}=\bigoplus_{(c, d) \in T_{I}} \varepsilon_{c d}, \quad N_{I} \Omega_{I}=\bigoplus_{(c, d) \in N_{I}} \varepsilon_{c d}
$$

where $\varepsilon_{c d}=\operatorname{Hom}\left(\varepsilon_{c}, \varepsilon_{d}\right)$ and

$T_{I}:=\left\{(c, d) \in[N]^{2}: c>d, I(c)<I(d)\right\}, \quad N_{I}:=\left\{(c, d) \in[N]^{2}: c<d, I(c)<I(d)\right\}$.

In particular, the dimension of $\Omega_{I}$ is given by $\left|T_{I}\right|=\ell(I)$.

Using the identification $T X \subseteq \operatorname{End}\left(\mathbb{R}^{N}\right)$ as a subbundle and Corollary 2.2, the proof consists of computing the stabilizer subgroups $\operatorname{Stab}_{B^{+}}\left(E_{\bullet}^{I}\right)$, we omit the details.

\subsubsection{Direct sum maps}

We are going to make use of the following natural maps between flag manifolds. Let $\mathcal{D}_{1}=\left(d_{i}^{1}\right) \in \mathbb{N}^{m}, \mathcal{D}_{2}=\left(d_{i}^{2}\right) \in \mathbb{N}^{m}$ be two ordered sets of natural numbers, where 
we allow zero and let $\mathcal{D}_{1}+\mathcal{D}_{2}=\left(d_{i}^{1}+d_{i}^{2}\right)$ be their element-wise sum. Let $A_{1}$ be a vector space of dimension $\sum d_{i}^{1}$, and let $A_{2}$ be a vector space of dimension $\sum d_{i}^{2}$. The direct sum of $A_{1}$ and $A_{2}$ induces the following direct sum map of flag manifolds:

$$
F_{\mathcal{D}_{1}, \mathcal{D}_{2}}: \mathrm{Fl}_{\mathcal{D}_{1}}\left(A_{1}\right) \times \mathrm{Fl}_{\mathcal{D}_{2}}\left(A_{2}\right) \hookrightarrow \mathrm{Fl}_{\mathcal{D}_{1}+\mathcal{D}_{2}}\left(A_{1} \oplus A_{2}\right)
$$

defined by

$$
F_{\mathcal{D}_{1}, \mathcal{D}_{2}}\left(F_{\bullet}^{1}, F_{\bullet}^{2}\right)_{\kappa}:=F_{\kappa}^{1} \oplus F_{\kappa}^{2}
$$

A direct sum decomposition $V=\bigoplus_{i} A_{i}$ induces a direct sum decomposition

$$
\operatorname{End}(V)=\bigoplus_{i, j} \operatorname{Hom}\left(A_{i}, A_{j}\right),
$$

in particular we obtain inclusions $\iota_{j}: \operatorname{End}\left(A_{j}\right) \hookrightarrow \operatorname{End}(V)$.

Proposition 2.4 Let $\mathrm{Fl}_{\mathcal{D}_{i}}\left(A_{i}\right), i=1,2$ be two flag manifolds with $\mathcal{D}_{i} \in \mathbb{N}^{m}$, and let $E_{\bullet}^{2} \in \mathrm{Fl}_{\mathcal{D}_{2}}\left(A_{2}\right)$ be a fixed flag. Then

$$
f: \mathrm{Fl}_{\mathcal{D}_{1}}\left(A_{1}\right) \rightarrow \mathrm{Fl}_{\mathcal{D}_{1}+\mathcal{D}_{2}}\left(A_{1} \oplus A_{2}\right)
$$

defined by $f:=F_{\mathcal{D}_{1}, \mathcal{D}_{2}}\left(\cdot, E_{\bullet}^{2}\right)$ is an isomorphism onto its image, and

$$
d f: T \mathrm{Fl}_{\mathcal{D}_{1}} \rightarrow T \mathrm{Fl}_{\mathcal{D}_{1}+\mathcal{D}_{2}}
$$

coincides with $\left.\iota_{1}\right|_{T \mathrm{Fl}_{\mathcal{D}_{1}}}$, where $T \mathrm{Fl}_{\mathcal{D}_{1}} \subseteq \operatorname{End}\left(A_{1}\right)$ and $T \mathrm{Fl}_{\mathcal{D}_{1}+\mathcal{D}_{2}} \subseteq \operatorname{End}\left(A_{1} \oplus A_{2}\right)$ are subbundles using the identification determined by the bases $\left(a_{i}^{1} \in A_{1}\right),\left(a_{i}^{2} \in A_{2}\right)$.

Proof (Sketch of proof) The direct sum map is a $\operatorname{GL}\left(A_{1}\right) \times \operatorname{GL}\left(A_{2}\right) \subseteq \operatorname{GL}\left(A_{1} \oplus A_{2}\right)$ equivariant embedding, which implies the first statement. Denote the tautological difference bundles over $\mathrm{Fl}_{\mathcal{D}_{i}}\left(A_{i}\right)$ by $D_{i}^{j}, i=1, \ldots, m, j=1,2$ and over $\mathrm{Fl}_{\mathcal{D}_{1}+\mathcal{D}_{2}}\left(A_{1} \oplus A_{2}\right)$ denote them by $D_{i}, i=1, \ldots, m$. Using the splittings $D_{i}^{j} \hookrightarrow A_{j}$ and $D_{i} \hookrightarrow A_{1} \oplus A_{2}$ for $i=1, \ldots, m, j=1,2$ :

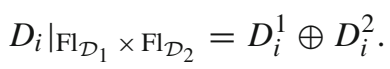

The second part of the claim then follows from commutativity of the following diagram of bundles over $\mathrm{Fl}_{\mathcal{D}_{1}}$ :

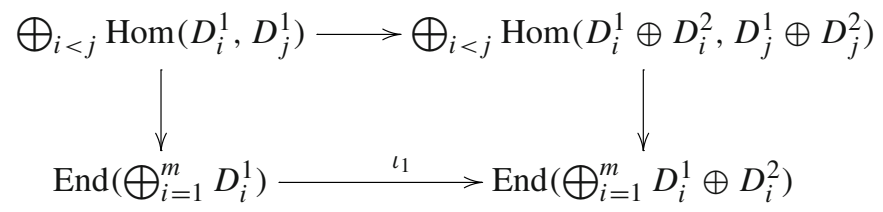




\section{The Vassiliev complex of $\mathrm{FI}_{\mathcal{D}}^{\mathbb{R}}$}

In this section we compute the Vassiliev complex of $\mathrm{Fl}_{\mathcal{D}}^{\mathbb{R}}$ as described in Sect. 2.1, whose cohomology computes $H^{*}\left(\mathrm{Fl}_{\mathcal{D}}^{\mathbb{R}} ; \mathbb{Z}\right)$. The stratification is given by the Schubert cells $\Omega_{I}$. Explicitly, we determine the incidence coefficients $\left[\Omega_{I}, \Omega_{J}\right]$. To compute the coefficients $\left[\Omega_{I}, \Omega_{J}\right]$, one has to coorient each $\Omega_{I}$, and compare these coorientations by extending them to the adjacent orbits $\Omega_{J}$ along transversal submanifolds $D$ as described in Sect. 2.1. The computations have many similarities to the one given by Kocherlakota, but instead of Morse theory we emphasize the geometry of the Schubert cells. Let us give a brief outline of the proof.

In Sect. 3.1 we define the coorientation of the Schubert cells $\Omega_{I}$. In Sect. 3.2 we define the transversal submanifolds $D$ to be the opposite Bruhat cells. The intersection of $W:=\Omega_{I} \cup \Omega_{J}$ with $D$ is a Richardson curve $R \cong \mathbb{R} P^{1}$. In Sect. 3.3 we show that $\left[\Omega_{I}, \Omega_{J}\right]$ is 0 or \pm 2 depending on whether the restriction of the normal bundle $\left.v(W)\right|_{R}$ is trivial or not. To determine triviality of $\left.v(W)\right|_{R}$ we decompose it into a direct sum of line bundles, which is obtained in Sects. 3.4-3.6. This yields a combinatorial description of $\left[\Omega_{I}, \Omega_{J}\right]$ in terms of the number of Möbius bundles over $R$. In Sect. 3.7 using this combinatorial description, we determine which Schubert varieties $\sigma_{I}$ are nonzero rational cycles in the even case $\mathrm{Fl}_{2 \mathcal{D}}^{\mathbb{R}}$. In Sect. 3.8 we relate our computations to the theorem of Kocherlakota. By considering local orientations of $\left.v(W)\right|_{R}$, we determine the sign of $\left[\Omega_{I}, \Omega_{J}\right]$ in Sect. 3.9. To conclude the chapter we illustrate the results on $\mathrm{Fl}\left(\mathbb{R}^{4}\right)$. We will use the notation of Sect. 2.2.

We remark that in the case of real (and complex) flag manifolds, each $B^{+}$-orbit is homeomorphic to an affine space, so the orbit stratification yields a cell decomposition. Therefore computing the incidence coefficients agrees with the incidence coefficients of the CW complex, which have been examined by Ehresmann [16] for Grassmannians, later by Kocherlakota for generalized real flag manifolds ( $R$-spaces) using the Morse complex [38] and most recently by Rabelo and San Martin [46] using CW homology (for the general case of $R$-spaces).

\subsection{Coorientation of the strata}

We describe the Vassiliev complex of $X=\mathrm{Fl}_{\mathcal{D}}\left(\mathbb{R}^{N}\right), \mathcal{D}=\left(d_{1}, \ldots, d_{m}\right)$. First, we coorient all cells by fixing a coorientation of $\Omega_{I}$ at $E_{\bullet}^{I}$ (see Sect. 2.2 for the notation). Using the decomposition of the tangent and normal spaces given in Proposition 2.3, orient both the tangent and normal spaces by the lexicographic ordering of those $e_{k l}=\left(e_{k} \mapsto e_{l}\right)$ which appear in them. Since $\Omega_{I}$ is contractible, the orientation of the normal space $N_{I} \Omega_{I}$ at $E_{\bullet}^{I}$ determines a coorientation on the whole of $\Omega_{I}$. In fact, we will not make use of the choice of orientations up until Sect. 3.9 when we determine signs of the incidence coefficients.

\subsection{Richardson curves}

Our aim is to determine the incidence numbers $\left[\Omega_{I}, \Omega_{J}\right]$, for $\ell(J)=\ell(I)-1$ and $J \leq I$ (recall the notations (2.4) and (2.5)). The Bruhat order implies that $J$ is obtained 
from $I$ by interchanging $a \in I_{\alpha}$ with some $b \in I_{\beta}, a>b, \alpha<\beta$ (this follows e.g. from [38, Theorem 2.3.2]). We call such $I, J$ (and $\Omega_{I}, \Omega_{J}$ ) adjacent and fix this data in the upcoming discussion.

According to the construction of the Vassiliev complex (Sect. 2.1), we will fix a transversal submanifold to $\Omega_{J}$ at $E_{\bullet}^{J}$; natural candidates are the dual Schubert cells, i.e. the orbits of the opposite Borel subgroup $B^{-}$. The $B^{-}$-orbits $B^{-} E_{\bullet}^{J}$ have the following characterization:

$$
B^{-} E_{\bullet}^{J}=\Omega_{J^{D}}\left(E_{\bullet}^{\vee}\right)=\left\{F_{\bullet} \in \mathrm{Fl}_{\mathcal{D}}\left(\mathbb{R}^{N}\right): \operatorname{dim} F_{i} \cap E_{k}^{\vee}=r_{J^{D}}(i, k)\right\}
$$

where $J^{D}=\left(J_{1}^{D}, J_{2}^{D}, \ldots, J_{m}^{D}\right), J_{i}^{D}:=N+1-J_{i}$ and $E_{\bullet}^{\vee}$ is the standard dual flag, whose $i$ th subspace is

$$
E_{i}^{\vee}=\left\langle e_{N}, \ldots, e_{N-i+1}\right\rangle
$$

Since the flags $E_{\bullet}, E_{\bullet}^{\vee}$ are transverse, all $B^{-}$-orbits are transverse to $\Omega_{I}$, so $B^{-} E_{\bullet}^{J}$ is a transversal submanifold to $\Omega_{J}$ at $E_{\bullet}^{J}$. For general $I, J$, the intersections $\sigma_{I}^{J}=\sigma_{I}\left(E_{\bullet}\right) \cap \sigma_{J^{D}}\left(E_{\bullet}^{\vee}\right)$ are called Richardson varieties. To determine the incidence numbers $\left[\Omega_{I}, \Omega_{J}\right]$, we will be interested in the Richardson curves $\sigma_{I}^{J}$ when $\ell(J)=\ell(I)-1$ and $J \leq I$. Intuitively, the Richardson curve is the curve between the coordinate flags $E_{\bullet}^{I}$ and $E_{\bullet}^{J}$ obtained by continuously exchanging the coordinates $\varepsilon_{a}$ and $\varepsilon_{b}$ in $E_{\bullet}^{I}$.

More precisely, in terms of the direct sum maps of Proposition 2.4, the Richardson curve $\sigma_{I}^{J}$ is the isomorphic image of $f: \mathbb{P}\left(A_{1}\right) \hookrightarrow \mathrm{Fl}_{\mathcal{D}}\left(\mathbb{R}^{N}\right)$ for $A_{1}:=\varepsilon_{a} \oplus \varepsilon_{b}$, $A_{2}:=A_{1}^{\vee}=\bigoplus_{i \neq a, b} \varepsilon_{i}$ and $E_{\bullet}:=E_{\bullet}^{I} \cap E_{\bullet}^{J}$,

$$
f(\cdot)=F_{\mathcal{D}_{1}, \mathcal{D}_{2}}\left(\cdot, E_{\bullet}\right), \quad \mathcal{D}_{2}=\left(d_{1}, \ldots, d_{\alpha}-1, \ldots, d_{\beta}-1, \ldots, d_{m}\right),
$$

for $\mathcal{D}=\mathcal{D}_{1}+\mathcal{D}_{2}$ and $\mathcal{D}_{1}=(1,1)$ in positions $\alpha, \beta$. Note that $\sigma_{I}^{J}$ is isomorphic to $\mathbb{R} P^{1}$. The isomorphism $f$ induces tautological bundles $\rho \rightarrow \sigma_{I}^{J}$ on the Richardson curves as follows. Let

$$
\tilde{f}=(\mathrm{id}, f): A_{1} \times \mathbb{P}\left(A_{1}\right) \rightarrow A_{1} \times \mathrm{Fl}_{\mathcal{D}}
$$

be the trivial bundle map covering $f$ and let $\tau \rightarrow \mathbb{P}\left(A_{1}\right)$ denote the tautological subbundle of $A_{1}$. Then we can define a tautological bundle over $\sigma_{I}^{J}$ by

$$
\rho:=\tilde{f}(\tau)
$$

which is a subbundle of the trivial bundle $A_{1} \subseteq \mathbb{R}^{N}$ over $\sigma_{I}^{J}$.

Note that the intersection $\Omega_{I} \cap B^{-} E_{\bullet}^{J}$ is the Richardson curve minus two points $\sigma_{I}^{J} \backslash\left\{E_{\bullet}^{I}, E_{\bullet}^{J}\right\}$, i.e. $\mathbb{R} P^{1}$ minus two points. We remark that the connected components of this intersection correspond to the pairs offlows in the terminology of Kocherlakota [38]. 
Fig. 1 An illustration of Richardson curves

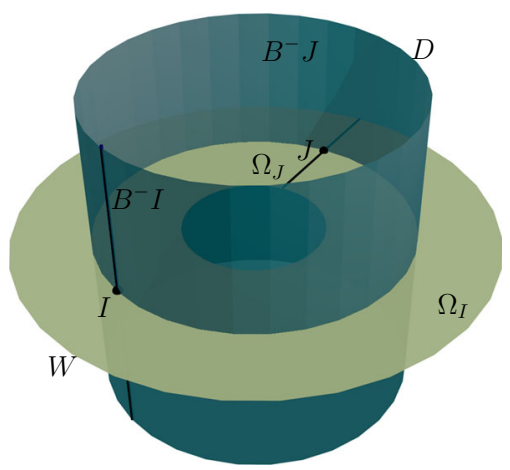

\subsection{Incidence coefficients}

Let $W:=\Omega_{I} \cup \Omega_{J}$ and let $D:=B^{-} E_{\bullet}^{J} \cup B^{-} E_{\bullet}^{I}$ which is a transversal submanifold to $\Omega_{J}$ at $E_{\bullet}^{J}$. Note that $W$ and $D$ are smooth. Indeed, complex Schubert varieties $\sigma_{I}^{\mathbb{C}} \subseteq \mathrm{Fl}_{\mathcal{D}}^{\mathbb{C}}$ are normal [9], so their singularities are of codimension at least 2 and are unions of Schubert cells. Therefore $\Omega_{I}^{\mathbb{C}}$ union the adjacent complex Schubert cells is smooth. This also implies that $W$ and $D$ are smooth in the real case: by Whitney's $\operatorname{Lemma}[58, \operatorname{Lemma} 9] \operatorname{Reg}\left(\sigma_{I}^{\mathbb{R}}\right)=\operatorname{Reg}\left(\sigma_{I}^{\mathbb{C}}\right)(\mathbb{R})$, i.e. the regular points of $\sigma_{I}^{\mathbb{R}}$ are the real points of the regular points of $\sigma_{I}^{\mathbb{C}}$. For an illustration of the notation, see Fig. 1.

The Richardson curve $R:=\sigma_{I}^{J}$ is the transversal intersection of $W$ and $D$ (the intersection of the two surfaces on Fig. 1). Let $R_{+} \cup R_{-}=R \backslash\{I, J\}$ denote the two branches of the Richardson curve (the choice of the sign is arbitrary). Note that $R_{ \pm}$ are the curves denoted by $L_{i}$ in Sect. 2.1. To compute $\left[\Omega_{I}, \Omega_{J}\right]$, we are going to use smoothness of $W$ and $D$.

Proposition 3.1 With the previous notations, the incidence coefficients are determined up to sign by the triviality of the normal bundle $\left.v(W \hookrightarrow X)\right|_{R}$ :

$$
\left[\Omega_{I}, \Omega_{J}\right]= \begin{cases}0 & \text { if }\left.v(W \hookrightarrow X)\right|_{R} \text { is trivial } \\ \pm 2 & \text { if }\left.v(W \hookrightarrow X)\right|_{R} \text { is nontrivial }\end{cases}
$$

Triviality of $\left.\nu(W \hookrightarrow X)\right|_{R}$ is determined by the first Stiefel-Whitney class $w_{1}(\nu(W \hookrightarrow$ $\left.X)\left.\right|_{R}\right)$.

Proof Since $R$ is the transversal intersection of $W$ and $D$, there is a short exact sequence

$$
\left.\left.0 \longrightarrow T R \longrightarrow T D\right|_{R} \longrightarrow v(W)\right|_{R} \longrightarrow 0
$$

where $v(W)$ is the normal bundle of $W$ in $X$. Take a splitting of this short exact sequence:

$$
\left.T D\right|_{R}=T R \oplus N_{W}
$$


For the differentials of the Vassiliev complex, one has to compare the following two orientations for each branch $R_{ \pm}$:

- $\left.T D\right|_{J}$ oriented by the coorientation of $\Omega_{J}$

- $\left.N_{W}\right|_{J}$ oriented by extending the coorientation of $\left.\Omega_{I}\right|_{R_{ \pm}}$to $J$ and $T R$ oriented towards $J$ on both branches $R_{ \pm}$.

To compute $\left[\Omega_{I}, \Omega_{J}\right]$ up to sign, it is enough to compare how the two coorientations $\left.\Omega_{I}\right|_{R_{ \pm}}$extend to $\left.N_{W}\right|_{J}$. This amounts to deciding orientability of the bundle $\left.N_{W} \cong v(W)\right|_{R}$. If $N_{W}$ is orientable, then since its orientation on both branches agrees with its orientation at $I$, the orientations of $\left.N_{W}\right|_{R_{ \pm}}$extend to $J$ identically. Since the orientations of $T R$ induced by the orientations of $T R_{ \pm}$differ at $J$, in this case $\left[\Omega_{I}, \Omega_{J}\right]=0$.

If $N_{W}$ is not orientable, then since the orientations of $\left.N_{W}\right|_{R_{+}}$and $\left.N_{W}\right|_{\bar{R}_{-}}$agree at $I$, they are different at $J$. In this case $\left[\Omega_{I}, \Omega_{J}\right]= \pm 2$. Finally, vector bundles over $S^{1}$ are classified by their first Stiefel-Whitney class $w_{1}$.

In the upcoming Sects. 3.4-3.6 we determine triviality of $\left.v(W)\right|_{R}$ by giving linearly independent line subbundles $\left.\lambda_{c d} \subseteq v(W)\right|_{R}$ spanning it (Theorem 3.5), and counting the nontrivial ones (since $R \cong S^{1}$, each $\lambda_{c d}$ is either a Möbius bundle or a trivial one). Kocherlakota computes the incidence coefficients (up to sign) using a very similar idea: he computes the relative orientations of pairs of flows from $I$ to $J$, which are in our terminology the branches of the Richardson curves.

Remark 3.2 As we have mentioned before, since Schubert varieties are normal, the singularities have codimension at least 2 and the singular part is a union of Schubert cells. Therefore $\Omega_{I}$ union the adjacent cells is smooth. Let us denote this union by $\circ_{I}$. This gives a new stratification of $\sigma_{I}$, with no one-codimensional stratum, but now the strata are no longer contractible. Now $\sigma_{I}$ is a cycle if and only if $\stackrel{\circ}{\sigma}_{I}$ is coorientable. Indeed, by the previous Proposition, this is the information encoded in $\left[\Omega_{I}, \Omega_{J}\right]$ : the normal bundle of $\sigma_{I}^{\circ}$ restricted to the Richardson curve $\sigma_{I}^{J}$ is orientable iff this coefficient vanishes. Then $\stackrel{\circ}{\sigma}_{I}$ is coorientable iff $v\left(\sigma_{I}^{\circ}\right)$ restricted to the Richardson curve $\sigma_{I}^{J}$ is orientable (trivial) for all adjacent $J$.

For general stratified submanifolds, the union with the one-codimensional strata is not smooth, but when it is, this method is sufficient to decide cycleness. However to compute the cohomology groups we need more, namely to determine the incidence coefficients, which cannot be deduced only from orientability.

\subsection{Splitting $\left.T X\right|_{R}$}

To determine triviality of $\left.v(W)\right|_{R}$, we split $\left.T X\right|_{R}$ into line subbundles $\lambda_{c d}$, parametrized by $T_{I} \amalg N_{I}$ (for the notation $T_{I}, N_{I}$, see Proposition 2.3). We will show that $\left.T W\right|_{R}=\bigoplus_{(c, d) \in T_{I}} \lambda_{c d}$, so $\bigoplus_{(c, d) \in N_{I}} \lambda_{c d}$ is isomorphic to $\left.v(W)\right|_{R}$, see Theorem 3.5 . 
In (3.3) we will specify $\lambda_{c d} \rightarrow R$ as subbundles of $\left.T X\right|_{R} \subseteq \operatorname{End}\left(\mathbb{R}^{N}\right)$. In particular each $\lambda_{c d}$ is of the form $\operatorname{Hom}\left(\mu_{1}, \mu_{2}\right)$ :

$$
\mu_{i} \in\left\{\rho, \rho^{\vee}, \varepsilon_{k}: k \in[N]\right\},
$$

where $\rho \rightarrow R$ is the tautological bundle defined in (3.1), and $\rho^{\vee}$ denotes its orthogonal complement.

Recall the quotient bundles introduced in (2.6). A choice of a basis $e_{i} \in \varepsilon_{i}$ induces a scalar product on $\mathbb{R}^{N}$; this realizes the quotient bundles $Q_{i}, D_{i}$ as subbundles of $\mathbb{R}^{N}$. This induces the following splittings over $X=\mathrm{Fl}_{\mathcal{D}}\left(\mathbb{R}^{N}\right)$ :

$$
\mathbb{R}^{N}=S_{i} \oplus Q_{i}=\bigoplus_{j=1}^{m} D_{j}=\bigoplus_{k=1}^{N} \varepsilon_{k}, \quad S_{i}=\bigoplus_{j=1}^{i} D_{j}, \quad Q_{i}=\bigoplus_{j=i+1}^{m} D_{j}
$$

for all $i=1, \ldots, m$. By restricting to the Richardson curve $R=\sigma_{I}^{J}$,

$$
\left.D_{i}\right|_{R}= \begin{cases}\bigoplus_{j \in I_{i}} \varepsilon_{j}, & i \neq \alpha, \beta, \\ \rho \bigoplus_{a \neq j \in I_{\alpha}} \varepsilon_{j}, & i=\alpha \\ \rho^{\vee} \bigoplus_{b \neq j \in I_{\beta}} \varepsilon_{j}, & i=\beta\end{cases}
$$

where $\rho \rightarrow R$ is the tautological bundle defined previously in (3.1). Then via the isomorphism

$$
T X \cong \bigoplus_{i<j} \operatorname{Hom}\left(D_{i}, D_{j}\right) \subseteq \operatorname{End}\left(\mathbb{R}^{N}\right)
$$

the decomposition (3.2) induces a splitting of $\left.T X\right|_{R}$ into line bundles $\lambda_{c d} \rightarrow R$ parametrized by $(c, d) \in T_{I} \amalg N_{I}$, defined as follows

$$
\lambda_{c d}:= \begin{cases}\operatorname{Hom}\left(\rho, \rho^{\vee}\right), & \text { if } c=a, d=b \\ \operatorname{Hom}\left(\rho, \varepsilon_{d}\right), & \text { if } c=a, I(a)<I(d) \leq I(b), d \neq b \\ \operatorname{Hom}\left(\varepsilon_{c}, \rho^{\vee}\right), & \text { if } d=b, I(a) \leq I(c)<I(b), c \neq a \\ \varepsilon_{c d}, & \text { else. }\end{cases}
$$

where in the else line we use that $\operatorname{Hom}\left(\rho \oplus \rho^{\vee}, \varepsilon_{k}\right)=\operatorname{Hom}\left(\varepsilon_{a} \oplus \varepsilon_{b}, \varepsilon_{k}\right)$.

\subsection{The case of $\mathrm{FI}\left(\mathbb{R}^{3}\right)$}

Next, we will show that $\left\{\lambda_{c d}:(c, d) \in T_{I}\right\}$ span $\left.T W\right|_{R}$. We show this by reducing the general case $\mathrm{Fl}_{\mathcal{D}}$ to the flag manifolds $\mathrm{Fl}_{\mathcal{D}^{\prime}}\left(\mathbb{R}^{3}\right)$. In this section we treat this special case: we compute the tangent bundles $\left.T W^{\prime}\right|_{R}$ for all Schubert varieties in $\mathrm{Fl}_{\mathcal{D}^{\prime}}\left(\mathbb{R}^{3}\right)$. Then the general case of $\mathrm{Fl}_{\mathcal{D}}$ can be reduced to these results, by using direct sum maps $f: \mathrm{Fl}_{\mathcal{D}^{\prime}}\left(\mathbb{R}^{3}\right) \hookrightarrow \mathrm{Fl}_{\mathcal{D}}$. This will be used in Sect. 3.6, where we show $\left.\lambda_{c d} \subseteq T W^{\prime}\right|_{R}$ 
Table 1 The bundle $\left.T \sigma_{I}\right|_{R} / T R$, for $R=R_{a b}=\sigma_{I}^{J}$

\begin{tabular}{llll}
\hline$(a, b)$ & $(2,1)$ & $(3,1)$ & $(3,2)$ \\
\hline$\sigma_{321}$ & $\varepsilon_{32} \oplus \varepsilon_{31}$ & $\varepsilon_{32} \oplus \varepsilon_{21}$ & $\varepsilon_{31} \oplus \varepsilon_{21}$ \\
$\sigma_{231}$ & $\operatorname{Hom}\left(\varepsilon_{3}, \rho^{\vee}\right)$ & $\varepsilon_{21}$ & - \\
$\sigma_{312}$ & - & $\varepsilon_{32}$ & $\operatorname{Hom}\left(\rho, \varepsilon_{1}\right)$ \\
\hline
\end{tabular}

by showing that $\lambda_{c d} \subseteq d f\left(T W^{\prime}\right)$ for some smooth submanifold $W^{\prime} \subseteq \mathrm{Fl}_{\mathcal{D}^{\prime}}\left(\mathbb{R}^{3}\right)$ with $f\left(W^{\prime}\right) \subseteq W$.

Let $\mathcal{D}^{\prime}=(1,1,1), \varepsilon_{i}=\left\langle e_{i}\right\rangle$ and $E_{\bullet}$ be the standard flag, $E_{i}=\oplus_{j=1}^{i} \varepsilon_{j}$. Then

- $\sigma_{321}=X$

- $\sigma_{231}=\left\{F_{\bullet}: F_{1} \subseteq E_{2}\right\}$

- $\sigma_{312}=\left\{F_{\bullet}: E_{1} \subseteq F_{2}\right\}$

- $\sigma_{213}=\left\{F_{\bullet}: F_{2}=E_{2}\right\}$

- $\sigma_{132}=\left\{F_{\bullet}: F_{1}=E_{1}\right\}$

- $\sigma_{123}=\left\{F_{\bullet}: F_{1}=E_{1}, F_{2}=E_{2}\right\}$

where we use the one-line notation as discussed after (2.1). All of these Schubert varieties are smooth. The tangent bundles of the $\geq 2$-dimensional Schubert varieties are therefore:

$$
\begin{aligned}
& T \sigma_{231}=\operatorname{Hom}\left(S_{1}, E_{2} / S_{1}\right) \oplus \operatorname{Hom}\left(D_{2}, D_{3}\right), \\
& T \sigma_{312}=\operatorname{Hom}\left(S_{1}, D_{2}\right) \oplus \operatorname{Hom}\left(S_{2} / E_{1}, D_{3}\right), \\
& T \sigma_{321}=T X .
\end{aligned}
$$

Let $I, J \in \operatorname{OSP}(\mathcal{D}), \ell(J)=\ell(I)-1$ and $J$ be obtained by interchanging $a \in I_{\alpha}$ with some $b \in I_{\beta}, a>b, \alpha<\beta$. If $I$ is fixed, denote the Richardson curve $\sigma_{I}^{J}$ by $R_{a b}$. Then by restricting to the Richardson curves $R=R_{a b}$, we obtain the expressions for $\left.T \sigma_{I}\right|_{R} / T R$ described in Table 1 , where $\varepsilon_{i j}=\left.\operatorname{Hom}_{\mathbb{R}}\left(\varepsilon_{i}, \varepsilon_{j}\right) \subseteq T X\right|_{R}$ and $\rho \rightarrow R$ is the tautological bundle as described earlier.

Table 1 shows that $\left\{\lambda_{c d}:(c, d) \in T_{I}\right\}$ defined in (3.3) spans $\left.T W\right|_{R}$.

Similarly, for $\mathcal{D}^{\prime}=(1,2), \mathrm{Fl}_{\mathcal{D}^{\prime}}\left(\mathbb{R}^{3}\right)=\mathbb{P}^{2}$, the only $\geq 2$-dimensional orbit is $I=(3)(1,2), J=(2)(1,3)$ and

$$
\left.T \sigma_{I}\right|_{R} / T R=\operatorname{Hom}\left(\rho, \varepsilon_{1}\right)
$$

In case $\mathcal{D}^{\prime}=(2,1), \mathrm{Fl}_{\mathcal{D}^{\prime}}\left(\mathbb{R}^{3}\right)=\mathrm{Gr}_{2}\left(\mathbb{R}^{3}\right)$, the only $\geq 2$-dimensional orbit is $I=$ $(2,3)(1), J=(1,3)(2)$ and

$$
\left.T \sigma_{I}\right|_{R} / T R=\operatorname{Hom}\left(\varepsilon_{3}, \rho^{\vee}\right)
$$

\subsection{Decomposing TW}

Let us return to the general case $X=\mathrm{Fl}_{\mathcal{D}}^{\mathbb{R}}$, and fix adjacent $I, J, a \in I_{\alpha}, b \in I_{\beta}$, $R=\sigma_{I}^{J}, W=\Omega_{I} \cup \Omega_{J}$ as before. 
In this section we show that

$$
\left.T W\right|_{R}=\bigoplus_{(c, d) \in T_{I}} \lambda_{c d}
$$

for $\lambda_{c d}$ defined in (3.3). We show this by embedding smooth submanifolds $f: W^{\prime} \hookrightarrow$ $W$, such that $\left.\left.\lambda_{c d} \subseteq d f\left(T W^{\prime}\right)\right|_{R} \subseteq T W\right|_{R}$ for all $(c, d) \in T_{I}$. The $W^{\prime}$ are submanifolds of smaller flag manifolds $\mathrm{Fl}_{\mathcal{D}_{1}}$ which are embedded in $\mathrm{Fl}_{\mathcal{D}}\left(\mathbb{R}^{N}\right)$ via the direct sum maps of Sect. 2.2.3.

For each $(c, d) \in T_{I}$ distinct from $(a, b)$ we specify a direct sum map. Set $\vartheta:=$ $\{a, b, c, d\}$ which has 3 or 4 elements, and let

$$
\left(\mathcal{D}_{1}\right)_{\kappa}:=|\{k \in \vartheta: I(k)=\kappa\}|, \quad \kappa=1, \ldots, m
$$

the number of distinct elements in $\vartheta$ which are in $I_{\kappa}$ (this is either 0,1 or 2). Let $\mathcal{D}_{2}:=\mathcal{D}-\mathcal{D}_{1}$ and $\Theta=\left\langle\varepsilon_{k}: k \in \vartheta\right\rangle$. The decomposition $\mathbb{R}^{N}=\Theta \oplus \Theta^{\vee}$ induces the direct sum map

$$
F: \mathrm{Fl}_{\mathcal{D}_{1}}(\Theta) \times \mathrm{Fl}_{\mathcal{D}_{2}}\left(\Theta^{\vee}\right) \rightarrow \mathrm{Fl}_{\mathcal{D}}\left(\mathbb{R}^{N}\right)
$$

Let $E_{\bullet}^{I J}:=E_{\bullet}^{I} \cap E_{\bullet}^{J} \in \mathrm{Fl}_{\mathcal{D}_{2}}\left(\Theta^{\vee}\right)$. Define the embedding $f: \mathrm{Fl}_{\mathcal{D}_{1}}(\Theta) \hookrightarrow$ $\mathrm{Fl}_{\mathcal{D}}\left(\mathbb{R}^{N}\right)$ by $F\left(\cdot, E_{\bullet}^{I J}\right)$.

Proposition 3.3 Given adjacent $I, J \in \operatorname{OSP}(\mathcal{D})$, there exist (unique) $I^{\prime}, J^{\prime} \in\left(\begin{array}{l}|\vartheta| \\ \mathcal{D}_{1}\end{array}\right)$, such that the following diagram commutes:

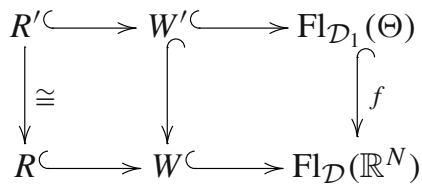

where $R^{\prime}=\sigma_{I^{\prime}}^{J^{\prime}} \subseteq \mathrm{Fl}_{\mathcal{D}_{1}}(\Theta), W^{\prime}=\Omega_{I^{\prime}} \cup \Omega_{J^{\prime}} \subseteq \mathrm{Fl}_{\mathcal{D}_{1}}(\Theta)$ are smooth submanifolds.

Proof Since $f$ is an embedding, $E_{\bullet}^{I}$ and $E_{\bullet}^{J}$ have at most one preimage each. There are unique order preserving maps

$$
n:\{1, \ldots,|\vartheta|\} \rightarrow \vartheta, \quad p:\{1, \ldots,|I(\vartheta)|\} \rightarrow I(\vartheta)
$$

Let the maps

$$
I^{\prime}, J^{\prime}:\{1, \ldots,|\vartheta|\} \rightarrow\{1, \ldots,|I(\vartheta)|\}
$$

be defined by $I^{\prime}(i):=p^{-1}(I(n(i))), J^{\prime}(i):=p^{-1}(J(n(i)))$. Then $f\left(E_{\bullet}^{I^{\prime}}\right)=E_{\bullet}^{I}$ and $f\left(E_{\bullet}^{J^{\prime}}\right)=E_{\bullet}^{J}$. Since $f$ is $\operatorname{GL}(\Theta)$-equivariant, $f\left(R^{\prime}\right)=R$, and $f\left(W^{\prime}\right) \subseteq W$.

Corollary $3.4 d f\left(T W^{\prime}\right) \subseteq T W$ is a subbundle. 
We will use the following theorem to determine triviality of $\left.v(W)\right|_{R}$.

Theorem 3.5 TW $\left.\right|_{R}$ is an inner direct sum of the subbundles $\left\{\lambda_{c d}:(c, d) \in T_{I}\right\}$ where $\lambda_{c d}$ are defined in (3.3). $\left.v(W)\right|_{R}$ is isomorphic to $\bigoplus_{(c, d) \in N_{I}} \lambda_{c d}$.

We split the proof of Theorem 3.5 into several parts. First, we prove Proposition 3.6, whose Corollaries 3.7, 3.8 are exactly the statement of Theorem 3.5.

Proposition $3.6\left\{\lambda_{c d}:(c, d) \in T_{I}\right\}$ are subbundles of $\left.T W\right|_{R}$, where $\lambda_{c d}$ are defined in (3.3).

Proof Let $(c, d) \in T_{I}$ and set $\vartheta=\{a, b, c, d\}$ as above, $\vartheta_{1}=\{a, b\}$ and $\vartheta_{2}=\vartheta \backslash \vartheta_{1}$ (this has either 1 or 2 elements).

If $|\vartheta|=4$, then by Proposition $2.3(c, d) \in T_{J}$. In this case we can further decompose $\mathcal{D}_{1}$ as

$$
\left(\mathcal{D}_{1 i}\right)_{\kappa}=\left|\left\{k \in \vartheta_{i}: I(k)=\kappa\right\}\right|, \quad \kappa=1, \ldots, m
$$

$\mathcal{D}_{1}=\mathcal{D}_{11}+\mathcal{D}_{12}$ and $\Theta=\Theta_{1} \oplus \Theta_{2}$ for $\Theta_{i}=\left\langle\varepsilon_{k}: k \in \vartheta_{i}\right\rangle$. Note that $\mathcal{D}_{1 i}=(1,1)$, $(i=1,2)$ in the appropriate positions. This decomposition induces another direct sum map

$$
g: \mathbb{P} \Theta_{1} \times \mathbb{P} \Theta_{2} \rightarrow \mathrm{Fl}_{\mathcal{D}_{1}}(\Theta)
$$

Let $W^{\prime \prime}:=\mathbb{P} \Theta_{1} \times\left(\mathbb{P} \Theta_{2} \backslash \mathbb{P} \varepsilon_{d}\right)$ and $R^{\prime \prime}:=\mathbb{P} \Theta_{1} \times \mathbb{P} \varepsilon_{c}$. As in the proof of Proposition 3.3, equivariance shows that $W^{\prime}:=\Omega_{I^{\prime}} \cup \Omega_{J^{\prime}}=g\left(W^{\prime \prime}\right)$ and $R^{\prime}=g\left(R^{\prime \prime}\right)$. Then $\left.\varepsilon_{c d} \subseteq T W^{\prime \prime}\right|_{R^{\prime \prime}}$. Applying Proposition 2.4 for $g$ and $f$,

$$
\lambda_{c d}=\left.d(f \circ g) \varepsilon_{c d} \subseteq T W\right|_{R} .
$$

If $|\vartheta|=3$, we are in the case of $\mathcal{D}_{1}=(1,1,1), \mathcal{D}_{1}=(2,1)$ or $\mathcal{D}_{1}=(1,2)$. Then Table 1 shows that the Proposition holds for $I^{\prime}, J^{\prime} \in\left(\begin{array}{c}3 \\ \mathcal{D}_{1}\end{array}\right)$ and $\left(c^{\prime}, d^{\prime}\right) \in T_{I^{\prime}}$. By Proposition 2.4, $\left.\lambda_{c d} \subseteq T W\right|_{R}$.

Corollary 3.7 The subbundles $\left\{\lambda_{c d}:(c, d) \in T_{I}\right\}$ are linearly independent and therefore span $\left.T W\right|_{R}$.

Proof Set $\vartheta_{1}=\{a, b\}$ and $\Theta_{1}=\varepsilon_{a} \oplus \varepsilon_{b}$. It is enough to show that the bundles $\lambda_{c d}$ are linearly independent in each summand

$$
\operatorname{End}\left(\mathbb{R}^{N}\right)=\operatorname{End}\left(\Theta_{1}^{\vee}\right) \oplus \operatorname{End}\left(\Theta_{1}\right) \oplus \bigoplus_{k \neq a, b}\left(\operatorname{Hom}\left(\Theta_{1}, \varepsilon_{k}\right) \oplus \operatorname{Hom}\left(\varepsilon_{k}, \Theta_{1}\right)\right)
$$

Given $c, d$, set $\vartheta=\{a, b, c, d\}, \vartheta_{2}=\vartheta \backslash \vartheta_{1}$, and $\Theta_{2}=\left\langle\varepsilon_{k}: k \in \vartheta_{2}\right\rangle$.

If $|\vartheta|=4$, then $\lambda_{c d}=\varepsilon_{c d}$ which are linearly independent in $\operatorname{End}\left(\Theta_{1}^{\vee}\right)$.

If $|\vartheta|=3$, then $\left|\vartheta_{2}\right|=1$, denote its single element by $k$. Then

$$
\lambda_{c d} \subseteq \operatorname{Hom}\left(\Theta_{1}, \varepsilon_{k}\right) \oplus \operatorname{Hom}\left(\varepsilon_{k}, \Theta_{1}\right)
$$


For fixed $k$ there are at most $2 \operatorname{such}(c, d)$ pairs, since $c>d$ for $(c, d) \in T_{I}$. So it is enough to check linear independence of such pairs. dent.

If $a>k>b$, then $\lambda_{a k} \subseteq \operatorname{Hom}\left(\Theta_{1}, \varepsilon_{k}\right), \lambda_{k b} \subseteq \operatorname{Hom}\left(\varepsilon_{k}, \Theta_{1}\right)$, so they are indepen-

If $a>b>k$ then in order for $(a, k),(b, k) \in T_{I}$ to hold, $I(a)<I(b)<I(k)$ must hold, then $\lambda_{a k}=\varepsilon_{a k}, \lambda_{b k}=\varepsilon_{b k}$ are independent in $\operatorname{Hom}\left(\Theta_{1}, \varepsilon_{k}\right)$. The case $k>a>b$ is similar.

Finally, $\lambda_{a b}=\operatorname{Hom}\left(\rho, \rho^{\vee}\right) \subseteq \operatorname{End}\left(\Theta_{1}\right)$.

Corollary $\left.3.8 v(W)\right|_{R}$ is isomorphic to $\bigoplus_{(c, d) \in N_{I}} \lambda_{c d}$.

This concludes the proof of Theorem 3.5. Let us introduce some notation.

Definition 3.9 Fix $I \in \operatorname{OSP}(\mathcal{D}), \mathcal{D}=\left(d_{1}, \ldots, d_{m}\right)$, and fix $a, b \in\{1, \ldots, N\}$ such that $\alpha=I(a), \beta=I(b), \alpha<\beta$. For $c \in\{1, \ldots, N\}, \gamma, \delta \in\{0,1, \ldots, m\}$, set

$$
\begin{aligned}
G_{I}(c, \gamma, \delta) & :=|\{d>c: \gamma<I(d) \leq \delta\}|, \\
L_{I}(c, \gamma, \delta) & :=|\{d<c: \gamma<I(d) \leq \delta\}| \\
T_{I}(a, b) & :=L_{I}(a, \alpha, \beta)+G_{I}(b, \alpha-1, \beta-1), \\
N_{I}(a, b) & :=G_{I}(a, \alpha, \beta)+L_{I}(b, \alpha-1, \beta-1) \\
G_{I}(a, \delta) & :=G_{I}(a, \delta, m), \quad G_{I}(a):=G_{I}(a, \alpha),
\end{aligned}
$$

abbreviating Greater, Less, Tangent, Normal.

We will be interested in these numbers for the fixed adjacent $J \leq I \in \operatorname{OSP}(\mathcal{D})$, where $J$ is obtained by interchanging $a \in I_{\alpha}$ with some $b \in I_{\beta}, a>b, \alpha<\beta$. Note that by Definition (3.3) of $\lambda_{c d}, N_{I}(a, b)$ is the number of nontrivial $\lambda_{c d}$ for $(c, d) \in N_{I}$. Then by Proposition 3.1 and Theorem 3.5, we have:

Theorem 3.10 If $I, J \in \operatorname{OSP}(\mathcal{D}), \ell(J)=\ell(I)-1$ and $J$ is obtained from I by interchanging $a \in I_{\alpha}$ with some $b \in I_{\beta}, a>b, \alpha<\beta$, then using the notations (3.5):

$$
\left[\Omega_{I}, \Omega_{J}\right]= \begin{cases}0, & \text { if } N_{I}(a, b) \text { is even } \\ \pm 2, & \text { if } N_{I}(a, b) \text { is odd }\end{cases}
$$

Example 3.11 Let us compute the incidence coefficient $\left[\Omega_{I}, \Omega_{J}\right]$ up to sign for

$$
I=[36,14,25], \quad J=[26,14,35]
$$

in $\mathrm{Fl}_{2,2,2}$ (recall the notation introduced after (2.1)). In this case $a=3, \alpha=1$, $b=2, \beta=3$. There are two elements in $I_{2} \cup I_{3}$ (4 and 5) greater than $a=3$, so $G_{I}(a, \alpha, \beta)=2$. There is one element in $I_{1} \cup I_{2}$ which is less than $b=2$, so $L_{I}(a, \alpha, \beta)=1$, thus $N_{I}(a, b)=3$ and $\left[\Omega_{I}, \Omega_{J}\right]= \pm 2$.

To any cochain complex, one can define an incidence graph as was done in [12]: the vertices of the graph are elements of $\left(\begin{array}{l}N \\ \mathcal{D}\end{array}\right)$ and $(I, J)$ is an edge iff $\left[\Omega_{I}, \Omega_{J}\right] \neq 0$. See Fig. 3 for the incidence graph of $\mathrm{Fl}\left(\mathbb{R}^{4}\right)$ at the end of this section (the colors correspond to the signs). 
Fig. 2 The double of a Young diagram

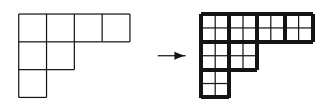

\subsection{Determining the cycles}

In the even case $\mathrm{Fl}_{2 \mathcal{D}}^{\mathbb{R}}$, equation (3.6) is actually sufficient to determine the rational coefficient cohomology $H^{*}\left(\mathrm{Fl}_{2 \mathcal{D}}^{\mathbb{R}} ; \mathbb{Q}\right)$ additively in terms of Schubert cycles, i.e. the sign of \pm 2 can be ignored. For the $\mathbb{Z}$-coefficient cohomology, the signs are required as well, see Sect. 3.9.

If $2 \mathcal{D}=\left(2 d_{1}, 2 d_{2}, \ldots, 2 d_{r}\right)$ and $I \in \operatorname{OSP}(\mathcal{D})$, then the doubled ordered set partition $D I \in \mathrm{OSP}(2 \mathcal{D})$ is obtained by replacing each $i \in I_{j}$ by $(2 i-1,2 i) \in D I_{j}$; each element $k \in D I_{j}$ has a unique pair $k^{\prime} \in D I_{j}$. A double Schubert variety $\sigma_{D I}^{\mathbb{R}} \subseteq \mathrm{Fl}_{2 \mathcal{D}}^{\mathbb{R}}$ is a Schubert variety corresponding to $D I \in \operatorname{OSP}(2 \mathcal{D})$.

In the case of Grassmannians $\mathcal{D}=(k, l), D I \in\left(\begin{array}{c}2(k+l) \\ 2 k\end{array}\right)$ we recover the double Young diagrams of Pontryagin [44], see also [23, III.3.2.2.B] and [20, Theorem 3.3.1]. These Young diagrams are obtained by subdividing each square into $2 \times 2$ squares in the Young diagram corresponding to $I \in\left(\begin{array}{c}k+l \\ k\end{array}\right)$, see Fig. 2. In terms of ordered set partitions, for $\mathcal{D}=(1,1)$, the doubled ordered set partitions are $[12,34]$ and $[34,12] \in \operatorname{OSP}(2 \mathcal{D})$, which are the doubles of $[1,2]$ and $[2,1] \in \operatorname{OSP}(\mathcal{D})$ respectively.

Theorem 3.12 In $\mathrm{Fl}_{2 \mathcal{D}}\left(\mathbb{R}^{N}\right)$ the double Schubert varieties $\sigma_{D I}$ are (integer) cycles and their classes $\left[\sigma_{D I}\right]$ generate a free $\mathbb{Z}$-submodule of $H^{*}\left(\mathrm{Fl}_{2 \mathcal{D}}^{\mathbb{R}} ; \mathbb{Z}\right)$. Rationally, $\left[\sigma_{D I}\right]$ form a basis of $H^{*}\left(\mathrm{Fl}_{2 D}^{\mathbb{R}} ; \mathbb{Q}\right)$.

Proof Let $I=D I^{\prime} \in \operatorname{OSP}(2 \mathcal{D})$ be a doubled ordered set partition. As above, if $\Omega_{J} \subseteq \sigma_{I}$ and $\ell(I)-\ell(J)=1$, then $J$ is obtained by interchanging $a \in I_{\alpha}$ with some $b \in I_{\beta}, \alpha<\beta, a>b$. Since $I$ is doubled, both terms in the sum

$$
N_{I}(a, b)=G_{I}(a, \alpha, \beta)+L_{I}(b, \alpha-1, \beta-1)
$$

are even; e.g. if $k>a$ and $I(k)>\alpha$, then its pair $k^{\prime}$ also satisfies $k^{\prime}>a$ and $I\left(k^{\prime}\right)=I(k)>\alpha$. So all coefficients $\left[\Omega_{I}, \Omega_{J}\right]$ vanish and $\sigma_{I}$ is a cycle.

Now assume that $\Omega_{I} \subseteq \sigma_{J}$ and $\ell(J)-\ell(I)=1$, and $I=D I^{\prime}$ be obtained by interchanging $a \in J_{\alpha}$ with some $b \in J_{\beta}, \alpha<\beta, a>b$. We again have to determine the parity of (3.7), but now for $N_{J}(a, b)$. Let $a^{\prime}$ and $b^{\prime}$ denote the pairs of $a$ and $b$ respectively. Since $I$ is a doubled ordered set partition, $a^{\prime} \in I_{\beta}$ and $b^{\prime} \in I_{\alpha}$. $\ell(J)-\ell(I)=1$ implies that $a<a^{\prime}$ and $b^{\prime}<b$. As before, everything in $J$ appears in pairs, except $a<a^{\prime}$ and $b<b^{\prime}$ which shows that $G_{J}(a, \alpha, \beta)$ and $L_{J}(a, \alpha-1, \beta-1)$ are both odd. So $N_{J}(a, b)$ is even and $\Omega_{I}$ appears in all incidence relations with zero coefficient $\left[\Omega_{J}, \Omega_{I}\right]=0$. Therefore $\left[\sigma_{I}\right]$ does not appear in any relation and the double Schubert cycles $\left\{\left[\sigma_{D J}\right]: J \in \operatorname{OSP}(\mathcal{D})\right\}$ are linearly independent.

Finally, $\operatorname{dim}_{\mathbb{Q}} H^{*}\left(\mathrm{Fl}_{2 \mathcal{D}}^{\mathbb{R}} ; \mathbb{Q}\right)=|\operatorname{OSP}(\mathcal{D})|$, which follows e.g. from Theorem A.3. This agrees with the number of doubled ordered set partitions of $2 \mathcal{D}$. 


\subsection{Kocherlakota's theorem}

Theorem 3.10 gives an alternate proof of Kocherlakota's theorem [38, Theorem A] for the special case of the classical real flag manifolds $\mathrm{Fl}_{\mathcal{D}}^{\mathbb{R}}$. Before stating it we have to introduce some further notation.

Let $\mathfrak{g}$ be a real split semisimple Lie algebra. Let $\mathfrak{a}$ be a maximal $\mathbb{R}$-diagonalizable subalgebra and let $\Sigma \subseteq \mathfrak{a}^{*}$ be the restricted root system. Since $\mathfrak{g}$ is split, all root multiplicities are one. Choose a regular element $\xi \in \mathfrak{a}$, which determines a positive Weyl chamber $C^{+}$and $\Sigma=\Sigma^{+} \bigsqcup \Sigma^{-}$. The reflections $r_{\varphi}$ in the root planes $\operatorname{ker} \varphi$, $\varphi \in \Sigma^{+}$generate the Weyl group $W$ of the root system. The Weyl group acts freely and transitively on the Weyl chambers, and the Weyl chambers $C_{w}$ are labeled by $w \in W, C^{+}=C_{1}$ for $1 \in W$. Given $H \in \overline{C^{+}}$, let $\Theta \subseteq \Sigma^{s}$ be the set of simple roots vanishing at $H$. Then the Weyl orbit $W . H=W / W_{H}$ parametrizes the Bruhat cells of $G / P_{\Theta}$ (cf. [15]). Now we state Kocherlakota's theorem. Given $x \in \mathfrak{a}$, let

$$
\begin{aligned}
\mathcal{N}(x) & :=\left\{\varphi \in \Sigma^{+}: \varphi(x)<0\right\}, \\
\sigma(x) & :=\sum_{\varphi \in N(x)} \varphi \in \mathfrak{a}^{*},
\end{aligned}
$$

and $\ell(x):=|\mathcal{N}(x)|$ (we change the notation of Kocherlakota to $\mathcal{N}$ in order to distinguish from $N_{I}$ ). This is consistent with notation (2.4), as we will show below, and in general it is the dimension of $\Omega_{x} \subseteq G / P_{\Theta}$ for $x \in W . H$. We will give another interpretation of $N(x)$, see (3.8). Let us now recall the theorem of Kocherlakota.

Theorem 3.13 (Kocherlakota) Let $x, y \in W . H=W / W_{H}$ and $\ell(y)=\ell(x)-1$. If $r_{\varphi}(x)=y$ for a reflection $r_{\varphi}, \varphi \in \Sigma^{+}$, then $\sigma(x)-\sigma(y)=m \varphi$ for some $m \in \mathbb{Z}$. The incidence coefficients of the Bruhat cells $\Omega_{x}, \Omega_{y}$ are given by

$$
\left[\Omega_{x}, \Omega_{y}\right]= \begin{cases}0, & m \text { odd } \\ \pm 2, & \text { m even }\end{cases}
$$

Before giving the proof for $\mathfrak{g}=\mathfrak{s l}(N, \mathbb{R})$, let us recall some specifics about the root system of type $A_{N-1}$. The roots in an appropriate basis are $\pm e_{i j}$ where $e_{i j}=e_{i}-e_{j}$, $i<j$. The simple roots are $\delta_{i}=e_{i, i+1}$, and in terms of the simple roots $e_{i j}=\sum_{k=i}^{j} \delta_{i}$. Its Weyl group is $W \cong S_{N}$ and the reflections $r_{i j}$ through the hyperplane $\operatorname{ker} e_{i j}$ correspond to the transpositions $(i j) \in S_{N}$.

The Weyl-orbit of a regular element $H \in C^{+}$can be parametrized by $W \cong S_{N}$. If $H \in \overline{C^{+}}$is not regular, list the simple roots $\delta_{i}$ not vanishing on $H: \delta_{s_{1}}, \delta_{s_{2}}, \ldots, \delta_{s_{r}}$, such that $s_{1}<s_{2}<\ldots s_{r}$, and set $s_{r+1}:=N$. Then the Weyl-orbit $W . H=W / W_{H}=$ $\operatorname{OSP}(\mathcal{D})$, where $\mathcal{D}=\left(d_{1}, \ldots, d_{r}\right)$, for $d_{i}=s_{i+1}-s_{i}$.

This implies that positive roots $e_{i j} \in \Sigma^{+}$have the following property: given $I \in$ $W / W_{H}, e_{i j}(I)<0$ iff $(i, j)$ is an inversion of $I \in W / W_{H}$. Thus $\mathcal{N}(I)$ is the set of inversions of $I \in W / W_{H}$ :

$$
\mathcal{N}(I)=\left\{e_{i j}:(i, j) \text { is an inversion of } I\right\}
$$


In particular, for $I \in \operatorname{OSP}(\mathcal{D}),|\mathcal{N}(I)|=\ell(I)=\operatorname{dim}_{\mathbb{R}} \Omega_{I}$ as we have stated above (e.g. by Proposition 2.3).

Proof (Proof of Theorem 3.13 for $\mathfrak{g}=\mathfrak{s l}(N, \mathbb{R})$ ) Let $I, J \in W / W_{H}=\operatorname{OSP}(\mathcal{D})$, such that $r_{a b}(I)=J$, and $\ell(J)=\ell(I)-1, a>b, a \in I_{\alpha}, b \in I_{\beta}$. By (3.8), the set theoretic difference of $\mathcal{N}(J) \backslash \mathcal{N}(I)$ consists of those $e_{i j}$, for which $i, j$ is an inversion in $J$, but not in $I$.

Clearly all such $i, j$ pairs must contain $a$ or $b$. A simple verification shows that there are three types of elements in $\mathcal{N}(I) \backslash \mathcal{N}(J)$ (the other cases can be excluded using $\ell(J)=\ell(I)-1)$ :

- If $e_{b j} \in \mathcal{N}(I) \backslash \mathcal{N}(J)$ and $a<j$, then $e_{a j} \in \mathcal{N}(J) \backslash \mathcal{N}(I)$,

- if $e_{j a} \in \mathcal{N}(I) \backslash \mathcal{N}(J)$ and $j<b$, then $e_{j b} \in \mathcal{N}(J) \backslash \mathcal{N}(I)$, and

- $e_{b a} \in \mathcal{N}(I) \backslash \mathcal{N}(J)$.

Since $e_{b j}-e_{a j}=e_{b a}$ for $b<a<j$ and $e_{j a}-e_{j b}=e_{b a}$ for $j<b<a$

$$
\begin{aligned}
\sigma(I)-\sigma(J) & =\sum_{e_{i j} \in \mathcal{N}(I) \backslash \mathcal{N}(J)} e_{i j}-\sum_{e_{i j} \in \mathcal{N}(J) \backslash \mathcal{N}(I)} e_{i j} \\
& =\left(G_{I}(a, \alpha, \beta)+L_{I}(b, \alpha-1, \beta-1)+1\right) e_{b a}=\left(N_{I}(a, b)+1\right) e_{b a}
\end{aligned}
$$

using the definitions preceding Theorem 3.10. We can conclude by Theorem 3.10.

\subsection{Signs}

For cooriented $\Omega_{I}$ and $\Omega_{J}$, determining the actual signs of $\left[\Omega_{I}, \Omega_{J}\right]$ requires some further work. We conclude this section by determining the signs. We obtain similar results as [46] who deal with the general case of $R$-spaces. One can make several choices of orientations - we coorient all $\Omega_{I}$ lexicographically as described in Section 3.1 and compute the signs of $\left[\Omega_{I}, \Omega_{J}\right]$ relative to these orientations.

\subsubsection{Geometry}

Before stating the following Proposition describing the signs, let us introduce some notation. Let $I, J \in \operatorname{OSP}(\mathcal{D})$ be adjacent, obtained by swapping $a \in I_{\alpha}$ with some $b \in I_{\beta}$. We will denote by $\left(e_{c} \mapsto e_{d}\right) \in \operatorname{Hom}\left(\varepsilon_{c}, \varepsilon_{d}\right)$ the homomorphism mapping $e_{c}$ to $e_{d}$. Let $R_{+}$be a branch of the Richardson curve $R$ and let $U$ be a slight enlargement of $R_{+}$: a (contractible) connected open set $U \subsetneq R$ containing $\overline{R_{+}}$. Let $r \in \Gamma\left(\left.\rho\right|_{U}\right)$ and $r^{\vee} \in \Gamma\left(\left.\rho^{\vee}\right|_{U}\right)$ be nowhere vanishing sections, such that $r(I)=-r^{\vee}(J)=e_{a}$ and $r^{\vee}(I)=r(J)=e_{b}$ (this choice determines the branch $R_{+}$). Define sections of $\left.\lambda_{c d}\right|_{U}$, for $(c, d) \in N_{I}$ as follows:

$$
s_{c d}:= \begin{cases}\left(e_{c} \mapsto e_{d}\right), & \text { if }(c, d) \in N_{J} \\ \left(r \mapsto e_{d}\right), & \text { if } c=a, I(a)<I(d) \leq I(b) \\ \left(e_{c} \mapsto r^{\vee}\right), & \text { if } d=b, I(a) \leq I(c)<I(b)\end{cases}
$$


These are the only cases that appear in (3.3) for $(c, d) \in N_{I}$, since $(a, b) \in T_{I}$. We will say that $s_{c d}$ is trivial if $s_{c d}=\left(e_{c} \mapsto e_{d}\right)$ and nontrivial otherwise. Notice that $s_{c d}$ is trivial iff $(c, d) \in N_{I}$ and $(c, d) \in N_{J}$.

Proposition 3.14 The sign of $\left[\Omega_{I}, \Omega_{J}\right]$ is +1 iff the following two orientations agree, -1 otherwise:

$N_{J}^{1}:=\left(\left(e_{c} \mapsto e_{d}\right):(c, d) \in N_{J}\right), \quad N_{J}^{2}:=\left(-\left(e_{b} \mapsto e_{a}\right), s_{c d}(J):(c, d) \in N_{I}\right)$

where in both $N_{J}^{1}$ and $N_{J}^{2}$, the terms involving $(c, d)$ are listed lexicographically.

Proof Recall that the incidence coefficient $\left[\Omega_{I}, \Omega_{J}\right]$ in the Vassiliev complex can be computed as a sum of \pm 1 contributions for each branch $R_{ \pm}$of the Richardson curve (cf. Sect. 2.1). The contribution of one of the branches $R_{+}$can be computed as follows. Take the splitting

$$
\left.T B^{-} J\right|_{R_{+}}=\left.T R \oplus v_{I}\right|_{R_{+}},
$$

where $v_{I}$ is the sum of line bundles of Theorem 3.5. Then the contribution of $R_{+}$is obtained by comparing the following two orientations of $T_{J} B_{J}^{-}$:

- the orientation induced by the coorientation of $\left.v\left(\Omega_{J}\right)\right|_{J}$ : this is the lexicographical orientation $N_{J}^{1}=\left(\left(e_{c} \mapsto e_{d}\right):(c, d) \in N_{J}\right)$ and

- the orientation $O_{2}$ determined by the splitting (3.9): $\left.T R\right|_{R_{+}}$is oriented towards $J$, and $\left.v_{I}\right|_{R_{+}}$is oriented by the coorientation of $\Omega_{I}$.

Note that the second orientation has to be extended to $J$. In order to extend the orientation of $\left.T B^{-} J\right|_{R_{+}}$to $J$, we will use the sections $s_{c d}$ : if the orientation $\left(s_{c d}(I)\right)$ agrees with the coorientation $\Omega_{I}$, then the orientation $\left(\left.T R\right|_{U}, s_{c d}(J)\right)$ determines the orientation $\mathrm{O}_{2}$. On the branch $R_{+},\left(r \mapsto r^{\vee}\right)$ points towards $J$, so $\mathrm{O}_{2}$ is exactly $N_{J}^{2}$.

Therefore the combinatorial task is to determine the (relative) sign of two signed permutations.

\subsubsection{Combinatorics}

Before giving a notation heavy answer, let us illustrate on a simple example the computation of the signs:

Example 3.15 Let $\mathcal{D}=\left(1^{6}\right), I=(4,5,6,1,2,3)$ and $J=(4,2,6,1,5,3)$, so $a=5$, $b=2, \alpha=2, \beta=5$. The normal spaces in lexicographical ordering are spanned by

$$
N_{I}=(12)(13)(23)(45)(46)(56), \quad N_{J}=(13)(15)(23)(25)(26)(45)(46)
$$

where $(i j)$ denotes $\pm\left(e_{i} \mapsto e_{j}\right)$ (recall the description of the normal spaces in Proposition 2.3). One has to compare two orientations of $N_{J} \Omega_{J}$, the first orientation being the lexicographical orientation $N_{J}^{1}$. The second orientation is given by 
$N_{J}^{2}=\left((b a), N_{I}(a \leftrightarrow b)\right)$, where $a \leftrightarrow b$ is the operation of exchanging $a$ and $b$ in (cd) $(\{c, d\} \cap\{a, b\} \neq \varnothing)$ if $s_{c d}$ is a nontrivial section (i.e. if (cd) appears only in $\left.N_{I}\right)$. These orientations here are (notice that $(2,3)$ appears in both $N_{I}$ and $N_{J}$, so $s_{23}$ is trivial and there is no substitution $2 \leftrightarrow 5$ for (23)):

$$
N_{J}^{1}=(13)(15)(23)(25)(26)(45)(46), \quad N_{J}^{2}=(25)(15)(13)(23)(45)(46)(26) .
$$

The first difference $c_{1}$ comes from listing $(a b)=(25)$ first in $N_{J}^{2}$, this contributes $c_{1}=3$ transpositions: it precedes (13)(15)(23). The second difference $c_{2}$ comes from exchanging $(a, d)$ with $(b, d)$ if $(a, d) \notin N_{J}$; this contributes $c_{2}=2$ as (26) succeeds (45)(46). The third difference $c_{3}$ comes from exchanging all $(c, b)$ with $(c, a)$ if $(c, b) \notin N_{J}$; this contributes $c_{3}=1$ transpositions; (15) precedes (13). Finally, the nontrivial sections involving $r^{\vee}(J)=-e_{5}$ obtain a sign: this contributes $c_{4}=2$ sign changes, $-\left.\left(e_{1} \mapsto e_{5}\right) \in \operatorname{Hom}\left(e_{1}, \rho^{\vee}\right)\right|_{J}$ and $-\left(e_{2} \mapsto e_{5}\right)$. So the incidence coefficient is $[I, J]=(-1)^{8} 2=+2$, where $c_{1}+c_{2}+c_{3}+c_{4}=8$.

The following Theorem formalizes this computation:

Theorem 3.16 If $\left[\Omega_{I}, \Omega_{J}\right] \neq 0$, then its sign is given by $\left[\Omega_{I}, \Omega_{J}\right]=(-1)^{s(I, J)} \cdot 2$, where

$$
s(I, J)=c_{1}+c_{2}+c_{3}+c_{4}
$$

and using the notations of Definition 3.9, the $c_{i}$ are defined as follows:

$$
\begin{aligned}
c_{1} & =G_{I}(b, \alpha)-G_{I}(a)+\sum_{c<b} G_{I}(c) \\
c_{2} & =\left(G_{I}(a)-G_{I}(a, \beta)\right)\left(\sum_{b<c<a} G_{I}(c)+G_{I}(a, \beta)\right) \\
c_{3} & =\sum_{\substack{c<b \\
\alpha \leq I(c)<\beta}} G_{I}(b, I(c))-G_{I}(a, I(c)) \\
c_{4} & =L_{I}(b, \alpha-1, \beta-1)+1
\end{aligned}
$$

Proof The first permutation is the elements of $N_{J}^{1}$ listed lexicographically:

$$
\begin{aligned}
& \ldots,\left(b-1, d_{n_{b-1}}^{b-1}\right),\left(b, d_{1}^{b}\right), \ldots,(b, a), \ldots,\left(b, d_{n_{b}}^{b}\right), \ldots,\left(c, d_{1}^{c}\right), \ldots,\left(c, d_{n_{c}}^{c}\right) \\
& \quad \ldots,\left(a, d_{1}^{a}\right), \ldots,\left(a, d_{n_{a}}^{a}\right), \ldots
\end{aligned}
$$

The second permutation is obtained by listing $(b, a)$ and then the elements of $N_{I}$ lexicographically

$$
\begin{aligned}
& (b, a), \ldots,\left(b-1, f_{m_{b-1}}^{b-1}\right),\left(b, f_{1}^{b}\right), \ldots,\left(b, f_{m_{b}}^{b}\right) \\
& \quad, \ldots,\left(c, f_{1}^{c}\right), \ldots,\left(c, f_{m_{c}}^{c}\right), \ldots,\left(a, f_{1}^{a}\right), \ldots,\left(a, f_{m_{a}}^{a}\right), \ldots
\end{aligned}
$$


and making the following substitutions, which compared to $N_{J}^{1}$ contribute a certain number of transpositions that we will determine below:

- listing $(b, a)$ first: this contributes $c_{1}$ many transpositions,

- replacing $(a, d)$ with $(b, d)$ for all nontrivial sections $s_{a d}: c_{2}$ many transpositions,

- replacing $(c, b)$ with $(c, a)$ for all nontrivial sections $s_{c d}: c_{3}$ many transpositions.

One must also count the sign differences coming from the values of $s_{c d}(J)=$ $\pm\left(e_{c} \mapsto e_{d}\right)$. These sign differences are represented by the term $c_{4}$. Now we determine $c_{1}, c_{2}, c_{3}, c_{4}$.

The first difference is that $\left(e_{b} \mapsto e_{a}\right)$ is the first element in $N_{J}^{2}$. This contributes

$$
c_{1}=|\{b<c<a: J(b)<J(c)\}|+\sum_{c<b} G_{I}(c)=G_{I}(b, \alpha)-G_{I}(a)+\sum_{c<b} G_{I}(c)
$$

many transpositions.

Given a nontrivial section $s_{a d},(a, d) \in N_{I}: s_{a d}(J)= \pm\left(e_{b} \mapsto e_{d}\right)$, it has the following distance from its final position at $(b, d)$ in $N_{J}^{1}:(a>b)$

$$
\begin{aligned}
& |\{a<c<d: I(b)<I(c)\}|+|\{d<c: I(b)<I(c)\}|+\sum_{b<c<a} G_{I}(c)= \\
& \quad=G_{I}(a, \beta)-G_{I}(d, \beta)+G_{I}(d, \beta)+\sum_{b<c<a} G_{I}(c)
\end{aligned}
$$

as $\left(e_{a} \mapsto e_{d}\right)$ swaps place with every $\left(e_{f} \mapsto e_{g}\right)$ pair whose position doesn't change and precedes it. The sum of these for nontrivial $s_{a d}$ pairs is the term $c_{2}$.

Similarly, for a nontrivial section $s_{c b},(c, b) \in N_{I}, s_{c b}(J)= \pm\left(e_{c} \mapsto e_{a}\right)$ has the following distance from its final position:

$$
|\{b<d<a: I(c)<I(d)\}|=G_{I}(b, I(c))-G_{I}(a, I(c))
$$

the sum of which for nontrivial $s_{c b}$ pairs is $c_{3}$.

Finally, the sections $s_{c d}$ induce $L_{I}(b, \alpha-1, \beta-1)+1$ many sign changes: the trivial bundles have trivial sections, the nontrivial sections $s_{c d}$ involving only $r$ introduce no sign change, whereas each bundle involving $r^{\vee}(J)=-e_{a}$ contributes a sign change, the number of which is $c_{4}=L_{I}(b, \alpha-1, \beta-1)+1$.

Remark 3.17 The sign of $\left[\Omega_{I}, \Omega_{J}\right]$ determines a coloring of the incidence graph (see Fig. 3). Taking the opposite orientation of a vertex $I$, changes the color of all edges incident to $I$, however since in the end such a graph computes the cohomology of $\mathrm{Fl}_{\mathcal{D}}$, the cohomology of the chain complex is the same.

For another choice of orientations, see [46]. There a reduced decomposition $w=r_{1} \cdot \ldots \cdot r_{d}$ is fixed for each $w \in S_{N}$, and such a reduced decomposition determines an ordering of the inversions of $w$, i.e. an orientation of $T_{I}$. Such an ordering is convenient, since for adjacent $w^{\prime}<w=r_{1} \cdot \ldots \cdot r_{d}$, $w^{\prime}$ has a reduced decomposition obtained by omitting some uniquely defined $r_{i}$ - then the relative orientation 


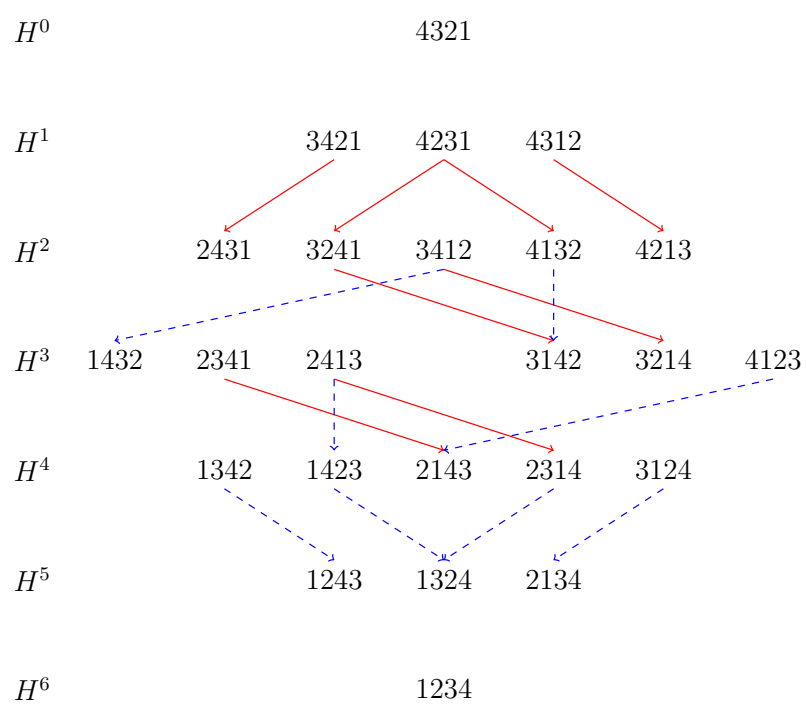

Fig. 3 The signed incidence graph of $\mathrm{Fl}_{4}$ : blue (dashed) edges signify +2 , red (solid) edges -2

of $r_{i}, r_{1}, \ldots, \hat{r_{i}}, \ldots, r_{d}$ and $r_{1}, \ldots, r_{d}$ is simply $(-1)^{i}$. However, the initially fixed reduced decomposition of $w^{\prime}$ might differ from $r_{1} \cdot \ldots \cdot \hat{r}_{i} \cdot \ldots \cdot r_{d}$, so one also has a term comparing these two orientations.

The signs obtained in Theorem 3.16 can be implemented in a computer program, and can be used to compute the cohomology groups of real flag manifolds with integer coefficients. We were mainly interested in the Schubert cycle generators of rational coefficient cohomology. We used SageMath's homology package [52]. See Appendix $\mathrm{B}$ for results in some cases not covered by Theorem 3.12.

\subsection{An example $\mathrm{FI}\left(\mathbb{R}^{4}\right)$}

Figure 3 contains the incidence graph of $\mathrm{Fl}\left(\mathbb{R}^{4}\right)$ defined as follows. The vertices of the graph are the Schubert cells $X_{\alpha}, \alpha \in S_{4}$ and two vertices $X_{\alpha}, X_{\beta}$ are connected by an edge if $\left[X_{\alpha}, X_{\beta}\right]= \pm 2$. This diagram can also be found in [12, p. 529]. The extra information is the coloring of the graph representing the signs: a blue (dashed) edge represents $\left[\Omega_{I}, \Omega_{J}\right]=+2$ a red (solid) edge corresponds to -2 . In this case, in order to compute the cohomology, the signs are actually not needed, as can be seen from the form of the graph; one can read off the cohomology groups of $\mathrm{Fl}\left(\mathbb{R}^{4}\right)$ :

$$
\begin{aligned}
& H^{0}=\mathbb{Z}, \quad H^{1}=0, \quad H^{2}=\mathbb{Z}_{2}^{\oplus 3}, \quad H^{3}=\mathbb{Z}^{\oplus 2} \oplus \mathbb{Z}_{2}^{\oplus 2}, \quad H^{4}=\mathbb{Z}_{2}^{\oplus 2}, \\
& H^{5}=\mathbb{Z}_{2}^{\oplus 3}, \quad H^{6}=\mathbb{Z}
\end{aligned}
$$

The generators of the cohomology groups are not necessarily unique: $x=1432$ and $y=3214$ generate a submodule isomorphic to $\mathbb{Z} \oplus \mathbb{Z}_{2}$ - their sum is a 2-torsion element, whereas either of them generates a free $\mathbb{Z}$-submodule. The smallest instance 
where we encountered a possible dependence on the choice of the signs is $\operatorname{Fl}\left(\mathbb{R}^{6}\right)$ : by changing the signs so that the incidence graph still defines a chain complex, the cohomology groups can be different. Therefore the signs are indeed required in certain cases.

\section{The ring structure of $H^{*}\left(\mathrm{FI}_{2 \mathcal{D}}^{\mathbb{R}} ; \mathbb{Q}\right)$ : Schubert calculus}

The double Schubert varieties $\sigma_{D I}^{\mathbb{R}}$ are cycles and their classes form a basis of $H^{*}\left(\mathrm{Fl}_{2 \mathcal{D}}^{\mathbb{R}} ; \mathbb{Q}\right)$ by Theorem 3.12. By showing that $\mathrm{Fl}_{2 \mathcal{D}}^{\mathbb{R}}$ are circle spaces $[17,41]$ we can deduce their structure constants by relating them to the structure constants of Schubert cycles in complex flag manifolds. Our main tool is Theorem 4.2, the generalized Borel-Haefliger theorem, [17]. Applying this theorem, we obtain that any formula involving Schubert cycles $\left[\sigma_{I}^{\mathbb{C}}\right]$ in a complex partial flag manifold $\mathrm{Fl}_{\mathcal{D}}^{\mathbb{C}}$, holds for the doubled real Schubert cycles $\left[\sigma_{D I}^{\mathbb{R}}\right]$ in the double real partial flag manifold $\mathrm{Fl}_{2 \mathcal{D}}^{\mathbb{R}}$.

Circle spaces are analogues of conjugation spaces introduced by Hausmann, Holm and Puppe [29]. The $\mathbb{Z}_{2}$-actions are replaced by U(1)-actions, and $\mathbb{F}_{2}$-coefficient cohomology is replaced by $\mathbb{Q}$-coefficient cohomology. In the following set $\Gamma:=\mathrm{U}(1)$. Given a $\Gamma$-space $X$, denote by $X^{\Gamma}$ the set of $\Gamma$-fixed points and denote the $\Gamma$-equivariant cohomology of $X$ by

$$
H_{\Gamma}^{*}(X)=H^{*}\left(B_{\Gamma} X ; \mathbb{Q}\right), \quad \text { and } \quad H_{\Gamma}^{*}=H^{*}\left(\mathbb{C} P^{\infty} ; \mathbb{Q}\right)=\mathbb{Q}[u],
$$

where $u$ is the Euler class of the dual tautological bundle over $\mathbb{C} P^{\infty}$. We briefly recall the definition and main properties of circle spaces, and for further details we refer to [17] and [41].

Definition 4.1 A $\Gamma$-space $X$ is a circle space, if

- $X$ has nonzero cohomology in degrees $4 i$,

- there exists a degree-halving additive isomorphism $\kappa: H^{2 *}(X) \rightarrow H^{*}\left(X^{\Gamma}\right)$,

- there exists a Leray-Hirsch section (also known as cohomology extension of the fiber) $\sigma: H^{*}(X) \rightarrow H_{\Gamma}^{*}(X)$ which is multiplicative, and satisfies the restriction equation: for any $x \in H^{4 d}(X)$ :

$$
r(\sigma(x))=\kappa(x) u^{d}+\eta
$$

where $r: H_{\Gamma}^{*}(X) \rightarrow H_{\Gamma}^{*}\left(X^{\Gamma}\right) \cong H^{*}\left(X^{\Gamma}\right)[u]$, and $\eta$ is a $u$-polynomial of degree less than $d$.

Given a circle space $X$, it satisfies the following properties:

- $\kappa$ and $\sigma$ are multiplicative,

- a pair $(\kappa, \sigma)$ satisfying the restriction equation is unique, and they satisfy a naturality property with respect to equivariant maps between circle spaces.

Before stating the generalized Borel-Haefliger theorem we have to introduce a technical definition. By a good $\Gamma$-invariant cycle $Z \subseteq X$ we mean that 
- $Z$ is a $\Gamma$-invariant stratified submanifold which is a cycle,

- its top stratum $Z_{k}$ is $\Gamma$-invariant,

- the fixed point set $Z^{\Gamma}$ has a stratification with unique, connected top stratum $Z_{k}^{\Gamma}$ of some codimension $2 l$.

These technical conditions ensure that $Z^{\Gamma}$ is also a cycle, and that

$$
\left.[Z \subseteq X]_{\Gamma}\right|_{X^{\Gamma}}=w \cdot\left[Z^{\Gamma} \subseteq X^{\Gamma}\right]+\eta
$$

in $H_{\Gamma}^{*}\left(X^{\Gamma}\right) \cong H^{*}\left(X^{\Gamma}\right)[u]$, where $w=w_{0} \cdot u^{l}, w_{0} \in \mathbb{Z}$ is the weight of a representation and $\eta$ is a sum of $u$-monomials of degree less than $l$. This can be shown via the Excess Intersection Formula [45]. If $Z \subseteq X$ is a good $\Gamma$-invariant cycle satisfying codim $Z \subseteq X=2 \operatorname{codim} Z^{\Gamma} \subseteq X^{\Gamma}$, then we say in short that $Z$ is a halving cycle. To show that a space is a circle space, we can apply the following theorem [17, Theorem 4.1]:

Theorem 4.2 (Generalized Borel-Haefliger theorem) Let $\Gamma=\mathrm{U}(1)$ and let $X$ be a compact oriented manifold with a smooth $\Gamma$-action, whose rational cohomology groups have a basis of halving cycles $\left[Z_{i}\right] \in H^{4 k_{i}}(X)$. Assume that the $\Gamma$-equivariant normal bundle $\nu\left(X^{\Gamma} \hookrightarrow X\right)$ has only one weight $\lambda \in \mathbb{Z}$. Then $X$ is a circle space with $\kappa\left[Z_{i}\right]=\lambda^{k_{i}}\left[Z_{i}^{\Gamma}\right]$ and $\sigma\left[Z_{i}\right]=\left[Z_{i}\right]_{\Gamma}$.

In particular, the assignment sending $\left[Z_{i}\right]$ to $\left[Z_{i}^{\Gamma}\right]$ determines a degree-halving multiplicative isomorphism between $H^{2 *}(X ; \mathbb{Q})$ and $H^{*}\left(X^{\Gamma} ; \mathbb{Q}\right)$.

Remark 4.3 More generally, one can drop the assumption on orientability [17, Remark 3.24] and only assume that $X$ and $X^{\Gamma}$ satisfy $\mathbb{Q}$-Poincaré duality [1, Definition 5.1.1] and that their formal dimensions satisfy $\mathrm{fd}(X)=2 \mathrm{fd}\left(X^{\Gamma}\right)$. ( $X$ is a $\mathbb{Q}$-Poincaré duality space if $H^{\text {top }}(X ; \mathbb{Q}) \cong \mathbb{Q}$ and the pairing $H^{k}(X) \otimes H^{\text {top }-k}(X) \rightarrow H^{\text {top }}(X)$ is perfect; $\mathrm{fd}(X):=$ top degree.)

Our main examples of circle spaces are the real even flag manifolds $\mathrm{Fl}_{2 \mathcal{D}}^{\mathbb{R}}$. Introduce a $\Gamma$-action on real flag manifolds in $\mathbb{R}^{2 n}$ as follows: The identification of $\mathbb{R}^{2 n} \leftrightarrow \mathbb{C}^{n}$ as real $\Gamma$-representations induces an action on $\mathrm{Fl}_{\mathcal{E}}\left(\mathbb{R}^{2 n}\right), \mathcal{E}=\left(e_{1}, \ldots, e_{r}\right)$.

Theorem 4.4 Let $\Gamma:=\mathrm{U}(1)$. With the $\Gamma$-action introduced above, $\mathrm{Fl}_{2 \mathcal{D}}\left(\mathbb{R}^{2 n}\right)$ is a circle space, with $\Gamma$-fixed point set $\mathrm{Fl}_{\mathcal{D}}\left(\mathbb{C}^{n}\right)$. Furthermore

$$
\kappa\left[\sigma_{D I}^{\mathbb{R}}\right]=2^{|I|}\left[\sigma_{I}^{\mathbb{C}}\right]
$$

where $\left[\sigma_{I}^{\mathbb{C}}\right] \in H^{2|I|}\left(\mathrm{Fl}_{\mathcal{D}}\left(\mathbb{C}^{N}\right)\right)$.

Proof A representation theoretic computation involving the tangent bundles shows that the normal weights are all 2. By Theorem 4.2, it is enough to show that a) the Schubert cycles $\left[\sigma_{D I}\right]$ form a basis of rational cohomology, b) for an appropriate complete real flag $F_{\bullet}, Z=\sigma_{D I}^{\mathbb{R}}\left(F_{\bullet}\right)$ are good $U(1)$-invariant cycles satisfying codim $Z=$ $2 \operatorname{codim} Z^{\Gamma}$, and c) have $\mathrm{U}(1)$-fixed point set $Z^{\Gamma}=\sigma_{I}^{\mathbb{C}}\left(F_{\bullet}^{\mathbb{C}}\right)$. The $\left[\sigma_{D I}^{\mathbb{R}}\right]$ form a basis by Theorem 3.12, so a) holds. It remains to choose a flag $F_{\bullet}$ satisfying b) and c). 
b) Let $F_{\bullet}$ be a complete flag in $\mathbb{R}^{2 n}$, such that $F_{2 i}$ are $\Gamma$-invariant, and let $F_{\bullet}^{\mathbb{C}}$ denote the corresponding complex flag $\left(F_{0}, F_{2}, \ldots, F_{2 n}\right)$ in $\mathbb{C}^{n}$ by the identification $\mathbb{R}^{2 n} \leftrightarrow \mathbb{C}^{n}$. Then, $\sigma_{D I}^{\mathbb{R}}\left(F_{\bullet}\right)$ are halving cycles. Indeed, by the rank conditions (2.3), the complex points of $\sigma_{D I}^{\mathbb{R}}\left(F_{\bullet}\right)$ are the points of $\sigma_{I}^{\mathbb{C}}\left(F_{\bullet}^{\mathbb{C}}\right)$ since

$$
\operatorname{dim}_{\mathbb{R}}\left(W \cap W^{\prime}\right)=2 k \Longleftrightarrow \operatorname{dim}_{\mathbb{C}}\left(W \cap W^{\prime}\right)=k
$$

for any $\Gamma$-invariant subspaces $W, W^{\prime} \subseteq \mathbb{R}^{2 n}$. Therefore for this choice of $F_{\bullet}$, the $\sigma_{D I}^{\mathbb{R}}\left(F_{\bullet}\right)$ are $\Gamma$-invariant and $\left(\sigma_{D I}^{\mathbb{R}}\left(F_{\bullet}\right)\right)^{\bar{\Gamma}}=\sigma_{I}^{\mathbb{C}}\left(F_{\bullet}^{\mathbb{C}}\right)$, so c) holds. A dimension count shows

$$
\operatorname{codim}_{\mathbb{R}} \sigma_{D I}^{\mathbb{R}}\left(F_{\bullet}\right)=2 \operatorname{codim}_{\mathbb{R}} \sigma_{I}^{\mathbb{C}}\left(F_{\bullet}^{\mathbb{C}}\right)
$$

and since $\Omega_{I}^{\mathbb{C}}$ is the unique top stratum of $\sigma_{I}^{\mathbb{C}}$, the $\sigma_{D I}^{\mathbb{R}}$ are good U(1)-invariant cycles.

We obtain the following Corollary:

Corollary 4.5 (Littlewood-Richardson coefficients) In $H^{*}\left(\mathrm{Fl}_{2 \mathcal{D}} ; \mathbb{Q}\right)$ the structure constants are given by

$$
\left[\sigma_{D I}^{\mathbb{R}}\right] \cdot\left[\sigma_{D J}^{\mathbb{R}}\right]=\sum_{K} c_{I J}^{K}\left[\sigma_{D K}^{\mathbb{R}}\right]
$$

where $c_{I J}^{K}$ are the Littlewood-Richardson coefficients of the complex Schubert varieties:

$$
\left[\sigma_{I}^{\mathbb{C}}\right] \cdot\left[\sigma_{J}^{\mathbb{C}}\right]=\sum_{K} c_{I J}^{K}\left[\sigma_{K}^{\mathbb{C}}\right]
$$

Proof By the Theorem and multiplicativity of $\kappa$,

$$
\begin{aligned}
\kappa\left(\left[\sigma_{D I}^{\mathbb{R}}\right] \cdot\left[\sigma_{D J}^{\mathbb{R}}\right]\right) & =\kappa\left(\left[\sigma_{D I}^{\mathbb{R}}\right]\right) \cdot \kappa\left(\left[\sigma_{D J}^{\mathbb{R}}\right]\right)=2^{|I|+|J|}\left[\sigma_{I}^{\mathbb{C}}\right] \cdot\left[\sigma_{J}^{\mathbb{C}}\right] \\
& =2^{|I|+|J|} \sum_{K} c_{I J}^{K}\left[\sigma_{K}^{\mathbb{C}}\right] .
\end{aligned}
$$

Since $\kappa\left[\sigma_{D K}^{\mathbb{R}}\right]=2^{|K|}\left[\sigma_{K}^{\mathbb{C}}\right]$ and $|K|=|I|+|J|$, the Corollary follows.

The following corollaries follow analogously, using also the relation $\kappa p_{j}\left(S_{i}^{\mathbb{R}}\right)=$ $2^{j} c_{j}\left(S_{i}^{\mathbb{C}}\right)$, where $p_{j}, c_{j}$ denote Pontryagin and Chern classes respectively. For further details we refer to [41].

Corollary 4.6 (Giambelli formula type description) In $H^{*}\left(\mathrm{Fl}_{2 \mathcal{D}} ; \mathbb{Q}\right)$ the Schubert cycles can be expressed in terms of characteristic classes as follows:

$$
\left[\sigma_{D I}^{\mathbb{R}}\right]=q\left(p_{*}\left(S_{i}^{\mathbb{R}}\right)\right) \quad \Longleftrightarrow \quad\left[\sigma_{I}^{\mathbb{C}}\right]=q\left(c_{*}\left(S_{i}^{\mathbb{C}}\right)\right),
$$


that is the same polynomial $q \in \mathbb{Q}\left[x_{i}^{j}\right], i=1, \ldots, m, j=1, \ldots, d_{i}$ describes the double real Schubert classes and complex Schubert classes in terms of Pontryagin classes $p_{j}\left(S_{i}^{\mathbb{R}}\right)$ and Chern classes $c_{j}\left(S_{i}^{\mathbb{C}}\right)$.

\section{Corollary 4.7}

$$
\begin{aligned}
H^{*}\left(\mathrm{Fl}_{2 \mathcal{D}}^{\mathbb{R}}\right) & =\mathbb{Q}\left[p_{*}\left(S_{i}^{\mathbb{R}}\right)\right] / \mathcal{R}\left(p_{*}\left(S_{i}^{\mathbb{R}}\right)\right) \quad \Longleftrightarrow \quad H^{*}\left(\mathrm{Fl}_{\mathcal{D}}^{\mathbb{C}}\right) \\
& =\mathbb{Q}\left[c_{*}\left(S_{i}^{\mathbb{C}}\right)\right] / \mathcal{R}\left(c_{*}\left(S_{i}^{\mathbb{C}}\right)\right)
\end{aligned}
$$

where $\mathcal{R}\left(x_{*}^{i}\right)$ denotes an ideal in the variables $x_{j}^{i}$, that is the same polynomial relations hold in the two cohomology rings in terms of Pontryagin and Chern classes of the respective tautological bundles.

\section{Real Grassmannians}

The Casian-Kodama conjecture [11] concerned the cohomology ring structure of real Grassmannians and was stated as follows. For $(K, N)=(2 k, 2 n),(2 k+1,2 n+1)$ or $(2 k, 2 n+1)$ :

$$
H^{*}\left(\operatorname{Gr}_{K}\left(\mathbb{R}^{N}\right) ; \mathbb{Q}\right) \cong \mathbb{Q}\left[p_{1}, \ldots, p_{k}, p_{1}^{\prime}, \ldots, p_{n-k}^{\prime}\right] /\left(p_{*} \cdot p_{*}^{\prime}-1\right)
$$

where $p_{i}=p_{i}\left(S_{1}\right), p_{i}^{\prime}=p_{i}\left(Q_{1}\right), p_{*}=\sum_{i=0}^{k} p_{i}, p_{*}^{\prime}=\sum_{i=0}^{n-k} p_{i}^{\prime}$ and

$$
H^{*}\left(\operatorname{Gr}_{2 k+1}\left(\mathbb{R}^{2 n}\right) ; \mathbb{Q}\right) \cong \mathbb{Q}\left[p_{1}, \ldots, p_{k}, p_{1}^{\prime}, \ldots, p_{n-k}^{\prime}, r\right] /\left(p_{*} \cdot p_{*}^{\prime}-1, r^{2}\right)
$$

where $r$ is the Schubert class corresponding to the $L$-shape Young diagram: $(n-$ $k, 1^{k-1}$ ). The conjecture of Casian and Kodama (5.1) and (5.2) on the cohomology ring was completely settled even equivariantly using different methods by Takeuchi, He, Sadykov and Carlson [10,30,47,50], see Q3) in Sect. 1.4.

Another kind of description of the ring structure is obtained by giving an additive basis in Schubert cycles and by determining their structure constants. These results are due to Pontryagin, Fuchs, Finashin and Kharlamov [23,44] and [20]. We briefly summarize how to obtain these results from the previous computations.

\subsection{Additive structure}

A convenient way to parametrize the Schubert varieties in Grassmannians is by Young diagrams $\lambda \subseteq K \times(N-K)$. One has the following conversion formulas between $\lambda \subseteq K \times(N-K)$ and $I \in\left(\begin{array}{l}N \\ K\end{array}\right)$ :

$$
\lambda_{j}=N-K+j-I_{j}, \quad I_{j}=N-K+j-\lambda_{j} .
$$


Fig. 4 The $L$-operation on $\lambda=(3,1,0) \subseteq 3 \times 4$

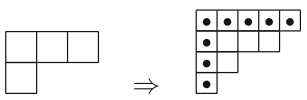

for $j=1, \ldots, K$. Before stating the Schubert cycle description of $H^{*}\left(\mathrm{Gr}_{K}\left(\mathbb{R}^{N}\right) ; \mathbb{Q}\right)$ it is convenient to introduce the $L$-operation on Young diagrams: given $\lambda \subseteq K \times(N-K)$, let $L \lambda \subseteq(K+1) \times(N-K+1)$ be the partition

$$
L \lambda:=\left(N-K+1, \lambda_{1}+1, \lambda_{2}+1, \ldots, \lambda_{K}+1\right) .
$$

In terms of Young diagrams, the diagram contains the first row and column, and the complement of this L-shape is the Young diagram $\lambda$, see Fig. 4 (the added L-shape is marked with bullet points). We call the corresponding Schubert varieties $\sigma_{L \lambda} L$ Schubert varieties. Recall that $D \lambda \subseteq 2 k \times 2(n-k)$ denotes the double of a Young diagram $\lambda \subseteq k \times(n-k)$ defined earlier (Fig. 2).

\section{Theorem 5.1}

$H^{*}\left(\operatorname{Gr}_{K}\left(\mathbb{R}^{N}\right) ; \mathbb{Q}\right)= \begin{cases}\mathbb{Q}\left\langle\left[\sigma_{D \lambda}\right],\left[\sigma_{L(D \lambda)}\right]: \lambda \subseteq k \times(n-k)\right\rangle & \text { if } N \text { is even and } K \text { is odd } \\ \mathbb{Q}\left\langle\left[\sigma_{D \lambda}\right]: \lambda \subseteq k \times(n-k)\right\rangle & \text { else. }\end{cases}$

where $k=\lfloor K / 2\rfloor, n=\lfloor N / 2\rfloor$.

Proof In terms of Young diagrams, the incidence coefficients (3.6) are the following (see also [11]). If $\mu$ is a partition obtained from $\lambda$ by increasing $\lambda_{j}$ by 1 , then

$$
\left[\sigma_{\lambda}, \sigma_{\mu}\right]= \begin{cases}0 & \lambda_{j}-j \text { odd } \\ \pm 2 & \lambda_{j}-j \text { even }\end{cases}
$$

Then $\sigma_{\lambda}$ is a cycle if and only if for all partitions $\mu$ obtained by increasing $\lambda_{j}$ by $1, \lambda_{j}-j$ is odd. This implies that double Schubert varieties $\sigma_{D \lambda}$ and double LSchubert varieties $\sigma_{L(D \lambda)}$ are cycles - possibly torsion. However, $\left[\sigma_{D \lambda}, \sigma_{\mu}\right]=0$ for all $\operatorname{dim} \sigma_{\mu}=\operatorname{dim} \sigma_{D \lambda}+1$, so the $\left[\sigma_{D \lambda}\right]$ do not appear in any relations and therefore are linearly independent. A similar computation shows that if $K$ odd and $N$ even, $\left[\sigma_{L(D \lambda)}\right]$ appear with zero coefficient in every incidence relation, so these classes are linearly independent. By counting ranks, (5.1) and (5.2) imply that

$$
\operatorname{dim}_{\mathbb{Q}} H^{*}\left(\operatorname{Gr}_{K}\left(\mathbb{R}^{N}\right) ; \mathbb{Q}\right)= \begin{cases}2\left(\begin{array}{l}
n \\
k
\end{array}\right) & N \text { even } K \text { odd } \\
\left(\begin{array}{l}
n \\
k
\end{array}\right) & \text { else. }\end{cases}
$$

which implies that these Schubert classes form a basis.

\subsection{Multiplicative structure}

The $\operatorname{Gr}_{K}\left(\mathbb{R}^{N}\right)$ are circle spaces unless $K$ is odd and $N$ is even. If $K$ and $N$ is even, this is a special case of Theorem 4.4. In particular, the Giambelli and Pieri formulas 
hold by replacing all $\left[\sigma_{\lambda}^{\mathbb{C}}\right]$ with $\left[\sigma_{D \lambda}^{\mathbb{R}}\right]$ in the formulas. The remaining cases: $K$ odd $N$ even and $K$ even $N$ odd are both nonorientable. We now proceed to show that they are also circle spaces.

Proposition 5.2 The natural inclusions induce isomorphisms of cohomology with rational coefficients

$$
H^{*}\left(\operatorname{Gr}_{2 k}\left(\mathbb{R}^{2 n}\right)\right) \cong H^{*}\left(\operatorname{Gr}_{2 k}\left(\mathbb{R}^{2 n+1}\right)\right) \cong H^{*}\left(\operatorname{Gr}_{2 k+1}\left(\mathbb{R}^{2 n+1}\right)\right)
$$

and the descriptions given in Corollaries 4.5, 4.6, 4.7 hold for $\operatorname{Gr}_{2 k}\left(\mathbb{R}^{2 n+1}\right)$ and $\mathrm{Gr}_{2 k+1}\left(\mathbb{R}^{2 n+1}\right)$ as well.

Proof Let $F_{\bullet}^{\mathbb{R}} \in \mathrm{Fl}\left(\mathbb{R}^{2 n+1}\right)$ be the standard complete flag. Identify $\mathbb{R}^{2 n+1}=\mathbb{R} \oplus \mathbb{C}^{n}$ as $\Gamma=\mathrm{U}(1)$-representations; let the trivial representation be the first coordinate $\mathbb{R}=\left\langle e_{1}\right\rangle$. This induces actions on $\mathrm{Gr}_{2 k+1}\left(\mathbb{R}^{2 n+1}\right)$ and $\mathrm{Gr}_{2 k}\left(\mathbb{R}^{2 n+1}\right)$, whose fixed point set can be identified with $\mathrm{Gr}_{k}\left(\mathbb{C}^{n}\right)$. By the definition of the U(1)-action, $F_{\bullet}^{\mathbb{C}}=\left(\left\langle e_{2}, e_{3}\right\rangle,\left\langle e_{2}, e_{3}, e_{4}, e_{5}\right\rangle, \ldots,\left\langle e_{2}, \ldots, e_{2 n+1}\right\rangle\right)$ is a complete complex flag $F_{\bullet}^{\mathbb{C}} \in \mathrm{Fl}\left(\mathbb{C}^{n}\right)$. The rank description (2.3) implies that double Schubert varieties $\sigma_{D \lambda}\left(F_{\bullet}^{\mathbb{R}}\right)$ are halving cycles, with fixed point set $\sigma_{\lambda}^{\mathbb{C}}\left(F_{\bullet}^{\mathbb{C}}\right)$.

We will apply Theorem 4.2 , but since $\mathrm{Gr}_{K}\left(\mathbb{R}^{2 n+1}\right)$ are not orientable, we have to verify the conditions described in Remark 4.3. By He's Theorem A.3, any flag manifold is a Poincaré duality space, and $\operatorname{fd}(X)=4 k(n-k)$ and $\operatorname{fd}\left(X^{\Gamma}\right)=2 k(n-k)$. So the conditions of Remark 4.3 are satisfied and one can apply Theorem 4.2 to conclude.

Finally, the natural inclusions induce the isomorphisms. Indeed, the characteristic classes are mapped into each other via the natural inclusions, so there is a system of generators mapped into a system of generators with the same relations. For example,

$$
i: \mathrm{Gr}_{2 k}\left(\mathbb{R}^{2 n}\right) \hookrightarrow \mathrm{Gr}_{2 k}\left(\mathbb{R}^{2 n+1}\right)
$$

pulls back $i^{*} p_{j}\left(S^{2 n+1}\right)=p_{j}\left(S^{2 n}\right)$ and $i^{*} p_{j}\left(Q^{2 n+1}\right)=p_{j}\left(Q^{2 n}\right)$ (even though the pullback $i^{*} Q^{2 n+1}=Q^{2 n} \oplus \varepsilon$.)

Proposition 5.3 The structure constants of $\left[\sigma_{D \lambda}\right]$ and $\left[\sigma_{L(D \lambda)}\right]$ in $H^{*}\left(\mathrm{Gr}_{2 k+1}\left(\mathbb{R}^{2 n}\right)\right)$ are completely determined by the Littlewood-Richardson structure constants of $\left[\sigma_{D \lambda}\right]$ (Corollary 4.5) and

$$
\left[\sigma_{D \lambda}\right] \cdot\left[\sigma_{L 0}\right]=\left[\sigma_{L(D \lambda)}\right], \quad\left[\sigma_{L 0}\right]^{2}=0
$$

Proof Since $\left[\sigma_{L 0}\right] \in H^{2 n-1}$ lives in odd degree, and multiplication is graded commutative, $\left[\sigma_{L 0}\right]^{2}$ is 2-torsion, therefore zero rationally.

To show $\left[\sigma_{D \lambda}\right] \cdot\left[\sigma_{L 0}\right]=\left[\sigma_{L(D \lambda)}\right]$, we use the following lemma.

Lemma 5.4 In $\mathrm{Gr}_{k}\left(\mathbb{R}^{n}\right)$, there exist transverse flags $E_{\bullet}, F_{\bullet}$ and a flag $G_{\bullet}$, such that

$$
\sigma_{L 0}\left(E_{\bullet}\right) \cap \sigma_{\lambda}\left(F_{\bullet}\right)=\sigma_{L \lambda}\left(G_{\bullet}\right)
$$


Proof Let $E_{\bullet}$ be the standard flag, $F_{\bullet}$ the opposite flag

$$
F_{\bullet}=\left\langle e_{n}\right\rangle \subseteq\left\langle e_{n}, e_{n-1}\right\rangle \subseteq \ldots \subseteq\left\langle e_{n}, e_{n-1}, \ldots e_{2}\right\rangle \subseteq\left\langle e_{n}, e_{n-1}, \ldots, e_{1}\right\rangle
$$

and $G_{\bullet}$ be obtained from $F_{\bullet}$ by exchanging $e_{1}$ and $e_{n}$ :

$$
G_{\bullet}=\left\langle e_{1}\right\rangle \subseteq\left\langle e_{1}, e_{n-1}\right\rangle \subseteq \ldots \subseteq\left\langle e_{1}, e_{n-1}, \ldots e_{2}\right\rangle \subseteq\left\langle e_{1}, e_{n-1}, \ldots, e_{3}, e_{2}, e_{n}\right\rangle
$$

$E_{\bullet}$ and $F_{\bullet}$ are transverse flags, which imply that the Schubert varieties $\sigma_{L 0}\left(E_{\bullet}\right) \cap$ $\sigma_{\lambda}\left(F_{\bullet}\right)$ intersect transversely. The rank conditions defining $\sigma_{L 0}\left(E_{\bullet}\right)$ translate to

$$
U \in \sigma_{L 0} \quad \Longleftrightarrow \quad E_{1} \subseteq U \subseteq E_{n-1}
$$

in particular, $\sigma_{L 0}\left(E_{\bullet}\right)$ is a subGrassmannian $\operatorname{Gr}_{k}\left(\mathbb{R}^{n-2}\right)$. The following observation allows us to conclude: if $E_{1} \subseteq U \subseteq E_{n-1}$, then

$$
\operatorname{dim}\left(U \cap F_{j}\right)=k \quad \Longleftrightarrow \quad \operatorname{dim}\left(U \cap G_{j}\right)=k+1
$$

By comparing the rank conditions defining $\sigma_{\lambda}$ and $\sigma_{L \lambda}$ the two sides of (5.5) are equal.

Remark 5.5 (i) The previous lemma was stated for arbitrary $k$ and $n$; a simple verification shows that the subGrassmannian $\sigma_{L 0}$ is always coorientable and therefore a cycle. However, the computation which shows that it appears in every incidence relation with zero coefficient only holds if $k$ is odd and $n$ is even, otherwise [ $\left.\sigma_{L 0}\right]$ is a 2-torsion element.

(ii) If one defines the usual U(1)-action on $\mathbb{R}^{2 n}$ by identifying it with $\mathbb{C}^{n}$, the induced action on $\operatorname{Gr}_{2 k+1}\left(\mathbb{R}^{2 n}\right)$ has no fixed points; indeed, $\mathbb{C}^{n}$ has no real odd dimensional invariant subspaces. $\operatorname{Gr}_{2 k+1}\left(\mathbb{R}^{2 n}\right)$ has zero Euler characteristic so it cannot be a circle space.

\section{Integer coefficients and Steenrod squares}

It is a classical result that all torsion in $H^{*}\left(\mathrm{Gr}_{k}\left(\mathbb{R}^{\infty}\right) ; \mathbb{Z}\right)$ is of order 2 , see e.g. [6, Theorem 24.7]. By a theorem of Ehresmann [16, p. 81], this also holds in the case of finite Grassmannians. Therefore the rational and mod 2 reductions of an integer cohomology class completely determine its value. In particular, one can express the integer classes of Schubert cycles in terms of Pontryagin classes and Bockstein's of Stiefel-Whitney classes, see Theorem 6.7 below. In this chapter, we extend Ehresmann's theorem to the case of even real flag manifolds $\mathrm{Fl}_{2} \mathcal{D}$, and conjecture that it holds in general:

Theorem 6.1 For even real flag manifolds $2 \operatorname{Tor}\left(H^{*}\left(\mathrm{Fl}_{2 \mathcal{D}} ; \mathbb{Z}\right)\right)=0$, i.e. every torsion element of $H^{*}\left(\mathrm{Fl}_{2 \mathcal{D}} ; \mathbb{Z}\right)$ is of order 2. In particular, if $\beta$ denotes the Bockstein 
homomorphism and $H_{\text {free }}^{*}$ denotes the free part:

$$
H^{*}\left(\mathrm{Fl}_{2 \mathcal{D}} ; \mathbb{Z}\right)=H_{\text {free }}^{*}\left(\mathrm{Fl}_{2 \mathcal{D}} ; \mathbb{Z}\right) \bigoplus \operatorname{Im} \beta
$$

After some preparation in Sect. 6.3, we prove this Theorem in Sect. 6.3.3 using Proposition 6.9 of Borel and Hirzerbruch which requires computing the Poincaré polynomial of Bockstein cohomology $P_{\beta}$.

For a general space $X$, one way to compute $H^{*}(X ; \mathbb{Z})$ is by computing the mod $p$ Bockstein spectral sequences. Degeneration of the mod 2 Bockstein spectral sequence on the $E_{2}$-page is equivalent to saying that the 2-primary part of $H^{*}(X ; \mathbb{Z})$ consists entirely of elements of order 2 . The differential $d_{1}$ on the first page $E_{1}^{p, q}=H^{p+q}\left(X ; \mathbb{F}_{2}\right)$ of the mod 2 Bockstein spectral sequence is the first Steenrod square $\mathrm{Sq}^{1}$. In order to compute the $E_{2}$-page, we will give a combinatorial description of the $\mathrm{Sq}^{1}$-action on the additive basis of Schubert cycles, which is strongly related to the incidence coefficients computed earlier, see Proposition 6.3. We will then use this description to compute the $E_{2}$-page of the Bockstein spectral sequence.

The cohomology groups $H^{*}\left(\mathrm{Fl}_{2 \mathcal{D}} ; \mathbb{Z}\right)$ then can be completely determined by using this theorem, the Cartan description of Section A and the universal coefficient theorem. This is a combinatorial computation which can be carried out similarly as was done in [31] for the case of Grassmannians. It relies on the following observation: if all torsion in $H^{*}(X ; \mathbb{Z})$ is of order two, then $P_{\text {Tor }}=\frac{t}{t+1}\left(P_{2}-P_{0}\right)$, where $P_{\text {Tor }}$ denotes the Poincaré polynomial of the ranks of $\mathbb{Z}_{2}$ 's in $H^{*}(X ; \mathbb{Z}), P_{2}$ and $P_{0}$ denote the mod 2 and rational coefficient Poincaré polynomials of $X$.

Proposition 6.2 Let $\mathcal{D}=\left(d_{1}, \ldots, d_{r}\right), N=\sum d_{i}$. The Poincaré polynomial of the ranks of $\mathbb{Z}_{2}$ 's in $H^{*}\left(\mathrm{Fl}_{2 D}^{\mathbb{R}} ; \mathbb{Z}\right)$ is given by

$$
P_{\mathrm{Tor}}\left(\mathrm{Fl}_{2 \mathcal{D}}\right)=\frac{t}{t+1}\left(\frac{\prod_{j=1}^{2 N}\left(1-t^{j}\right)}{\prod_{i=1}^{r} \prod_{j=1}^{2 d_{i}}\left(1-t^{j}\right)}-\frac{\prod_{j=1}^{N}\left(1-t^{4 j}\right)}{\prod_{i=1}^{r} \prod_{j=1}^{d_{i}}\left(1-t^{4 j}\right)}\right) .
$$

Proof The Poincaré polynomial $P_{2}$ of mod 2 cohomology is a classical result of Borel [5] and rational coefficient Poincaré polynomial $P_{0}$ follows from the He's Theorem A.3, [32].

\subsection{Steenrod squares}

Lenart [40] computed the Steenrod coefficients $c_{\lambda \mu}^{k} \in \mathbb{F}_{2}$ in complex Grassmannians:

$$
\mathrm{Sq}^{k}\left[\sigma_{\lambda}\right]=\sum_{\mu} c_{\lambda \mu}^{k}\left[\sigma_{\mu}\right]
$$

As he remarks, the Steenrod coefficients are obstructions for the triviality of the attaching maps: if $c_{\lambda \mu}^{k}$ is nonzero, then the corresponding attaching map is nontrivial. He also gives an example [40, Section 6], where the converse does not hold. The more 
general case of Steenrod coefficients $c_{I J}^{k}$ for arbitrary flag manifolds of simply connected, semisimple Lie groups (and arbitrary $p$ ) was determined by Duan and Zhao [14]. Notice that the distinction of real and complex is irrelevant; by results of [22] and [54]:

$$
\mathrm{Sq}^{2 k}\left[\sigma_{I}^{\mathbb{C}}\right]=\sum_{J} c_{I J}^{k}\left[\sigma_{J}^{\mathbb{C}}\right] \quad \Longleftrightarrow \quad \mathrm{Sq}^{k}\left[\sigma_{I}^{\mathbb{R}}\right]=\sum_{J} c_{I J}^{k}\left[\sigma_{J}^{\mathbb{R}}\right]
$$

On the other hand, for $k=1$, the Steenrod coefficients $c_{I J}^{1}$ for real flag manifolds vanish if and only if the incidence coefficients $\left[\Omega_{I}, \Omega_{J}\right]$ vanish:

Proposition 6.3 For all $I, J \in \operatorname{OSP}(\mathcal{D})$, set $c_{I J}:=\left|\left[\Omega_{I}, \Omega_{J}\right] / 2\right| \in \mathbb{F}_{2}$ where $\left[\Omega_{I}, \Omega_{J}\right]$ are the incidence coefficients, described in (3.6). Then

$$
\mathrm{Sq}^{1}\left[\sigma_{I}\right]=\sum c_{I J}\left[\sigma_{J}\right]
$$

For a related statement for the case of Grassmannians in the algebraic geometric context (Chow-Witt ring) see [57, Theorem 1.1]. The Proposition follows from the following classical viewpoint on Bockstein homomorphisms (see e.g. [42]):

Lemma 6.4 If $(C, d)$ is a cochain complex, then the Bockstein homomorphism of $(C, 2 d)$

$$
\beta: H^{*}\left(C \otimes \mathbb{F}_{2}, 2 d \otimes \mathbb{F}_{2}=0\right) \rightarrow H^{*+1}(C, 2 d)
$$

satisfies

$$
\beta[\rho z]=[d z] \in H^{*+1}(C, 2 d),
$$

for all $z \in C$, where $\rho: C \rightarrow C \otimes \mathbb{F}_{2}$ is the mod 2 reduction.

The proof is an unraveling of the definition of the Bockstein homomorphism.

Proof (Proof of Proposition) Apply the Lemma to the Vassiliev complex (all coefficients are \pm 2 ) and use the classical fact that the mod 2 reduction of the Bockstein homomorphism is $\mathrm{Sq}^{1}$.

Thus the knowledge of the incidence coefficients (3.6) yields a combinatorial formula for the Steenrod coefficients of $\mathrm{Sq}^{1}$ in an arbitrary partial flag manifold in terms of Schubert cycles.

Remark 6.5 There is a geometric proof of Proposition 6.3. This involves the Thom-Wu theorem which states that if $i: Z \hookrightarrow X$ is a smooth submanifold, then $\mathrm{Sq}^{k}[Z]=$ $i_{!} w_{k}\left(v_{i}\right)$. Together with the description of $\left[\Omega_{I}, \Omega_{J}\right]$ in Proposition 3.1, this proves Proposition 6.3. 


\subsection{Integral cycles}

Before proving Theorem 6.1, we deduce some easy consequences about integral cycles.

Proposition 6.6 Let $X$ be a manifold satisfying $2 \operatorname{Tor}\left(H^{*}(X ; \mathbb{Z})\right)=0$. Then every integral cycle $[Z] \in H^{*}(X ; \mathbb{Z})$ is one of the two following types

- $[Z] \in \operatorname{Im} \beta$, in which case $2[Z]=0$ or

- $[Z \bmod 2] \neq 0 \in \operatorname{ker~Sq}^{1} / \mathrm{Im} \mathrm{Sq}^{1}$, in which case $[Z] \neq 0$ rationally.

In particular, by Lemma 6.4, the 2-torsion part of $H^{*}\left(\mathrm{Fl}_{2 \mathcal{D}} ; \mathbb{Z}\right)$ is generated by cycles of the form $\left[d \Omega_{I} / 2\right]$.

We relate Schubert cycles to integral characteristic classes. Denote a StiefelWhitney monomial in $H^{*}\left(\mathrm{Fl}_{\mathcal{D}}\left(\mathbb{R}^{N}\right) ; \mathbb{F}_{2}\right)$ by

$$
w_{M}=\prod_{i, j} w_{i}\left(D_{j}\right)^{m_{i j}},
$$

where $M=\left(m_{i j}\right) \in \mathbb{N}^{N}$ denotes a multiindex $\left(N=\sum_{i=1}^{r} d_{i}\right)$. Then every element of $H^{*}\left(\mathrm{Fl}_{\mathcal{D}}\left(\mathbb{R}^{N}\right) ; \mathbb{F}_{2}\right)$ can be written as a sum of $w_{M}$ 's (not necessarily uniquely!), write $\sum w_{M}$ for a generic element. Then $\sum w_{M}$ is the mod 2 reduction of a 2-torsion class in $H^{*}\left(\mathrm{Fl}_{\mathcal{D}} ; \mathbb{Z}\right)$ iff $\sum w_{M} \in \mathrm{Im} \mathrm{Sq}^{1}$. For such elements, denote by $\sum v_{M}$ the unique second order element in $\operatorname{Im} \beta \subseteq H^{*}\left(\mathrm{Fl}_{2 \mathcal{D}} ; \mathbb{Z}\right)$ whose mod 2 reduction equals $\sum w_{M}$.

A consequence of Theorem 6.1 is that from the $\bmod 2$ and rational descriptions of double Schubert classes $\left[\sigma_{D I}\right]$, one can express the integer classes $\left[\sigma_{D I}\right]$ in terms of characteristic classes. Using the previous notations, we can give a formula:

Theorem 6.7 The integral classes $\left[\sigma_{D I}\right] \in H^{*}\left(\mathrm{Fl}_{2 \mathcal{D}}\left(\mathbb{R}^{N}\right) ; \mathbb{Z}\right)$ equal

$$
\left[\sigma_{D I}\right]=s_{I}\left(p_{i}\left(D_{j}\right)\right)+\left\{s_{D I}\left(v_{i, j}\right)-s_{I}\left(v_{i, 2 j}^{2}\right)\right\}
$$

$j=1, \ldots, r$ and $i=1, \ldots, d_{j}$ where $s_{I}\left(x_{i, j}\right)$ denotes the Schubert polynomial in the variables $x_{i, j}$ (in terms of elementary symmetric polynomials [3, Sect. 5]).

Here $s_{D I}\left(w_{i}\left(D_{j}\right)\right)-s_{I}\left(w_{2 i}\left(D_{j}\right)^{2}\right) \in H^{*}\left(\mathrm{Fl}_{2 \mathcal{D}} ; \mathbb{F}_{2}\right)$ is in $\mathrm{Im} \mathrm{Sq}^{1}$, and therefore lifts to a unique second order element denoted $\left\{s_{D I}\left(v_{i, j}\right)-s_{I}\left(v_{i, 2 j}^{2}\right)\right\} \in H^{*}\left(\mathrm{Fl}_{2 \mathcal{D}} ; \mathbb{Z}\right)$.

Proof The rational class is $\left[\sigma_{D I}\right]=s_{I}\left(p_{i, j}\right)$ by the generalized Borel-Haefliger theorem (Corollary 4.6) and the mod 2 class is $s_{D I}\left(w_{i, j}\right)$ by the classical mod 2 BorelHaefliger theorem. The theorem then follows from the fact that the mod 2 reduction of the $j$ th integral Pontryagin class $p_{j}$ is $w_{2 j}^{2}$.

Remark 6.8 Contrary to what the notation might suggest, $\left\{s_{D I}\left(v_{i, j}\right)-s_{I}\left(v_{i, 2 j}^{2}\right)\right\}$ is not a polynomial in some classes $v_{i, j}$; it is an element, whose mod 2 reduction is the polynomial $s_{D I}\left(w_{i, j}\right)-s_{I}\left(w_{i, 2 j}^{2}\right)$. Determining the class $\left[\sigma_{D I}\right]$ in terms of multiplicative generators of $H^{*}\left(\mathrm{Fl}_{2 \mathcal{D}} ; \mathbb{Z}\right)$ is a challenge we will not consider. 


\subsection{Proof of Theorem 6.1}

In the rest of this section we prove Theorem 6.1, but first we recall some generalities about the Bockstein spectral sequence and the Bockstein cohomology of a space.

\subsubsection{Bockstein cohomology}

The Bockstein cohomology of a topological space $X$ can be defined as follows: since $\mathrm{Sq}^{1}: H^{*}\left(X ; \mathbb{F}_{2}\right) \rightarrow H^{*+1}\left(X ; \mathbb{F}_{2}\right)$ satisfies $\mathrm{Sq}^{1} \circ \mathrm{Sq}^{1}=0$, one can regard $H^{*}\left(X ; \mathbb{F}_{2}\right)$ as a chain complex and compute its cohomology

$$
H_{\beta}^{*}(X):=H^{*}\left(H^{*}\left(X ; \mathbb{F}_{2}\right) ; \mathrm{Sq}^{1}\right)
$$

Since $\mathrm{Sq}^{1}$ is the differential $d_{1}$ on the page $E_{1}^{p, q}=H^{p+q}\left(X ; \mathbb{F}_{2}\right)$ of the $\bmod 2$ Bockstein spectral sequence, so $H_{\beta}^{*}(X)$ is just the $E_{2}$-page of the Bockstein spectral sequence. Denote by $P_{\beta}(X)$ the Poincare polynomial of $H_{\beta}^{*}(X)$ and by $P_{0}(X)$ the Poincaré polynomial of $H^{*}(X ; \mathbb{Q})$. We will use the following Proposition of BorelHirzebruch, [4] Lemma 30.4 and 30.5 (1), cf. also [6]:

Proposition 6.9 If $H^{*}(X ; \mathbb{Z})$ is finitely generated, then the 2-primary component of $H^{*}(X ; \mathbb{Z})$ consists of elements of order 2 (i.e. the Bockstein spectral sequence degenerates) if and only if

$$
P_{\beta}(X)=P_{0}(X)
$$

Borel and Hirzebruch show in $[4,30.5]$ that in the case of infinite Grassmannians $G_{N}=\operatorname{Gr}_{N}\left(\mathbb{R}^{\infty}\right)$

$$
P_{0}\left(G_{N}\right)=P_{\beta}\left(G_{N}\right)=\prod_{i=1}^{\left\lfloor\frac{N}{2}\right\rfloor} \frac{1}{1-t^{4 i}} .
$$

In order to compute $P_{\beta}, \mathbb{F}_{2}\left[w_{1}, \ldots, w_{N}\right]$ is decomposed into the tensor product of subalgebras $A_{i}$ each invariant under $\mathrm{Sq}^{1}$. The Poincare polynomial $P_{\beta}$ is then the product of the Poincaré polynomials of $A_{i}: P_{\beta}=\prod_{i} P_{A_{i}}$. The generators of $H_{\beta}^{*}$ are also identified to be $\left[w_{2 i}^{2}\right]$.

We will reduce the finite case to the infinite case, so let us discuss the infinite version of flag manifolds. Given $\mathcal{D}=\left(d_{1}, \ldots, d_{r}\right)$, let

$$
\mathcal{D}^{m}=\left(d_{1}, \ldots, d_{r-1}, d_{r}+m\right)
$$

$m$ possibly infinite. Note that $\mathrm{Fl}_{\mathcal{D}^{\infty}}$ is the classifying space of the parabolic subgroup $P=\operatorname{GL}\left(d_{1}, \ldots, d_{r-1}\right)$ (using the notation of Sect. 2.2). 


\section{Proposition 6.10}

$$
P_{\beta}\left(\mathrm{Fl}_{2 \mathcal{D}^{\infty}}\right)=\prod_{i=1}^{r-1} \prod_{j=1}^{d_{i}} \frac{1}{1-t^{4 j}}=P_{0}\left(\mathrm{Fl}_{2 \mathcal{D}^{\infty}}\right)
$$

and $H_{\beta}^{*}\left(\mathrm{Fl}_{2 \mathcal{D}^{\infty}}\right)$ is generated by $\left[w_{2 j}^{2}\left(D_{i}\right)\right]$. In particular, all torsion of $H^{*}\left(\mathrm{Fl}_{2 \mathcal{D}} ; \mathbb{Z}\right)$ is of order 2 .

Proof Using that

$$
H^{*}\left(\mathrm{Fl}_{2 \mathcal{D}}{ }^{\infty}\right)=\mathbb{F}_{2}\left[w_{j}\left(D_{i}\right): i=1, \ldots, r-1, j=1, \ldots, d_{i}\right]
$$

this follows from (6.1), similarly to Borel's computations [6, pp. 85-86].

In the finite case, matters are complicated by the relations, since such a decomposition into subalgebras does not exist. This is why we will use the additive (Schubert basis) description of the cohomology groups, together with the combinatorial description of $\mathrm{Sq}^{1}$ (Proposition 6.3).

\subsubsection{Stabilization of Schubert classes}

Next we discuss stabilization of Schubert classes. With the notation $\mathcal{D}^{m}$ introduced in (6.2), we have direct sum maps (see Sect. 2.2.3):

$$
\mathcal{K}_{m}: \mathrm{Fl}_{\mathcal{D}}\left(\mathbb{R}^{N}\right) \hookrightarrow \mathrm{Fl}_{\mathcal{D}^{m}}\left(\mathbb{R}^{N+m}\right)
$$

The current indexing of the Schubert cycles does not satisfy nice stability properties, so from now on we will change the convention of indexing the Schubert varieties $\sigma_{I}$, $I \in \operatorname{OSP}(\mathcal{D})$. Let $\vee^{\vee}: \operatorname{OSP}(\mathcal{D}) \rightarrow \operatorname{OSP}(\mathcal{D})$ be the involution defined as follows: for $I \in \operatorname{OSP}(\mathcal{D})$, replace each element $k \in I_{j}$ by $N+1-k$ :

$$
I_{j}^{\vee}:=\left\{N+1-k: k \in I_{j}\right\}
$$

for all $j$, where $|\mathcal{D}|=N$. Let $\sigma_{I}:=\sigma_{I^{\vee}}$ and $\boldsymbol{\Omega}_{I}:=\Omega_{I^{\vee}}$ for all $I \in \operatorname{OSP}(\mathcal{D})$. For complete flag manifolds $\mathcal{D}=\left(1^{N}\right)$, this indexing convention agrees with [24], see also [26, p. 20]. With the new conventions $\ell(I)=\operatorname{codim} \sigma_{I}$ (for the notation $\ell(I)$ see (2.4)) and $\boldsymbol{\Omega}_{I}$ and $\boldsymbol{\Omega}_{J}$ are adjacent if $J$ is obtained from $I$ by exchanging $a \in I_{\alpha}$ with some $b \in I_{\beta}, a<b, \alpha<\beta$. The incidence relations are (for the notation $T_{I}(a, b)$ see (3.5)):

$$
\left[\boldsymbol{\Omega}_{I}, \boldsymbol{\Omega}_{J}\right]= \begin{cases}0, & T_{I}(a, b) \text { even } \\ \pm 2, & T_{I}(a, b) \text { odd }\end{cases}
$$


With this indexing convention, Schubert classes mod 2 satisfy the following stabilization property (this can be shown by a transversality argument):

$$
\mathcal{K}_{m}^{*}\left[\sigma_{J}\right]= \begin{cases}{\left[\sigma_{I}\right]} & \text { if } J=I^{m} \\ 0 & \text { else, }\end{cases}
$$

where for $I \in \operatorname{OSP}(\mathcal{D})$, define

$$
I^{m}:=\left(I_{1}, I_{2}, \ldots, I_{r-1}, I_{r} \cup\{N+1, \ldots, N+m\}\right) .
$$

In particular,

$$
\operatorname{ker} \mathcal{K}_{m}^{*}=\left\langle\left[\sigma_{I}\right]: \exists k>N: k \notin I_{r}\right\rangle
$$

Remark 6.11 For $I \in \operatorname{OSP}(\mathcal{D})$, stabilization implies that there exists $s_{I} \in$ $H^{*}\left(\mathrm{Fl}_{\mathcal{D}^{\infty}} ; \mathbb{F}_{2}\right)$, such that $K_{\infty}^{*} s_{I}=\left[\sigma_{I}\right]$ and factors through $\left[\sigma_{I^{m}}\right]$ for all $m$. Since $\mathrm{Fl}_{\mathcal{D}^{\infty}}$ is the classifying space of $\operatorname{GL}\left(d_{1}, \ldots, d_{r-1}\right), s_{I} \in H^{*}\left(\mathrm{Fl}_{\mathcal{D}^{\infty}}\right)$ is a polynomial $q\left(w_{i}^{j}\right)$ in the universal Stiefel-Whitney classes $w_{i}^{j}$, and since $\mathcal{K}_{\infty}^{*} w_{i}^{j}=w_{i}\left(D_{j}\right)$, stabilization provides universal formulas $\left[\sigma_{I^{m}}\right]=q\left(w_{i}\left(D_{j}\right)\right)$ in terms of characteristic classes (the minimal Schubert polynomials, see [3, Sect. 5]).

Stabilization also implies that there exists a universal formula for Steenrod squares in terms of Schubert cycles: there exist $c_{I J}^{k} \in \mathbb{F}_{2}$, such that

$$
\mathrm{Sq}^{k} s_{I}=\sum c_{I J}^{k} s_{J}, \quad \mathrm{Sq}^{k}\left[\sigma_{I^{m}}\right]=\sum c_{I J}^{k}\left[\sigma_{J^{m}}\right]
$$

holds for all $m$. Indeed, there are only finitely many $s_{I}$ in $H^{*}\left(\mathrm{Fl}_{\mathcal{D}^{\infty}} ; \mathbb{F}_{2}\right)$ of fixed codimension, and Steenrod operations are natural.

\subsubsection{Bockstein cohomology of even flag manifolds}

Now we start computing Bockstein cohomology using Proposition 6.3. Let

$$
Z_{m}:=\operatorname{ker}\left(\mathrm{Sq}^{1}: H^{*}\left(\mathrm{Fl}_{\mathcal{D}^{m}}\right) \rightarrow H^{*+1}\right), \quad B_{m}:=\operatorname{Im}\left(\mathrm{Sq}^{1}: H^{*-1}\left(\mathrm{Fl}_{\mathcal{D}^{m}}\right) \rightarrow H^{*}\right)
$$

both graded $\mathbb{F}_{2}$-vector spaces, let $H_{m}:=Z_{m} / B_{m}, q_{m}: Z_{m} \rightarrow H_{m}$ and denote $Z:=Z_{0}$ and $B:=B_{0}, H:=H_{0}, q:=q_{0}$. In the following we compare $Z_{m}$ with $Z$ and $B_{m}$ with $B$ using the description (6.5). Naturality of $\mathrm{Sq}^{1}$ implies that

(i) $B=\mathcal{K}_{m}^{*} B_{m}$,

(ii) $Z \supseteq \mathcal{K}_{m}^{*} Z_{m}$. 
These imply that $q \circ \mathcal{K}_{m}^{*}: Z_{m} \rightarrow Z \rightarrow H$ induces a map $\hat{\mathcal{K}}_{m}^{*}: H_{m} \rightarrow H$ satisfying

$$
\operatorname{ker} \hat{\mathcal{K}}_{m}^{*}=q_{m}\left(\operatorname{ker} \mathcal{K}_{m}^{*}\right)
$$

Lemma 6.12 Let $\mathcal{D}=\left(2 d_{1}, \ldots, 2 d_{r}\right)$. For even flag manifolds $\mathrm{Fl}_{\mathcal{D}}:$ If $\left[\sigma_{I}\right] \in Z$, then $\left[\sigma_{I}\right] \in \mathcal{K}_{1}^{*} Z_{1}$.

Proof Assume $\left[\sigma_{I}\right] \in Z$ for some $I \in \operatorname{OSP}(\mathcal{D})$. Write

$$
\mathrm{Sq}^{1}\left[\sigma_{I^{1}}\right]=\sum_{J \in T}\left[\sigma_{J}\right] \in H^{*}\left(\mathrm{Fl}_{\mathcal{D}^{1}}\right)
$$

where by assumption $\left[\sigma_{J}\right] \in \operatorname{ker} \mathcal{K}_{1}^{*}$ for all $J \in T \subseteq \operatorname{OSP}\left(\mathcal{D}^{1}\right)$. If $\left[\sigma_{J}\right] \in \operatorname{ker} \mathcal{K}_{1}^{*}$, then by (6.5) $J$ is obtained from $I^{1}$ by exchanging $\bar{N}+1 \in I_{r}^{1}$ with some $a \in I_{\alpha}^{1}$ which satisfies

$$
a=\max I_{\alpha}^{1}>\max I_{\alpha+1}^{1}>\ldots>\max I_{r-1}^{1}
$$

otherwise the number of inversions changes by at least 2 . To determine $\left[\Omega_{I}, \Omega_{J}\right]$, we use (6.3), recall the notations of (3.5). Clearly, $G_{I^{1}}(N+1, \alpha-1, r-1)=0$. By (6.7),

$$
L_{I^{1}}(a, \alpha, r)=\sum_{i=\alpha+1}^{r-1} 2 d_{i}
$$

so by (6.3), $\left[\Omega_{I}, \Omega_{J}\right]=0$. Hence the Steenrod coefficients also vanish by Proposition 6.3 , therefore $T$ is empty, proving the lemma.

Corollary 6.13 For even flag manifolds $\mathrm{Fl}_{2 \mathcal{D}}$ :

$$
Z=\mathcal{K}_{\infty}^{*} Z_{\infty}, \quad H=\hat{\mathcal{K}}_{\infty}^{*} H_{\infty},
$$

Proof Let $I \in \operatorname{OSP}(\mathcal{D})$ and $J \in \operatorname{OSP}\left(\mathcal{D}^{m}\right)$. A simple computation involving the number of inversions shows that $I^{m}$ and $J$ are adjacent in $\mathrm{Fl}_{\mathcal{D}^{m}}$ iff $J=K^{m-1}$ for some $K \in \operatorname{OSP}\left(\mathcal{D}^{1}\right)$ and $I^{1}$ and $K$ are adjacent in $\mathrm{Fl}_{\mathcal{D}^{1}}$. Therefore $\left[\sigma_{I^{1}}\right] \in Z_{1}$ iff $\left[\sigma_{I^{m}}\right] \in Z_{m}$, so $Z=\mathcal{K}_{1}^{*} Z_{1}=\mathcal{K}_{m}^{*} Z_{m}$ for all $m$. This also implies surjectivity of $\hat{\mathcal{K}}_{\infty}^{*}$

Now we can finally prove Theorem 6.1:

Proof of Theorem 6.1 It follows from results of Borel's thesis that $\operatorname{Tor}\left(H^{*}\left(\mathrm{Fl}_{\mathcal{D}} ; \mathbb{Z}\right)\right)$ is 2-primary (see [5, Propositions 29.1, 30.1]), so we can concentrate on $p=2$. By Corollary 6.13:

$$
P_{\beta}\left(\mathrm{Fl}_{2 \mathcal{D}}\right)=P_{\beta}\left(\mathrm{Fl}_{2 \mathcal{D}} \infty\right)-P\left(\operatorname{ker} \hat{\mathcal{K}}_{m}^{*}\right)
$$


Let $Q \subseteq Z_{m}$ be a subspace such that $q: Q \stackrel{\cong}{\rightarrow} H_{m}$. Then the Poincaré polynomials satisfy

$$
P\left(\operatorname{ker} \hat{\mathcal{K}}_{m}^{*}\right)=P\left(Q \cap \operatorname{ker} \mathcal{K}_{m}^{*}\right)
$$

By Proposition 6.10, such a subspace is given by $Q=\mathbb{F}_{2}\left[w_{2 j}\left(D_{i}\right)^{2}\right]$, so by (6.4)

$$
Q \cap \operatorname{ker} \mathcal{K}_{\infty}^{*}=\left\langle s_{J}\left(w_{2 j}^{2}\right): J \neq I^{\infty}, I \in \operatorname{OSP}(2 \mathcal{D})\right\rangle
$$

Denote the rational coefficient pullback induced by $\mathcal{K}_{\infty}$ by

$$
\mathcal{K}_{0}^{*}: H^{*}\left(\mathrm{Fl}_{2 \mathcal{D}} \infty ; \mathbb{Q}\right) \rightarrow H^{*}\left(\mathrm{Fl}_{2 \mathcal{D}} ; \mathbb{Q}\right)
$$

Since $\mathcal{K}_{0}^{*}$ is also surjective,

$$
P_{0}\left(\mathrm{Fl}_{2 \mathcal{D}}\right)=P_{0}\left(\mathrm{Fl}_{2 \mathcal{D}} \infty\right)-P\left(\operatorname{ker} \mathcal{K}_{0}^{*}\right)
$$

and

$$
\operatorname{ker} \mathcal{K}_{0}^{*}=\left\langle s_{J}\left(p_{j}\right): J \neq I^{\infty}, I \in \operatorname{OSP}(2 \mathcal{D})\right\rangle .
$$

Comparing (6.8) and (6.9), $P\left(\operatorname{ker} \mathcal{K}_{0}^{*}\right)=P\left(\operatorname{ker} \hat{\mathcal{K}}_{m}^{*}\right)$, and since $P_{0}\left(\mathrm{Fl}_{2 \mathcal{D}^{\infty}}\right)=$ $P_{\beta}\left(\mathrm{Fl}_{2 \mathcal{D}^{\infty}}\right)$ by Proposition $6.10, P_{0}\left(\mathrm{Fl}_{2 \mathcal{D}}\right)=P_{\beta}\left(\mathrm{Fl}_{2 \mathcal{D}}\right)$ and we can conclude by Proposition 6.9.

Remark 6.14 In the case of Grassmannians $\operatorname{Gr}_{K}\left(\mathbb{R}^{N}\right)$, the previous proof can also be adapted, and one can show that $P_{\beta}=P_{0}$. Lemma 6.12 also holds, except when $K$ is odd and $N$ even, when $Z \backslash Z_{1}$ also contains the $L$-Schubert classes. In the case of arbitrary flag manifolds $\mathrm{Fl}_{\mathcal{D}}$ these computations appear to be more complicated; it is no longer true that the Bockstein cohomology generators are Schubert varieties $\left[\sigma_{I}\right]$, but sums of them, see Appendix B.

Conjecture 6.15 For any real flag manifold (more generally any $R$-space) all torsion of $H^{*}\left(\mathrm{Fl}_{\mathcal{D}} ; \mathbb{Z}\right)$ is of order 2 .

Remark 6.16 If this conjecture holds, then the cohomology groups $H^{*}\left(\mathrm{Fl}_{\mathcal{D}}^{\mathbb{R}} ; \mathbb{Z}\right)$ can be determined from the Universal Coefficient Theorem as in [31], see also Proposition 6.2 .

\section{Enumerative applications: lower bounds}

The cohomology ring structure in terms of Schubert classes gives information about enumerative geometric Schubert problems: 
Given generic complete flags $F_{\bullet}^{1}, \ldots, F_{\bullet}^{r}$ in $\mathbb{R}^{N}$, what is the cardinality of

$$
\left|\bigcap_{j=1}^{r} \sigma_{\lambda_{j}}\left(F_{\bullet}^{j}\right)\right|=?
$$

for $\sigma_{j}:=\sigma_{\lambda_{j}}$ of total dimension $\operatorname{Gr}_{2 k}\left(\mathbb{R}^{2 n}\right)$ ?

The word generic is a subtle point here: we will say that the flags are generic, if the corresponding Schubert varieties are transversal. This is an open condition in the configuration space by the Kleiman-Bertini theorem. The main property of generic configurations $\mathcal{G}$ relevant to us is that the number of solutions is locally constant on $\mathcal{G}$.

In the complex case $\mathbb{F}=\mathbb{C}$, a Schubert problem can be solved by multiplying Schubert cycles: since everything is complex, at a smooth transversal intersection all tangent spaces have canonical orientations, therefore all intersections come with the same sign. Therefore the cohomology product of $\left[\sigma_{j}\right]$ is an element $n[*]$ of $H^{\text {top }}\left(\mathrm{Gr}_{k}\left(\mathbb{C}^{n}\right)\right) \cong \mathbb{Z}\langle[*]\rangle$, and this number $n$ is the answer to the Schubert problem.

In the real case $\mathbb{F}=\mathbb{R}$, there are no canonical orientations, therefore each transversal intersection $p$ comes with a sign, depending on whether the orientation of the tangent spaces $T_{p} \sigma_{j}$ agrees with the orientation of the tangent space $T_{p} \mathrm{Gr}_{k}\left(\mathbb{R}^{n}\right)$. Therefore the cohomological calculation only gives a signed sum of the points, hence a lower bound to the Schubert problem. The actual number of solutions depends on the configuration (the choice of the flags $F_{\bullet}^{i}$ ), and there is a range of numbers that might appear as the number of solutions. This range is not known in general, and it has been subject to extensive examination, see [34,48,49]. An infinite series of examples has been computed via elementary methods in the case of Grassmannians in [18]. For example, in $\mathrm{Gr}_{8}\left(\mathbb{R}^{16}\right)$ the number of solutions to the Schubert problem $\sigma_{\lambda}^{4}$ for $\lambda=(4,4,4,4)$ can be $\{6,14,30,70\}$, see [18]. Recently, similar problems were considered for Grassmannians using Chow-Witt rings [57].

The dependence of the number of solutions on the given configuration has the following explanation. In the complex case, the singular configurations form an at least one complex codimensional subvariety of the configuration space, so the space of nonsingular configurations is connected. In the real case, the singular configurations can be one real codimensional, in which case the configuration space falls apart into connected components (chambers).

An upper bound for the range is given by the number of solutions for the corresponding generic complex Schubert problem. Here some caution is required when discussing genericity: one has to show that there exist real generic flags which are complex generic when regarded as complex flags. Indeed, this is the case: the subset of complex nongeneric configurations can be defined by real equations, so there exist real flags $F_{\bullet}^{j}$ which are complex generic. For such flags, all intersections of $\sigma_{j}^{\mathbb{C}}\left(F_{\bullet}^{j}\right)$ are transverse, therefore so are those of $\sigma_{j}^{\mathbb{R}}\left(F_{\bullet}^{j}\right)$, so such configurations are also real generic.

It is a natural question, whether a real enumerative problem is maximal/fully real [49], i.e. whether there exists a configuration for which the number of solutions agrees 
with the number of solutions for the same complex problem. This is true for real Schubert problems in Grassmannians as shown in [53].

Another natural question in real enumerative geometry, is to find a lower bound for the range of solutions $[33,56]$. For example, by a Theorem of Segre, there are at least 3 lines on a cubic surface [43], more generally there are at least $(2 n-1)$ !! real lines on a degree $2 n-1$ hypersurface in $\mathbb{R} P^{n+1}$ [21]. For a lower bound on the number of 3-planes on an appropriate degree hypersurface see [20, Theorem 4.1.3]. See [39] for examples of enumerative problems with a strict lower bound of zero, i.e. no real solutions. As we have already mentioned, for Schubert calculus the cohomology calculation also gives a lower bound. By the description of the real Littlewood-Richardson coefficients (Corollary 4.5) we have:

Proposition 7.1 The number of solutions of a double real Schubert problem $\left(D \lambda_{j}\right)$ is bounded below by the number of solutions to the half sized complex one $\left(\lambda_{j}\right)$.

This means that any complex Schubert problem can be "doubled". We illustrate this via three examples.

Example 7.2 How many real 6-planes in $\mathbb{R}^{12}$ intersect 9 given 6-planes in at least 2 dimensions?

This problem can be written as $\square^{9}$ in $\mathrm{Gr}_{6}\left(\mathbb{R}^{12}\right)$. By the previous Proposition, a lower bound is given by the half-sized complex problem, namely $\square^{9}$ in $\mathrm{Gr}_{3}\left(\mathbb{C}^{6}\right)$. This in turn is equal to the degree of $\mathrm{Gr}_{3}\left(\mathbb{C}^{6}\right)$ via the Plücker embedding, which is 42 ; this is known in general, see e.g. [28, p. 247]. So the answer is 42 : there are at least 42 such real 6-planes.

Example 7.3 How many real 8-planes in $\mathbb{R}^{14}$ intersect 6 given 8 -planes in at least 4 dimensions?

This problem is

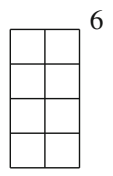

in $\mathrm{Gr}_{8}\left(\mathbb{R}^{14}\right)$. Again, a lower bound is given by the half-sized complex problem, namely $\square^{6}$ in $\mathrm{Gr}_{4}\left(\mathbb{C}^{7}\right)$. A computation using the Pieri rule and duality shows that the answer to the complex problem is $\square^{6}=16$. So the real problem has at least 16 solutions.

Example 7.4 We also give an example which uses the product structure on flag manifolds. Given the following ten generic subspaces in $\mathbb{C}^{5}$

$$
E_{1}^{i}, E_{3}^{i}, \quad i=1,2, \quad \text { and } E_{2}^{j}, E_{4}^{j}, \quad j=1,2,3,
$$

of $\operatorname{dimension} \operatorname{dim}\left(E_{i}^{j}\right)=i$, how many complete flags $F_{\bullet}$ in $\mathbb{C}^{5}$ satisfy the following conditions:

- $E_{1}^{i} \subseteq F_{4}$ and $\operatorname{dim} F_{2} \cap E_{3}^{i}=1$ for $i=1,2$,

- $F_{1} \subseteq E_{4}^{j}$ and $\operatorname{dim} F_{3} \cap E_{2}^{j}=1$ for $j=1,2,3$.

This corresponds to the product of special Schubert classes in $H^{*}\left(\mathrm{Fl}\left(\mathbb{C}^{5}\right)\right)$

$$
[\sigma(4)]^{2}[\sigma(2)]^{2}[\sigma(1)]^{3}[\sigma(3)]^{3}=7
$$


where we denote by $\sigma(i)$ the one codimensional Schubert variety corresponding to the transposition $(i, i+1)$. This problem then can be doubled: Given the following generic subspaces in $\mathbb{R}^{10}$

$$
E_{1}^{i}, E_{3}^{i}, \quad i=1,2, \quad \text { and } E_{2}^{j}, E_{4}^{j}, \quad j=1,2,3
$$

of dimension $\operatorname{dim}\left(E_{i}^{j}\right)=2 i$, how many flags $F_{\bullet}$ in $\mathrm{Fl}_{\mathcal{D}}\left(\mathbb{R}^{10}\right), \mathcal{D}=(2,2,2,2,2)$ satisfy the following conditions:

- $E_{1}^{i} \subseteq F_{4}$ and $\operatorname{dim} F_{2} \cap E_{3}^{i}=2$ for $i=1,2$,

- $F_{1} \subseteq E_{4}^{j}$ and $\operatorname{dim} F_{3} \cap E_{2}^{j}=2$ for $j=1,2,3$.

Using Proposition 7.1, the complex answer implies that this problem has at least 7 solutions. $\mathrm{Fl}\left(\mathbb{C}^{5}\right)$ was the smallest flag variety that we found with a nontrivial Schubert problem that cannot be reduced to the case of Grassmannians.

As we have seen, the cohomology of real Grassmannians (and even flag manifolds) with integer coefficients contains only 2 -torsion. If in a Schubert problem all Schubert varieties are cycles, but one of them is $\mathbb{Z}_{2}$-torsion, then the corresponding cycles multiply to zero in cohomology (at least if the flag manifold is orientable), which is a trivial lower bound. Note however that the corresponding Schubert problem can be, and usually is nontrivial. There exist enumerative problems, which do not have any cohomological interpretation: the corresponding Schubert varieties are not cycles. For example, dual transversal Schubert varieties always intersect in one point, and not all Schubert varieties are cycles. Summarizing: for the purpose of obtaining enumerative lower bounds, we don't lose anything by working with rational coefficient cohomology.

Alternatively, considering $\mathbb{F}_{2}$-coefficient cohomology, one can apply the original Borel-Haefliger theorem [7] to Grassmannians to obtain mod 2 information about a Schubert problem:

Proposition 7.5 The number of solutions of a real Schubert problem has the same parity as the number of solutions of the corresponding complex Schubert problem.

For certain Schubert problems, one can say more than mod 2 congruence of the solutions, see e.g. [18, Theorem 5.7] and [34]. We conclude with a conjecture:

Conjecture 7.6 The lower bound of Proposition 7.1 is sharp.

We make some remarks on this conjecture. Take real complete flags $F_{\bullet}^{j} \in \operatorname{Fl}\left(\mathbb{R}^{n}\right)$, such that $F_{2 i}^{j}$ are $\mathrm{U}(1)$-invariant. Then the set of solutions

$$
S:=\bigcap_{j} \sigma_{D I_{j}}^{\mathbb{R}}\left(F_{\bullet}^{j}\right)
$$

is a $\mathrm{U}(1)$-invariant subset. If $S$ is finite, then each point $W \in S$ is a $\mathrm{U}(1)$-fixed point, i.e. complex. Therefore, each $W \in S$ is a solution to the corresponding half sized complex Schubert problem

$$
W \in S_{\mathbb{C}}=\bigcap_{j} \sigma_{I_{j}}^{\mathbb{C}}\left(F_{\bullet, \mathbb{C}}^{j}\right),
$$


so this problem has exactly as many solutions as the half sized complex Schubert problem. However, it is not clear to us whether such a problem is real generic, which is required in order to show that this is a sharp lower bound. This reduces the conjecture to one about genericity: Does there exist a complex generic configuration of flags $F_{\bullet, \mathbb{C}}^{j}$, which as real flags $F_{\bullet, \mathbb{R}}^{j}$ are real generic (for the double sized real problem)?

Acknowledgements This paper is based on the author's $\mathrm{PhD}$ thesis [41]. I am grateful to my $\mathrm{PhD}$ supervisor László M. Fehér for many valuable discussions and helpful comments that improved the quality of this paper. I thank Liviu Mare for several discussions on the topics of this paper. I am grateful to the anonymous referees for carefully reading the manuscript and for their suggestions, which greatly improved the presentation of the paper.

Funding. Open access funding provided by ELKH Alfréd Rényi Institute of Mathematics. This research was partially supported by the Hungarian National Research, Development and Innovation Office, NKFIH K 119934.

Data availability. Data sharing not applicable to this article as no datasets were generated or analysed during the current study.

\section{Declarations}

Conflict of interest. On behalf of all authors, the corresponding author states that there is no conflict of interest.

Open Access This article is licensed under a Creative Commons Attribution 4.0 International License, which permits use, sharing, adaptation, distribution and reproduction in any medium or format, as long as you give appropriate credit to the original author(s) and the source, provide a link to the Creative Commons licence, and indicate if changes were made. The images or other third party material in this article are included in the article's Creative Commons licence, unless indicated otherwise in a credit line to the material. If material is not included in the article's Creative Commons licence and your intended use is not permitted by statutory regulation or exceeds the permitted use, you will need to obtain permission directly from the copyright holder. To view a copy of this licence, visit http://creativecommons.org/licenses/by/4.0/.

\section{Appendix A. Topology: Cartan model}

A pair $(G, K)$ of compact connected Lie groups is a Cartan pair, if $K \subseteq G$ is a subgroup and $H_{G}^{*}$ is a polynomial ring in rk $G$ many generators, rk $G-\operatorname{rk} K$ many of which restrict to zero via $\rho^{*}: H_{G}^{*} \rightarrow H_{K}^{*}$.

For Cartan pairs, there is a simple description of $H^{*}(G / K ; \mathbb{Q})$, due to Cartan and Borel [5], see also [51] for a summary.

Theorem A.1 (Borel, Cartan) For a Cartan pair $(G, K)$

$$
H^{*}(G / K ; \mathbb{Q}) \cong H_{K}^{*} /\left(\operatorname{Im} \rho^{*}\right)_{+} \bigotimes \bigwedge\left[x_{r_{i}-1}\right]_{i=p+1}^{n}
$$

where $r_{i}$ are the degrees of the polynomial generators restricting to zero via $\rho^{*}$ : $H_{G}^{*} \rightarrow H_{K}^{*}, n=\operatorname{rk} G, p=\operatorname{rk} K, \operatorname{deg} x_{j}=j$ and $(W)_{+}$denotes the ideal generated by elements of positive degree of the subspace $W$. 
Let $\mathrm{SO}(\mathcal{D}):=\prod_{i=1}^{m} \mathrm{SO}\left(d_{i}\right), \mathrm{O}(\mathcal{D}):=\prod_{i=1}^{m} \mathrm{O}\left(d_{i}\right) N:=\sum d_{i}$.

Proposition A.2 $\left(G, K_{0}\right)=(\mathrm{SO}(N), \mathrm{SO}(\mathcal{D}))$ is a Cartan pair for all $\mathcal{D}$.

Proof One has

$$
\rho^{*}\left(p_{*}(S)\right)=\prod_{i=1}^{m} p_{*}\left(S_{i}\right),
$$

where $S \rightarrow B S O(N)$ and $S_{i} \rightarrow B S O\left(d_{i}\right)$ denote the tautological bundles and $p_{*}$ denotes the total Pontryagin class. Let $n=\left\lfloor\frac{N}{2}\right\rfloor$ be the rank of $\operatorname{SO}(N)$ and $q=$ $\sum_{i=1}^{m}\left\lfloor\frac{d_{i}}{2}\right\rfloor$ be the rank of $\operatorname{SO}(\mathcal{D})$. Since $p_{\text {top }}\left(S_{i}\right)=p_{\left\lfloor\frac{d_{i}}{2}\right\rfloor}\left(S_{i}\right)$, by examining degrees, one sees that $\rho^{*}\left(p_{j}(S)\right)=0$ for $j>q$.

The real flag manifold is a homogeneous space $\mathrm{Fl}_{\mathcal{D}}^{\mathbb{R}}=G / K$, where $G=S O(N)$ and $K=S(\mathrm{O}(\mathcal{D}))$. Since $K$ is not connected, the Borel-Cartan model cannot be directly applied. $H^{*}(G / K ; \mathbb{Q})$ was recently determined by He in [32], we state the theorem, also to be found in [41].

Let $\mathcal{D}_{0}=\left(\left\lfloor d_{1} / 2\right\rfloor,\left\lfloor d_{2} / 2\right\rfloor, \ldots,\left\lfloor d_{m} / 2\right\rfloor\right)$. Since there is a covering $\Gamma \rightarrow G / K_{0} \rightarrow$ $G / K$ for $\Gamma=\mathbb{Z}_{2}^{m-1}, H^{*}(G / K)=\left(H^{*}\left(G / K_{0}\right)\right)^{\Gamma}$. The $\Gamma$-action acts by multiplying the Euler classes in $H_{K}^{*}$ by -1 and trivially on the Pontryagin classes and on the exterior algebra, for further details see [32] or [41].

Theorem A.3 Let $n=\left\lfloor\frac{N}{2}\right\rfloor$ be the rank of $\mathrm{SO}(N)$ and $q=\sum_{i=1}^{m}\left\lfloor\frac{d_{i}}{2}\right\rfloor$ be the rank of $\mathrm{SO}(\mathcal{D})$, then

$$
H^{*}\left(\mathrm{Fl}_{\mathcal{D}}^{\mathbb{R}} ; \mathbb{Q}\right) \cong H^{2 *}\left(\mathrm{Fl}_{\mathcal{D}_{0}}^{\mathbb{C}} ; \mathbb{Q}\right) \otimes \bigwedge\left[y_{i}\right]_{i=q+1}^{n}
$$

where $y_{i}=x_{4 i-1}$ except if $N$ even, $y_{n}=x_{N-1} . H^{2 *}$ means that the degrees are doubled and $\operatorname{deg} x_{j}=j$. The first term $H^{2 *}$ is generated as an algebra by $p_{i}\left(D_{j}\right)$ with the relations given by the identity $\prod_{j=1}^{m} p_{*}\left(D_{j}\right)=1$.

\section{Appendix B. Schubert cycles: the general case of partial flag manifolds}

Using the coefficients of the Vassiliev complex described in Section 3, we computed some of the generators of $H^{*}\left(\mathrm{Fl}_{\mathcal{D}}^{\mathbb{R}} ; \mathbb{Q}\right)$ in terms of Schubert cells using SageMath's homology package [52]. We include below some cases which are not covered by Theorems 3.12 and 5.1, in particular, complete, odd and other examples. As previously mentioned, for general $\mathrm{Fl}_{\mathcal{D}}$ it is no longer true that rational cohomology classes can be represented by Schubert varieties, but a signed sum of Schubert cells. The choice of the generators are not unique, as we already saw on the example of $\mathrm{Fl}\left(\mathbb{R}^{4}\right)$, Section 3.10. In all the examples we have computed, the coefficients of these Schubert cells are \pm 1 . In the tables, we use the following conventions.

Notation. The Schubert cells $\Omega_{I} \subseteq \mathrm{Fl}_{\mathcal{D}}$ are parametrized by ordered set partitions $I \in \operatorname{OSP}(\mathcal{D})$. These are denoted by the one-line notation of their minimal representatives: elements of $I_{j}$ are listed in increasing order, $I_{j}$ and $I_{j+1}$ is separated by a 
comma. The + sign separates the Schubert cells whose sums are generators. We do not keep track of the sign of the cells, as this can vary according to convention (even though relative to each other, the signs do make sense). In the last table, the ordered set partitions are elements of $1,2, \ldots, 11$; for typographical reasons 10 and 11 are preceded by a space.

\section{B. 1. The complete case}

The two extreme cases of real flag manifolds are Grassmannians and complete flag manifolds. We understand the Schubert calculus of Grassmannians by Propositions 5.2 and 5.3. For the case of complete flag manifolds, the answer appears to be less simple, as we illustrate in Tables 2 and 3. For the case of $\mathrm{Fl}\left(\mathbb{R}^{3}\right)$ see also [38, p. 5] and for $\mathrm{Fl}\left(\mathbb{R}^{4}\right)$, see also [12, p. 529].

Table 2 Sums of Schubert cells generating $H^{*}\left(\mathrm{Fl}\left(\mathbb{R}^{n}\right) ; \mathbb{Q}\right)$, labeled by permutations $S_{n}$

\begin{tabular}{lllll}
\hline $\operatorname{deg}$ & $\begin{array}{lll}\mathrm{Fl}\left(\mathbb{R}^{3}\right) \\
\Lambda\left[x_{3}\right]\end{array}$ & $\begin{array}{l}\mathrm{Fl}\left(\mathbb{R}^{4}\right) \\
\Lambda\left[x_{3}, y_{3}\right]\end{array}$ & $\begin{array}{l}\mathrm{Fl}\left(\mathbb{R}^{5}\right) \\
\Lambda\left[x_{3}, x_{7}\right]\end{array}$ & $\begin{array}{l}\mathrm{Fl}\left(\mathbb{R}^{6}\right) \\
\Lambda\left[x_{3}, x_{7}, y_{5}\right]\end{array}$ \\
\hline 0 & 321 & 4321 & 54321 & 654321 \\
3 & 123 & $2341+4123$ & $34521+52341+54123$ & $456321+634521+652341+654123$ \\
3 & 3214 & & \\
5 & & & 365214 \\
6 & 1234 & 14325 & \\
7 & & & $432561+632145$ \\
8 & & 1234 & $345216+523416+541236$ \\
10 & & & $234561+236145+412563+612345$ \\
12 & & & 125436 \\
15 & & & 123456 \\
\hline
\end{tabular}

Table 3 Sums of Schubert cells generating $H^{*}\left(\mathrm{Fl}\left(\mathbb{R}^{7}\right) ; \mathbb{Q}\right)$, labeled by permutations $S_{7}$

\begin{tabular}{ll}
\hline deg & $\begin{array}{l}\mathrm{Fl}\left(\mathbb{R}^{7}\right) \\
\Lambda\left[x_{3}, x_{7}, x_{11}\right]\end{array}$ \\
\hline 0 & 7654321 \\
3 & $5674321+7456321+7634521+7652341+7654123$ \\
7 & $5436721+7432561+7632145$ \\
10 & $3456721+3472561+3672145+5236741+5416723+5436127+$ \\
& $+5632147+7234561+7236145+7412563+7612345$ \\
11 & 1476325 \\
14 & $1456327+3416527+5216347+5412367$ \\
18 & 1236547 \\
21 & 1234567 \\
\hline
\end{tabular}


Table 4 Sums of Schubert cells generating $H^{*}\left(\mathrm{Fl}_{333} ; \mathbb{Q}\right)$, labeled by $\operatorname{OSP}(3,3,3)$

\begin{tabular}{ll}
\hline deg & $\begin{array}{l}\mathrm{Fl}_{333} \\
H^{*}\left(\mathrm{Fl}_{222} ; \mathbb{Q}\right) \otimes \Lambda\left[x_{15}\right]\end{array}$ \\
\hline 0 & $789,456,123$ \\
4 & $789,236,145$ \\
4 & $569,478,123$ \\
8 & $349,678,125+369,458,127+389,256,147+589,234,167$ \\
8 & $569,238,147+589,234,167$ \\
12 & $349,258,167$ \\
15 & $167,258,349$ \\
19 & $167,234,589$ \\
19 & $145,278,369+147,256,389$ \\
23 & $123,478,569$ \\
23 & $145,236,789$ \\
27 & $123,456,789$ \\
\hline
\end{tabular}

\section{B. 2. The odd case}

There are two cases when flag manifolds $\mathrm{Fl}_{\mathcal{D}}^{\mathbb{R}}$ are orientable; all $d_{i}$ are even or all odd. If all $d_{i}$ are even, we understand the generators of rational cohomology by Theorem 3.12. If all $d_{i}$ are odd, the answer again appears to be less simple, see Tables 4 and 6 (and also the complete cases).

\section{B. 3. The other cases}

See Table 5 for a nonorientable case.

Table 5 Sums of Schubert cells generating $H^{*}\left(\mathrm{Fl}_{234} ; \mathbb{Q}\right)$, labeled by $\operatorname{OSP}(2,3,4)$

\begin{tabular}{ll}
\hline $\operatorname{deg}$ & $\mathrm{Fl}_{234}$ \\
& $H^{*}\left(\mathrm{Fl}_{224} ; \mathbb{Q}\right)$ \\
\hline 0 & $89,567,1234$ \\
4 & $89,347,1256$ \\
4 & $67,589,1234$ \\
8 & $89,127,3456$ \\
8 & $45,789,1236$ \\
8 & $47,569,1238+67,349,1258$ \\
12 & $45,369,1278$ \\
12 & $23,789,1456$ \\
12 & $27,369,1458+67,129,3458$ \\
16 & $23,569,1478$ \\
16 & $25,349,1678+45,129,3678$ \\
20 & $23,149,5678$ \\
\hline
\end{tabular}


Table 6 Sums of Schubert cells generating $H^{*}\left(\mathrm{Fl}_{335} ; \mathbb{Q}\right)$, labeled by $\operatorname{OSP}(3,3,5)$

\begin{tabular}{ll}
\hline deg & $\mathrm{Fl}_{335}$ \\
& $H^{*}\left(\mathrm{Fl}_{224} ; \mathbb{Q}\right) \otimes \Lambda\left[x_{19}\right]$ \\
\hline 0 & $91011,678,12345$ \\
4 & $91011,458,12367$ \\
4 & $7811,6910,12345$ \\
8 & $91011,238,14567$ \\
8 & $5611,8910,12347$ \\
8 & $5811,6710,12349+7811,4510,12369$ \\
12 & $5611,4710,12389$ \\
12 & $3411,8910,12567$ \\
12 & $3811,4710,12569+7811,2310,14569$ \\
16 & $3411,6710,12589$ \\
16 & $3611,4510,12789+5611,2310,14789$ \\
19 & $189,2710,345611$ \\
20 & $3411,2510,16789$ \\
23 & $127,8910,345611+167,2910,345811$ \\
23 & $169,2510,347811+189,256,3471011$ \\
27 & $167,258,3491011$ \\
27 & $125,6910,347811+145,2910,367811$ \\
27 & $149,2310,567811+189,234,5671011$ \\
31 & $125,678,3491011+145,278,3691011$ \\
31 & $123,4910,567811$ \\
31 & $147,238,5691011+167,234,5891011$ \\
35 & $145,236,7891011$ \\
35 & $123,478,5691011$ \\
39 & $123,456,7891011$ \\
\hline & \\
\hline & \\
\hline & \\
\hline &
\end{tabular}

These examples hopefully illustrate that although there is a simple description of the cohomology of real flag manifolds in terms of topology (cf. Cartan model, Appendix A), in general there is some nontrivial combinatorics involved in translating that description to the Schubert calculus setting.

\section{References}

1. Allday, C., Puppe, V.: Cohomological methods in transformation groups. Cambridge University Press, Cambridge (2007)

2. Arnold, V.I., Vassiliev, V.A., Goryunov, V.V., Lyashko, O.V.: Singularity theory I. Dynamical systems VI, VINITI (1988)

3. Bernstein, I.N., Gel'fand, I.M., Gel'fand, S.I.: Schubert cells, and the cohomology of the spaces $G / P$. Uspehi Mat. Nauk 28(3(171)), 3-26 (1973)

4. Borel, A., Hirzebruch, F.: Characteristic classes and homogeneous spaces. II. Am. J. Math. 81, 315-382 (1959)

5. Borel, A.: Sur la cohomologie des espaces fibrés principaux et des espaces homogènes de groupes de Lie compacts. Ann. Math. 2(57), 115-207 (1953) 
6. Borel, A.: Topics in the homology theory of fibre bundles. Lectures given at the University of Chicago, 1954. Springer-Verlag, Berlin (1967)

7. Borel, A., Haefliger, André: La classe d'homologie fondamentale d'un espace analytique. Bulletin de la Société Mathématique de France 89, 461-513 (1961)

8. Bott, R., Tu, LW.: Differential forms in algebraic topology. Graduate Texts in Mathematics, vol. 82. Springer-Verlag, New York-Berlin (1982)

9. Michel B.: Lectures on the geometry of flag varieties. In Topics in cohomological studies of algebraic varieties, pages 33-85. Birkhäuser Basel (2005)

10. Carlson, Jeffrey D.: The Borel equivariant cohomology of real Grassmannians. arXiv:1611.01175 (2016)

11. Casian, L., Kodama, Y.: On the cohomology of real Grassmann manifolds. arXiv:1309.5520 (2013)

12. Casian, L., Stanton, RJ.: Schubert cells and representation theory. Invent. Math. 137(3), 461-539 (1999)

13. Chern, Shiing-Shen.: Topics in differential geometry. The Institute for Advanced Study (1951)

14. Duan, H., Zhao, X.: A unified formula for Steenrod operations in flag manifolds. Compos. Math. 143(1), 257-270 (2007)

15. Duistermaat, J.J., Kolk, J.A.C., Varadarajan, V.S.: Functions, flows and oscillatory integrals on flag manifolds and conjugacy classes in real semisimple Lie groups. Compos. Math. 49(3), 309-398 (1983)

16. Ehresmann, C.: Sur la topologie de certaines variétés algébriques réelles. J. Math. Pures Appl 16(9), 69-100 (1937)

17. Fehér, László M.,Matszangosz, Ákos K. : Halving spaces and lower bounds in real enumerative geometry. arXiv:2006.11251

18. Fehér, László. M., Matszangosz, Ákos. K.: Real solutions of a problem in enumerative geometry. Period. Math. Hungar. 73(2), 137-156 (2016)

19. Fehér, László. M., Rimányi, Richárd: Thom polynomials with integer coefficients. Illin. J. Math. 46(4), 1145-1158 (2002)

20. Finashin, S., Kharlamov, V.: Abundance of 3-planes on real projective hypersurfaces. Arnold Math. J. 1(2), 171-199 (2015)

21. Finashin, S., Kharlamov, V.: Abundance of real lines on real projective hypersurfaces. Int. Math. Res. Not. IMRN 16, 3639-3646 (2013)

22. Franz, M., Puppe, V.: Steenrod squares on conjugation spaces. Comptes Rendus Mathematique 342(3), 187-190 (2006)

23. Fuchs, D. B.: Classical manifolds. In Topology. II, volume 24 of Encyclopaedia Math. Sci., pages 197-252. Springer, Berlin. Translated from the Russian by the author (2004)

24. Fulton, W.: Flags, Schubert polynomials, degeneracy loci, and determinantal formulas. Duke Math. J. 65(3), 381-420 (1992)

25. Fulton, W.: Young tableaux. London Mathematical Society Student Texts. Cambridge University Press, Cambridge (1997)

26. Fulton, W., Pragacz, P.: Schubert varieties and degeneracy loci. Lecture Notes in Mathematics, vol. 1689. Springer-Verlag, Berlin (1998)

27. Gorbatsevich, V. V., Onishchik, A. L.: Lie groups and Lie algebras, I: Lie transformation groups. Encyclopaedia of Mathematical Sciences, 20:95-235 Springer, Berlin (1993)

28. Harris, J.: Algebraic geometry, volume 133 of Graduate Texts in Mathematics. Springer-Verlag, New York, (1995). A first course, Corrected reprint of the 1992 original

29. Hausmann, Jean-Claude., Holm, Tara S., Puppe, Volker: Conjugation spaces. Algebraic \& Geometric Topology 5(3), 923-964 (2005)

30. He, Chen: Localization of equivariant cohomology rings of real Grassmannians. arXiv:1609.06243 (2016)

31. He, Chen: Torsions of integral homology and cohomology of real Grassmannians. arXiv:1709.05623 (2017)

32. He, C.: Cohomology rings of the real and oriented partial flag manifolds. Topol. Appl., 279:107239, 25, (2020)

33. Hein, N., Hillar, C.J., Sottile, F.: Lower bounds in real Schubert calculus. São Paulo J. Math. Sci. 7(1), 33-58 (2013)

34. Hein, N., Sottile, F., Zelenko, I.: A congruence modulo four in real Schubert calculus. J. Reine Angew. Math. 714, 151-174 (2016) 
35. Kirwan, FC.: Cohomology of quotients in symplectic and algebraic geometry. Mathematical Notes, vol. 31. Princeton University Press, Princeton, NJ (1984)

36. Kleiman, S. L., Laksov, D.: Schubert calculus. Am. Math. Month. 79(10), 1061-1082 (1972)

37. Knutson, A., Tao, T.: Puzzles and (equivariant) cohomology of Grassmannians. Duke Math. J. 119(2), 221-260 (2003)

38. Kocherlakota, R.R.: Integral homology of real flag manifolds and loop spaces of symmetric spaces. Adv. Math. 110, 1-46 (1995)

39. Kollár, János.: Examples of vanishing Gromov-Witten-Welschinger invariants. J. Math. Sci. Univ. Tokyo 22(1), 261-278 (2015)

40. Lenart, C.: The combinatorics of Steenrod operations on the cohomology of Grassmannians. Adv. Math. 136(2), 251-283 (1998)

41. Matszangosz, Ákos K.: Borel-Haefliger type theorems. PhD thesis, Central European University, (2019). http://www.etd.ceu.edu/2019/matszangosz_kyriakos.pdf

42. Mosher, Robert E., Tangora, Martin C.: Cohomology operations and applications in homotopy theory. Harper \& Row, Publishers, New York-London (1968)

43. Okonek, C., Teleman, A.: Intrinsic signs and lower bounds in real algebraic geometry. J. Reine Angew. Math. 688, 219-241 (2014)

44. Pontryagin, L.S.: Characteristic cycles on differentiable manifolds. Mat. Sbornik N. S. 21(63), 233-284 (1947)

45. Quillen, D.: Elementary proofs of some results of cobordism theory using Steenrod operations. Adv. Math. 7(1), 29-56 (1971)

46. Rabelo, L., San Martin, LAB.: Cellular homology of real flag manifolds. Indagationes Mathematicae, 30(5):745-772 (2019)

47. Sadykov, R.: Elementary calculation of the cohomology rings of real Grassmann manifolds. Pacific J. Math. 289(2), 443-447 (2017)

48. Soprunova, E., Sottile, F.: Lower bounds for real solutions to sparse polynomial systems. Adv. Math. 204(1), 116-151 (2006)

49. Sottile, F.: Enumerative geometry for the real Grassmannian of lines in projective space. Duke Math. J. 87(1), 59-85 (1997)

50. Takeuchi, M.: On Pontrjagin classes of compact symmetric spaces. J. Fac. Sci. Univ. Tokyo Sect. I(9), 313-328 (1962)

51. Terzić, Svjetlana: On real cohomology generators of compact homogeneous spaces. Sarajevo J. Math., 7(20)(2):277-287 (2011)

52. The Sage Developers. SageMath, the Sage Mathematics Software System (Version 8.1), (2017). https:// www.sagemath.org

53. Vakil, R.: Schubert induction. Ann Math. 164, 489-512 (2006)

54. Van Hamel, J.: Geometric cohomology frames on Hausmann-Holm-Puppe conjugation spaces. Proc. Am. Math. Soc. 135(5), 1557-1564 (2007)

55. Vassiliev, V.: Lagrange and Legendre Characteristic Classes, volume 3 of Advanced studies in contemporary mathematics. Taylor \& Francis, (1988)

56. Welschinger, J. Y.: Invariants of real symplectic 4-manifolds and lower bounds in real enumerative geometry. Invent. Math. 162(1), 195-234 (2005)

57. Wendt, M.: Oriented Schubert calculus in Chow-Witt rings of Grassmannians. Motivic homotopy theory and refined enumerative geometry, 217-267, Contemp. Math., 745, Amer. Math. Soc., Providence, RI, (2020)

58. Whitney, H.: Elementary structure of real algebraic varieties. Ann. Math. 2(66), 545-556 (1957)

Publisher's Note Springer Nature remains neutral with regard to jurisdictional claims in published maps and institutional affiliations. 